\title{
BEST POSSIBLE ESTIMATES OF SOLUTIONS TO THE TRANSMISSION PROBLEM FOR THE LAPLACE OPERATOR WITH $N$ DIFFERENT MEDIA IN A CONICAL DOMAIN
}

Abstract. We investigate the behavior of weak solutions to the transmission problem for the Laplace operator with $N$ different media in a neighborhood of a boundary conical point. We establish a precise exponent of the decreasing rate of the solution.

1. Introduction. Let $G \subset \mathbb{R}^{n}, n \geq 2$, be a bounded domain with boundary $\partial G$ that is a smooth surface everywhere except at the origin $\mathcal{O} \in \partial G$ and near $\mathcal{O}$ it is a convex conical surface with vertex at $\mathcal{O}$ and aperture $\omega_{0} \in$ $(0, \pi)$. We assume that $G=\bigcup_{i=1}^{N} G_{i}$ is divided into $N$ subdomains $G_{i}, i=$ $1, \ldots, N$, by $N-1$ hyperplanes $\Sigma_{k}, k=1, \ldots, N-1$, where $\mathcal{O}$ belongs to every $\bar{\Sigma}_{k}$ and $G_{i} \cap G_{j}=\emptyset, i \neq j$. Let $\omega_{i}$ be the aperture of $G_{i}$ at $\mathcal{O}$. Define $\theta_{k}=$ $\omega_{1}+\cdots+\omega_{k}$, thus $\omega_{0}=\theta_{N}$. Let $\mathcal{D} \subseteq \partial G$ be the part of $\partial G$ where we consider the Dirichlet boundary condition. We consider the transmission problem

$$
\left\{\begin{array}{r}
\mathcal{L}_{i}[u] \equiv a_{i} \Delta u_{i}-p_{i} u_{i}(x)=f_{i}(x), \quad x \in G_{i}, i=1, \ldots, N \\
{[u]_{\Sigma_{k}}=0, \quad \mathcal{S}_{k}[u] \equiv\left[a \frac{\partial u}{\partial n_{k}}\right]_{\Sigma_{k}}+\frac{1}{|x|} \beta_{k}(\phi) u(x)=h_{k}(x),} \\
x \in \Sigma_{k}, k=1, \ldots, N-1 ; \\
\mathcal{B}[u] \equiv \alpha(x) \cdot a \frac{\partial u}{\partial \vec{n}}+\frac{1}{|x|} \gamma(\phi) u(x)=g(x), \quad x \in \partial G \backslash \mathcal{O},
\end{array}\right.
$$

where $a_{i}>0, p_{i} \geq 0(i=1, \ldots, N)$ are constants;

$$
\alpha(x)= \begin{cases}0 & \text { if } x \in \mathcal{D} \\ 1 & \text { if } x \notin \mathcal{D}\end{cases}
$$

2000 Mathematics Subject Classification: 35J25, 35J60, 35J85, 35B65.

Key words and phrases: elliptic equations, transmission problem, conical points. 
$\vec{n}$ denotes the unit outward normal to $\partial G \backslash \mathcal{O}$; moreover

- $u(x)=u_{i}(x), x \in G_{i} ; f(x)=f_{i}(x), x \in G_{i} ;\left.a\right|_{\bar{G}_{i}}=a_{i}$, etc.;

- $[u]_{\Sigma_{k}}$ denotes the jump of $u$ across $\Sigma_{k}$, i.e.

$$
\begin{gathered}
{[u]_{\Sigma_{k}}=\left.u_{k}(\bar{x})\right|_{\Sigma_{k}}-\left.u_{k+1}(\bar{x})\right|_{\Sigma_{k}},\left.\quad u_{k}(\bar{x})\right|_{\Sigma_{k}}=\lim _{G_{k} \ni x \rightarrow \bar{x} \in \Sigma_{k}} u(x),} \\
\left.u_{k+1}(\bar{x})\right|_{\Sigma_{k}}=\lim _{G_{k+1} \ni x \rightarrow \bar{x} \in \Sigma_{k}} u(x) ;
\end{gathered}
$$

- $\left[a \frac{\partial u}{\partial \vec{n}_{k}}\right]_{\Sigma_{k}}$ denotes the jump of the co-normal derivative of $u$ across $\Sigma_{k}$, i.e.

$$
\left[a \frac{\partial u}{\partial \vec{n}_{k}}\right]_{\Sigma_{k}}=\left.a_{k} \frac{\partial u_{k}}{\partial \vec{n}_{k}}\right|_{\Sigma_{k}}-\left.a_{k+1} \frac{\partial u_{k+1}}{\partial \vec{n}_{k}}\right|_{\Sigma_{k}},
$$

where $\vec{n}_{k}$ denotes the unit outward normal to $\Sigma_{k}$.

The transmission problems often appear in different fields of physics and technique. For instance, an important problem in the electrodynamics of solid media is the electromagnetic processes in ferromagnetic media with different dielectric constants. These problems also appear in solid mechanics if a body consists of composite materials.

Here we obtain best possible estimates of weak solutions of problem $(L)$ near a conical boundary point. Analogous results were established in [3] for the Dirichlet and Robin problems in a conical domain without interfaces. Many mathematicians have considered the transmission problems. First, V. A. Il'in [9], O. A. Ladyzhenskaya and N. N. Ural'tseva [10], Z. G. Sheftel [13] and M. V. Borsuk [2] studied general interface problems for second order elliptic operators in smooth domains. Later other mathematicians studied transmission problems in nonsmooth domains in some particular cases (see the references in $[11,12,5]$ ). General interface problems in polygonal and polyhedral domains were considered in $[11,12]$. Regularity results in terms of weighted Sobolev spaces have been obtained in [5] for two- and three-dimensional transmission problems for the Laplace operator.

A principal new feature of this article is the derivation of sharp estimates for solutions of $(L)$ in $n$-dimensional $(n \geq 2)$ conic domains with $N$ different media $(N \geq 2)$. We show some examples. We also note that our methods are different from those in $[11,12]$.

We introduce the following notations:

- $S^{n-1}$ : the unit sphere in $\mathbb{R}^{n}$ centered at $\mathcal{O}$;

- $(r, \phi), \phi=\left(\phi_{1}, \ldots, \phi_{n-1}\right)$ : the spherical coordinates of $x \in \mathbb{R}^{n}$ with pole $\mathcal{O}$ : 


$$
\begin{aligned}
x_{1} & =r \cos \phi_{1}, \\
x_{2} & =r \cos \phi_{2} \sin \phi_{1}, \\
& \vdots \\
x_{n-1} & =r \cos \phi_{n-1} \sin \phi_{n-2} \ldots \sin \phi_{1}, \\
x_{n} & =r \sin \phi_{n-1} \sin \phi_{n-2} \ldots \sin \phi_{1} .
\end{aligned}
$$

- $\mathcal{C}$ : the convex rotational cone $\left\{x_{1}>r \cos \left(\omega_{0} / 2\right)\right\}$ with vertex $\mathcal{O}$;

- $\partial \mathcal{C}$ : the lateral surface of $\mathcal{C},\left\{x_{1}=r \cos \left(\omega_{0} / 2\right)\right\}$;

- $\Omega$ : a domain with smooth boundary $\partial \Omega$ obtained by intersecting the cone lateral surface $\partial \mathcal{C}$ with $S^{n-1}, \partial \Omega=\partial \mathcal{C} \cap S^{n-1}$;

- $\Omega_{i}$ : intersection of $G_{i}$ and $S^{n-1}(i=1, \ldots, N)$; thus $\Omega=\bigcup_{i=1}^{N} \Omega_{i}$;

- $G_{a}^{b}=\{(r, \omega) \mid 0 \leq a<r<b ; \omega \in \Omega\} \cap G$ : a layer in $\mathbb{R}^{n}$;

- $\Gamma_{a}^{b}=\{(r, \phi) \mid 0 \leq a<r<b ; \phi \in \partial \Omega\} \cap \partial G$ : the lateral surface of $G_{a}^{b}$;

- $G_{d}=G \backslash G_{0}^{d}, \Gamma_{d}=\partial G \backslash \Gamma_{0}^{d}, d>0$;

- $\Sigma=\sum_{k=1}^{N-1} \Sigma_{k}$, where $\Sigma_{k}=G \cap\left\{\phi_{1}=\omega_{0} / 2-\theta_{k}\right\}, k=1, \ldots, N-1$;

- $\sigma=\sum_{k=1}^{N-1} \sigma_{k}$, where $\sigma_{k}=\Sigma_{k} \cap \Omega$;

- $\Sigma_{a}^{b}=G_{a}^{b} \cap \Sigma ; \Sigma_{d}=\Sigma \backslash \Sigma_{0}^{d}, d>0$;

- $\left(G_{i}\right)_{a}^{b}=\{(r, \omega) \mid 0 \leq a<r<b ; \omega \in \Omega\} \cap G_{i}, i=1, \ldots, N$;

- $\left(\Sigma_{k}\right)_{a}^{b}=G_{a}^{b} \cap \Sigma_{k}, k=1, \ldots, N-1$;

- $\Omega_{\varrho}=G_{0}^{d} \cap\{|x|=\varrho\}, 0<\varrho<d$.

We assume without loss of generality that there exists $d>0$ such that $G_{0}^{d}$ is a convex rotational cone with vertex $\mathcal{O}$ and aperture $\omega_{0}$, thus

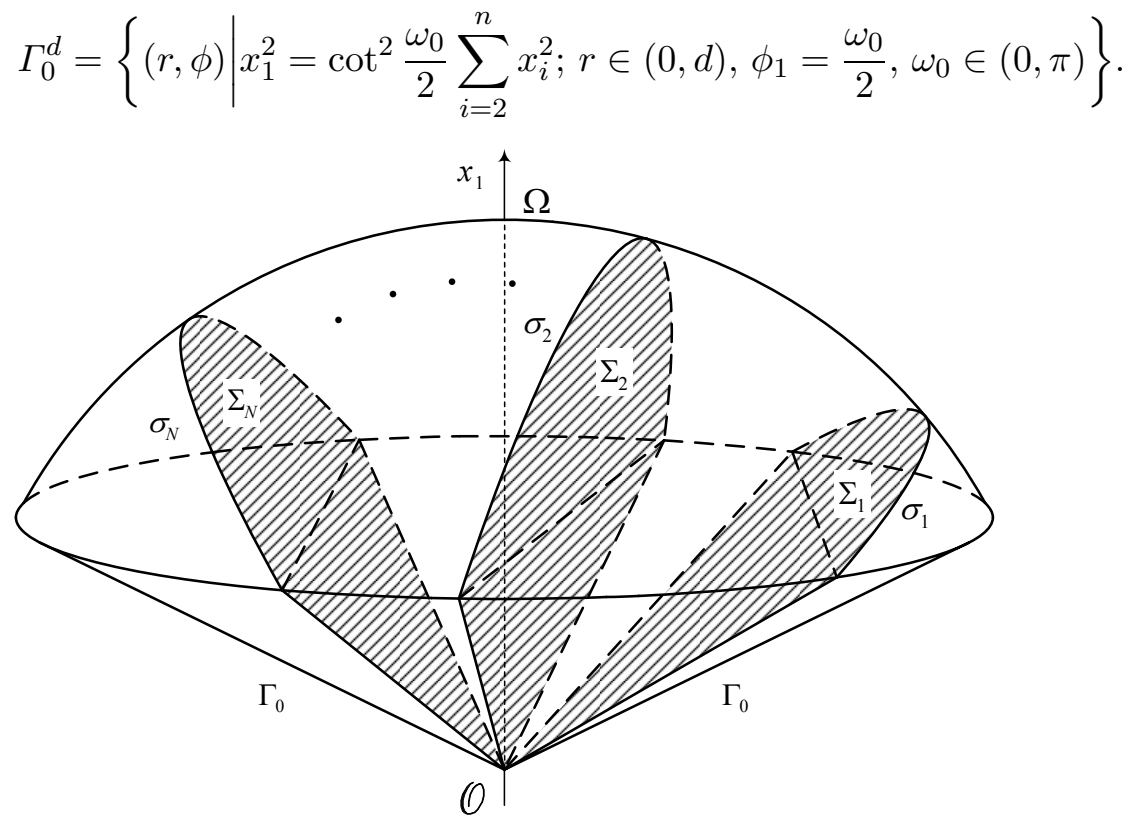

Fig. 1 
We use the standard function spaces: $C^{k}\left(\bar{G}_{i}\right)$ with the norm $\left|u_{i}\right|_{k, G_{i}}$; the Lebesgue space $L_{q}\left(G_{i}\right), q \geq 1$, with the norm $\left\|u_{i}\right\|_{q, G_{i}}$; the Sobolev space $W^{k, q}\left(G_{i}\right)$ with the norm $\left\|u_{i}\right\|_{k, q ; G_{i}}$; the direct sums $\mathbf{C}^{k}(\bar{G})=C^{k}\left(\bar{G}_{1}\right) \dot{+} \cdots \dot{+}$ $C^{k}\left(\bar{G}_{N}\right)$ with the norm $|u|_{k, G}=\sum_{i=1}^{N}\left|u_{i}\right|_{k, G_{i}} ; \mathbf{L}_{q}(G)=L_{q}\left(G_{1}\right) \dot{+} \cdots \dot{+} L_{q}\left(G_{N}\right)$ with the norm $\|u\|_{\mathbf{L}_{q}(G)}=\sum_{i=1}^{N}\left(\int_{G_{i}}\left|u_{i}\right|^{q} d x\right)^{1 / q} ; \mathbf{W}^{k, q}(G)=W^{k, q}\left(G_{1}\right) \dot{+} \cdots \dot{+}$ $W^{k, q}\left(G_{N}\right)$ with the norm $\|u\|_{k, q ; G}=\sum_{i=1}^{N}\left(\int_{G_{i}} \sum_{|\beta|=0}^{k}\left|D^{\beta} u_{i}\right|^{q} d x\right)^{1 / q}$. We define the weighted Sobolev spaces $\mathbf{V}_{q, \alpha}^{k}(G)=V_{q, \alpha}^{k}\left(G_{1}\right) \dot{+} \cdots \dot{+} V_{q, \alpha}^{k}\left(G_{N}\right)$ for integer $k \geq 0$ and real $\alpha$, where $V_{q, \alpha}^{k}\left(G_{i}\right)$ denotes the space of all distributions $u \in \mathcal{D}^{\prime}\left(G_{i}\right)$ satisfying $r^{\alpha / q+|\beta|-k}\left|D^{\beta} u_{i}\right| \in L_{q}\left(G_{i}\right), i=1, \ldots, N . \mathbf{V}_{q, \alpha}^{k}(G)$ is a Banach space for the norm

$$
\|u\|_{\mathbf{V}_{q, \alpha}^{k}(G)}=\sum_{i=1}^{N}\left(\int_{G_{i}|\beta|=0} \sum^{k} r^{\alpha+q(|\beta|-k)}\left|D^{\beta} u_{i}\right|^{q} d x\right)^{1 / q} .
$$

$\mathbf{V}_{q, \alpha}^{k-1 / q}(\partial G)$ is the space of functions $\varphi$ on $\partial G$ with the norm $\|\varphi\|_{\mathbf{V}_{q, \alpha}^{k-1 / q}(\partial G)}=$ $\inf \|\Phi\|_{\mathbf{V}_{q, \alpha}^{k}(G)}$, where the infimum is taken over all functions $\Phi$ such that $\left.\Phi\right|_{\partial G}=\varphi$ in the sense of traces. We write

$$
\mathbf{W}^{k}(G) \equiv \mathbf{W}^{k, 2}(G), \quad \stackrel{\circ}{\mathbf{W}}_{\alpha}^{k}(G) \equiv \mathbf{V}_{2, \alpha}^{k}(G), \quad \stackrel{\circ}{\mathbf{W}}_{\alpha}^{k-1 / 2}(\partial G) \equiv \mathbf{V}_{2, \alpha}^{k-1 / 2}(\partial G) .
$$

Definition 1. A function $u$ is called a weak solution of problem $(L)$ provided that $u \in \mathbf{C}^{0}(\bar{G}) \cap \stackrel{\circ}{\mathbf{W}}_{0}^{1}(G)$ and $u$ satisfies the integral identity

$$
\begin{aligned}
& \int_{G} a u_{x_{j}} \eta_{x_{j}} d x+\int_{\Sigma} \frac{1}{r} \beta(\phi) u(x) \eta(x) d s+\int_{\partial G} \alpha(x) \frac{1}{r} \gamma(\phi) u(x) \eta(x) d s \\
& =\int_{\partial G} \alpha(x) g(x) \eta(x) d s+\int_{\Sigma} h(x) \eta(x) d s-\int_{G}(p u(x)+f(x)) \eta(x) d x
\end{aligned}
$$

for all $\eta \in \mathbf{C}^{0}(\bar{G}) \cap \mathbf{W}_{0}^{1}(G)$ (summation over repeated indices from 1 to $n$ is understood); here

$$
\int_{G} f(x) d x=\sum_{i=1}^{N} \int_{G_{i}} f_{i}(x) d x, \quad \int_{\Sigma} h(x) d s=\sum_{k=1}^{N-1} \int_{\Sigma_{k}} h_{k}(x) d s, \quad \text { etc. }
$$

REMARK 1. We assume that $\left.u\right|_{\partial G \cap \mathcal{D}}=0$.

We assume that $M_{0}=\max _{x \in \bar{G}}|u(x)|$ is known.

Lemma 1.1. Let $u$ be a weak solution of $(L)$. For any $\eta \in \mathbf{C}^{0}(\bar{G}) \cap$ $\stackrel{\circ}{\mathbf{W}}_{0}^{1}(G)$, 


$$
\begin{aligned}
& (\mathrm{II})_{\mathrm{loc}} \quad \int_{G_{0}^{\varrho}}\left\{a u_{x_{i}} \eta_{x_{i}}+(f(x)+p u(x)) \eta(x)\right\} d x=\int_{\Omega_{\varrho}} a \frac{\partial u}{\partial r} \eta(x) d \Omega_{\varrho} \\
& +\int_{\Gamma_{0}^{\varrho}} \alpha(x)\left(g(x)-\frac{1}{r} \gamma(\phi) u(x)\right) \eta(x) d s+\int_{\Sigma_{0}^{\varrho}}\left(h(x)-\frac{1}{r} \beta(\phi) u(x)\right) \eta(x) d s
\end{aligned}
$$

for a.e. $\varrho \in(0, d)$.

Proof. The proof is analogous to the proof of [3, Lemma 5.2, pp. 167$170]$.

Define numbers

$$
\left\{\begin{array}{l}
a_{*}=\min \left\{a_{1}, \ldots, a_{N}\right\}>0, \quad a^{*}=\max \left\{a_{1}, \ldots, a_{N}\right\}>0 \\
p^{*}=\max \left\{p_{1}, \ldots, p_{N}\right\} \geq 0 \\
{[a]_{\Sigma_{k}}=a_{k}-a_{k+1}, k=1, \ldots, N-1} \\
a_{0}=\max _{1 \leq k \leq N-1}\left|[a]_{\Sigma_{k}}\right| ; \quad \widetilde{a}=\max \left(a^{*}, a_{0}\right) .
\end{array}\right.
$$

We assume that:

(a) $f \in \mathbf{L}_{q / 2}(G) \cap \mathbf{L}_{2}(G) ; q>n$;

(b) $\gamma(\phi) \geq \gamma_{0}>\widetilde{a} \tan \left(\omega_{0} / 2\right)$ on $\partial G ; \beta_{k}(\phi) \geq \beta_{0}>\widetilde{a} \tan \left(\omega_{0} / 2\right)$ on $\Sigma_{k}, k=1, \ldots, N-1$;

(c) there exist numbers $f_{0}, g_{0}, h_{0} \geq 0, s>1, \beta \geq s-2$ such that $|f(x)| \leq f_{0}|x|^{\beta}, \quad|g(x)| \leq g_{0}|x|^{s-1}, \quad\left|h_{k}(x)\right| \leq h_{0}|x|^{s-1}, \quad k=1, \ldots, N-1$.

Our main result is the following theorem. Let

$$
\lambda=\frac{2-n+\sqrt{(n-2)^{2}+4 \vartheta}}{2},
$$

where $\vartheta$ is the smallest positive eigenvalue of the problem (EVP) (see Subsection 2.2).

THEOREM 1.2. Let $u$ be a weak solution of problem $(L)$, and assume that conditions (a)-(c) are satisfied. Let $G$ and the parameters in (a)-(c) be such that $\lambda>1$. Then there are $d \in(0,1)$ and constants $C_{0}, c>0$ depending only on $n, a_{*}, a^{*}, p^{*}, \lambda, q, \omega_{0}, f_{0}, h_{0}, g_{0}, \beta_{0}, \gamma_{0}, s, M_{0}$, meas $G$, $\operatorname{diam} G$ such that for all $x \in G_{0}^{d}$,

$$
\begin{aligned}
|u(x)| \leq & C_{0}\left(\|u\|_{2, G}+f_{0}+\frac{1}{\sqrt{\gamma_{0}}} g_{0}+\frac{1}{\sqrt{\beta_{0}}} h_{0}\right) \\
& \times \begin{cases}|x|^{\lambda} & \text { if } s>\lambda, \\
|x|^{\lambda} \ln ^{c}(1 /|x|) & \text { if } s=\lambda, \\
|x|^{s} & \text { if } s<\lambda .\end{cases}
\end{aligned}
$$


Suppose, in addition, that

$$
\begin{gathered}
\gamma \in \mathbf{C}^{1}(\partial G), \quad f \in \mathbf{V}_{q, 2 q-n}^{0}(G), \quad h \in V_{q, 2 q-n}^{1-1 / q}(\Sigma), \\
g \in \mathbf{V}_{q, 2 q-n}^{1-1 / q}(\partial G), \quad q>n,
\end{gathered}
$$

and there is a number

$$
\tau_{s}:=\sup _{\varrho>0} \varrho^{-s}\left(\|h\|_{\mathbf{V}_{q, 2 q-n}^{1-1 / q}\left(\Sigma_{\varrho / 2}^{\varrho}\right)}+\|g\|_{V_{q, 2 q-n}^{1-1 / q}\left(\Gamma_{\varrho / 2}^{\varrho}\right)}\right) .
$$

Then for all $x \in G_{0}^{d}$,

$$
\begin{aligned}
|\nabla u(x)| \leq & C_{1}\left(\|u\|_{2, G}+f_{0}+\frac{1}{\sqrt{\gamma_{0}}} g_{0}+\frac{1}{\sqrt{\beta_{0}}} h_{0}+\tau_{s}\right) \\
& \times \begin{cases}|x|^{\lambda-1} & \text { if } s>\lambda, \\
|x|^{\lambda-1} \ln ^{c}(1 /|x|) & \text { if } s=\lambda, \\
|x|^{s-1} & \text { if } s<\lambda .\end{cases}
\end{aligned}
$$

Furthermore,

- $u \in \mathbf{V}_{q, 2 q-n}^{2}(G), q>n$ and

$$
\begin{aligned}
\|u\|_{\mathbf{V}_{q, 2 q-n}^{2}\left(G_{0}^{\varrho}\right) \leq} & C_{2}\left(\|u\|_{2, G}+f_{0}+\frac{1}{\sqrt{\gamma_{0}}} g_{0}+\frac{1}{\sqrt{\beta_{0}}} h_{0}+\tau_{s}\right) \\
& \times \begin{cases}\varrho^{\lambda} & \text { if } s>\lambda, \\
\varrho^{\lambda} \ln ^{c}(1 / \varrho) & \text { if } s=\lambda, \\
\varrho^{s} & \text { if } s<\lambda ;\end{cases}
\end{aligned}
$$

- if $f \in \stackrel{\circ}{\mathbf{W}}_{\alpha}^{0}(G), \int_{\Sigma} r^{\alpha-1} h^{2}(x) d s<\infty, \int_{\partial G} r^{\alpha-1} g^{2}(x) d s<\infty$, where

$$
4-n-2 \lambda<\alpha \leq 2 \text {, }
$$

then $u \in \stackrel{\circ}{\mathbf{W}}_{\alpha-2}^{1}(G)$ and

$$
\begin{aligned}
\int_{G} a\left(r^{\alpha-2}|\nabla u|^{2}+r^{\alpha-4}\right. & \left.u^{2}\right) d x+\int_{\Sigma} r^{\alpha-3} \beta(\phi) u^{2}(x) d s \\
& +\int_{\partial G} \alpha(x) r^{\alpha-3} \gamma(\phi) u^{2}(x) d s \\
\leq & C\left\{\int_{G}\left(u^{2}+\left(1+r^{\alpha}\right) f^{2}(x)\right) d x+\int_{\Sigma} r^{\alpha-1} h^{2}(x) d s\right. \\
& \left.+\int_{\partial G} \alpha(x) r^{\alpha-1} g^{2}(x) d s\right\},
\end{aligned}
$$

where the constant $C>0$ depends only on $q, n, a_{*}, a^{*}, \alpha, \lambda$ and the domain $G$. 


\section{Preliminaries}

2.1. Auxiliary formulae. Let us recall some well known formulae relating to spherical coordinates $\left(r, \omega_{1}, \ldots, \omega_{n-1}\right)$ centered at the conical point $\mathcal{O}$ :

- $d x=r^{n-1} d r d \Omega$,

- $d \Omega_{\varrho}=\varrho^{n-1} d \Omega$,

- $d \Omega=J(\phi) d \phi$ denotes the $(n-1)$-dimensional area element of the unit sphere,

- $J(\phi)=\sin ^{n-2} \phi_{1} \sin ^{n-3} \phi_{2} \ldots \sin \phi_{n-2}$,

- $d \phi=d \phi_{1} \ldots d \phi_{n-1}$,

- $d s$ denotes the $(n-1)$-dimensional area element on $\partial G$;

- $d \sigma$ denotes the $(n-2)$-dimensional area element on $\partial \Omega$;

- $d s=r^{n-2} d r d \sigma$;

- $|\nabla u|^{2}=\left(\frac{\partial u}{\partial r}\right)^{2}+\frac{1}{r^{2}}\left|\nabla_{\phi} u\right|^{2}$, where $\left|\nabla_{\phi} u\right|$ is the projection of the vector $\nabla u$ onto the tangent plane to the unit sphere at the point $\phi$,

- $\left|\nabla_{\phi} u\right|^{2}=\sum_{i=1}^{n-1} \frac{1}{q_{i}}\left(\frac{\partial u}{\partial \phi_{i}}\right)^{2}$, where $q_{1}=1$ and $q_{i}=\left(\sin \phi_{1} \cdots \sin \phi_{i-1}\right)^{2}$ for $i \geq 2$,

- $\Delta u=\frac{\partial^{2} u}{\partial r^{2}}+\frac{n-1}{r} \frac{\partial u}{\partial r}+\frac{1}{r^{2}} \Delta_{\phi} u$,

- $\Delta_{\phi} u=\frac{1}{J(\phi)} \sum_{i=1}^{n-1} \frac{\partial}{\partial \phi_{i}}\left(\frac{J(\phi)}{q_{i}} \frac{\partial u}{\partial \phi_{i}}\right)$, the Laplace-Beltrami operator,

- $\operatorname{div}_{\phi} \vec{u}=\frac{1}{J(\phi)} \sum_{i=1}^{n-1} \frac{\partial}{\partial \phi_{i}}\left(\frac{J(\phi)}{q_{i}} u_{i}\right)$.

$C=C(\ldots), c=c(\ldots)$ denote constants depending only on the quantities appearing in parentheses. Hereafter, the same letters $C, c$ will (generally) be used to denote different constants depending on the same set of arguments.

By a direct calculation we obtain

LEMMA 2.1.

$$
\begin{aligned}
& \left.x_{i} \cos \left(\vec{n}, x_{i}\right)\right|_{\Gamma_{0}^{d}}=0,\left.\quad \cos \left(\vec{n}, x_{1}\right)\right|_{\Gamma_{0}^{d}}=-\sin \frac{\omega_{0}}{2}, \\
& \left.x_{i} \cos \left(\vec{n}_{k}, x_{i}\right)\right|_{\Sigma_{k}}=0, \quad k=1, \ldots, N-1
\end{aligned}
$$

(the latter is the equation of $\Sigma_{k}$ ).

2.2. Auxiliary inequalities. We need some statements and inequalities.

The eigenvalue problem. Let $\Omega \subset S^{n-1}$ with smooth boundary $\partial \Omega$ be the intersection of the cone $\mathcal{C}$ with $S^{n-1}$. Let $\vec{\nu}$ be the exterior normal to $\partial \mathcal{C}$ at points of $\partial \Omega$ and $\vec{\tau}_{k}$ be the exterior normal to $\Sigma_{k}$ with respect to $\Omega_{k}$ (lying in the tangent plane to $\Omega_{k}$ ), $k=1, \ldots, N-1$. Let $\gamma(\phi), \phi \in \partial \Omega$, be a positive bounded piecewise smooth function, and $\beta_{k}(\phi)$ be a positive continuous function on $\Sigma_{k}, k=1, \ldots, N-1$. We consider the eigenvalue 
problem for the Laplace-Beltrami operator $\Delta_{\phi}$ on the unit sphere $(\mathrm{EVP})\left\{\begin{array}{l}a_{i}\left(\Delta_{\phi} \psi_{i}+\vartheta \psi_{i}\right)=0, \quad \phi \in \Omega_{i}, a_{i} \text { are positive constants; } i=1, \ldots, N, \\ {[\psi]_{\sigma_{k}}=0, \quad\left[a \frac{\partial \psi}{\partial \vec{\tau}_{k}}\right]_{\sigma_{k}}+\left.\beta_{k}(\phi) \psi\right|_{\sigma_{k}}=0, \quad k=1, \ldots, N-1,} \\ \alpha(\phi) a \frac{\partial \psi}{\partial \vec{\nu}}+\left.\gamma(\phi) \psi\right|_{\partial \Omega}=0,\end{array}\right.$

which is to determine all values $\vartheta$ (eigenvalues) for which (EVP) has nonzero weak solutions (eigenfunctions).

From the variational principle we obtain the Friedrichs-Wirtinger inequality (the proof is analogous to one in $\S 2.4$ of [3]):

THEOREM 2.2. Let $\vartheta$ be the smallest positive eigenvalue of problem (EVP). Let $\Omega \subset S^{n-1}$. Let $\psi \in \mathbf{W}^{1}(\Omega)$ satisfy the boundary and conjunction conditions from (EVP) in the weak sense. Let $\gamma(\phi)$ be a positive bounded piecewise smooth function on $\partial \Omega$, and $\beta_{k}(\phi)$ be a positive continuous function on $\sigma_{k}$, $k=1, \ldots, N-1$. Then

$$
\begin{aligned}
\vartheta \int_{\Omega} a \psi^{2}(\phi) d \Omega \leq & \int_{\Omega} a\left|\nabla_{\phi} \psi\right|^{2} d \Omega+\sum_{k=1}^{N-1} \int_{\sigma_{k}} \beta_{k}(\phi) \psi^{2}(\phi) d \sigma \\
& +\int_{\partial \Omega} \alpha(\phi) \gamma(\phi) \psi^{2}(\phi) d \sigma .
\end{aligned}
$$

Because of (1.3), the Friedrichs-Wirtinger inequality will be written in the following form:

$$
\begin{aligned}
\lambda(\lambda+n-2) \int_{\Omega} a \psi^{2}(\phi) d \Omega \leq & \int_{\Omega} a\left|\nabla_{\phi} \psi\right|^{2} d \Omega+\int_{\sigma} \beta(\phi) \psi^{2}(\phi) d \sigma \\
& +\int_{\partial \Omega} \alpha(\phi) \gamma(\phi) \psi^{2}(\phi) d \sigma
\end{aligned}
$$

for all $\psi(\phi) \in \mathbf{W}^{1}(\Omega)$ satisfying the boundary and conjunction conditions from (EVP) in the weak sense. Multiplying (2.3) by $r^{n-5+\alpha}$ and integrating over $r \in(0, d)$ we obtain

COROLlary 2.3 .

$$
\begin{array}{r}
\int_{G_{0}^{d}} a r^{\alpha-4} u^{2} d x \leq \frac{1}{\lambda(\lambda+n-2)}\left\{\int_{G_{0}^{d}} a r^{\alpha-2}|\nabla u|^{2} d x+\int_{\Sigma_{0}^{d}} r^{\alpha-3} \beta(\phi) u^{2}(x) d s\right. \\
\left.+\int_{\Gamma_{0}^{d}} \alpha(x) r^{\alpha-3} \gamma(\phi) u^{2}(x) d s\right\}, \quad \forall \alpha,
\end{array}
$$

for all $u \in \stackrel{\circ}{\mathbf{W}}_{\alpha-2}^{1}\left(G_{0}^{d}\right)$ such that $u(\cdot, \omega)$ satisfies the boundary and conjunction conditions from (EVP) in the weak sense. 
Now we use the Hardy inequality (see [8, Theorem 330]) to get:

Proposition 2.4 (Hardy-Friedrichs-Wirtinger inequality). Let $u \in$ $\mathbf{C}^{0}(\bar{G}) \cap \stackrel{\circ}{\mathbf{W}}_{\alpha-2}^{1}(G)$ with $u(0)=0$ and suppose $u(\cdot, \omega)$ satisfies the boundary and conjunction conditions from (EVP) in the weak sense. Let $\beta_{k}(\phi), \gamma(\phi)$ be positive bounded piecewise smooth functions. Then

$$
\begin{aligned}
\int_{G_{0}^{d}} a r^{\alpha-4} u^{2} d x \leq & H(\lambda, n, \alpha)\left\{\int_{G_{0}^{d}} a r^{\alpha-2}|\nabla u|^{2} d x\right. \\
& \left.+\int_{\Sigma_{0}^{d}} r^{\alpha-3} \beta(\phi) u^{2}(x) d s+\int_{\Gamma_{0}^{d}} r^{\alpha-3} \alpha(x) \gamma(\phi) u^{2}(x) d s\right\}, \\
H(\lambda, n, \alpha)= & \frac{1}{\lambda(\lambda+n-2)+\frac{1}{4}(4-n-\alpha)^{2}}, \quad \alpha \leq 4-n .
\end{aligned}
$$

The proof is similar to that in $[3, \S 2.5 .2]$.

Lemma 2.5. Let $G_{0}^{d}$ be a conical domain and $\nabla u(\varrho, \cdot) \in \mathbf{L}_{2}(\Omega)$ a.e. $\varrho \in$ $(0, d)$. Assume that

$$
\begin{aligned}
U(\varrho)= & \int_{G_{o}^{\varrho}} a r^{2-n}|\nabla u|^{2} d x+\int_{\Sigma_{0}^{\varrho}} r^{1-n} \beta(\phi) u^{2}(x) d s \\
& +\int_{\Gamma_{0}^{\varrho}} r^{1-n} \gamma(\phi) u^{2}(x) d s<\infty, \quad \varrho \in(0, d) .
\end{aligned}
$$

Then

$$
\left.\int_{\Omega} a\left(\varrho u \frac{\partial u}{\partial r}+\frac{n-2}{2} u^{2}\right)\right|_{r=\varrho} d \Omega \leq \frac{\varrho}{2 \lambda} U^{\prime}(\varrho) .
$$

Proof. Writing $U(\varrho)$ in spherical coordinates,

$$
\begin{aligned}
U(\varrho)= & \int_{0}^{\varrho} r^{2-n}\left(\int_{\Omega} a|\nabla u|^{2} d \Omega\right) r^{n-1} d r \\
& +\int_{0}^{\varrho} r^{1-n}\left(\int_{\Sigma} \beta(\phi)|u|^{2} d \sigma+\int_{\partial \Omega} \gamma(\phi)|u|^{2} d \sigma\right) r^{n-2} d r \\
= & \int_{0}^{\varrho} r \int_{\Omega} a\left(u_{r}^{2}+\frac{1}{r^{2}}\left|\nabla_{\phi} u\right|^{2}\right) d \Omega d r \\
& +\int_{0}^{\varrho} \frac{1}{r}\left(\int_{\Sigma} \beta(\phi)|u|^{2} d \sigma+\int_{\partial \Omega} \gamma(\phi)|u|^{2} d \sigma\right) d r
\end{aligned}
$$


and differentiating with respect to $\varrho$ we obtain

$$
\begin{aligned}
U^{\prime}(\varrho)= & \left.\int_{\Omega} a\left(\varrho\left(\frac{\partial u}{\partial r}\right)^{2}+\frac{1}{\varrho}\left|\nabla_{\phi} u\right|^{2}\right)\right|_{r=\varrho} d \Omega \\
& +\frac{1}{\varrho}\left(\int_{\Sigma} \beta(\phi) u^{2}(\varrho, \phi) d \sigma+\int_{\partial \Omega} \gamma(\phi) u^{2}(\varrho, \phi) d \sigma\right) .
\end{aligned}
$$

Moreover, by Cauchy's inequality, we have $\varrho u \frac{\partial u}{\partial r} \leq \frac{\varepsilon}{2} u^{2}+\frac{1}{2 \varepsilon} \varrho^{2}\left(\frac{\partial u}{\partial r}\right)^{2}$ for all $\varepsilon>0$. Then

$$
\left.\int_{\Omega} a\left(\varrho u \frac{\partial u}{\partial r}+\frac{n-2}{2} u^{2}\right)\right|_{r=\varrho} d \Omega \leq \frac{\varepsilon+n-2}{2} \int_{\Omega} a u^{2} d \Omega+\frac{\varrho^{2}}{2 \varepsilon} \int_{\Omega} a\left(\frac{\partial u}{\partial r}\right)^{2} d \Omega .
$$

Thus choosing $\varepsilon=\lambda$ we obtain, by the Friedrichs-Wirtinger inequality (2.3),

$$
\begin{aligned}
\int_{\Omega} a\left(\varrho u \frac{\partial u}{\partial r}\right. & \left.+\frac{n-2}{2} u^{2}\right)\left.\right|_{r=\varrho} d \Omega \\
\leq & \frac{\varepsilon+n-2}{2 \lambda(\lambda+n-2)} \int_{\Omega} a\left|\nabla_{\phi} u\right|^{2} d \Omega+\frac{\varrho^{2}}{2 \varepsilon} \int_{\Omega} a\left(\frac{\partial u}{\partial r}\right)^{2} d \Omega \\
& +\frac{\varepsilon+n-2}{2 \lambda(\lambda+n-2)}\left(\int_{\Sigma} \beta(\phi) u^{2}(\varrho, \phi) d \sigma+\int_{\partial \Omega} \gamma(\phi) u^{2}(\varrho, \phi) d \sigma\right) \\
= & \frac{\varrho}{2 \lambda} U^{\prime}(\varrho) .
\end{aligned}
$$

We also need the well known inequalities

$$
\begin{array}{ll}
\int_{\Gamma} v d s \leq C \int_{G}(|v|+|\nabla v|) d x, & \forall v \in \mathbf{W}^{1,1}(G), \forall \Gamma \subseteq \partial G, \\
\int_{\partial G} v^{2} d s \leq \int_{G}\left(\delta|\nabla v|^{2}+\frac{1}{\delta} c_{0} v^{2}\right) d x, & \forall v \in \mathbf{W}^{1,2}(G), \forall \delta>0 .
\end{array}
$$

2.3. Quasi-distance $r_{\varepsilon}(x)$. Further, we define a function $r_{\varepsilon}(x)$ as follows. We fix $Q=(-1,0, \ldots, 0) \in S^{n-1} \backslash \bar{\Omega}$ and consider the unit radius-vector $\vec{l}=\mathcal{O} Q=\{-1,0, \ldots, 0\}$. We denote by $\vec{r}$ the radius-vector of the point $x \in \bar{G}$ and introduce the vector $\vec{r}_{\varepsilon}=\vec{r}-\varepsilon \vec{l}$ for $\varepsilon>0$. Since $\varepsilon \vec{l} \notin G_{0}^{d}$ for all $\varepsilon \in(0, d)$, it follows that $r_{\varepsilon}(x)=|\vec{r}-\varepsilon \vec{l}| \neq 0$ for all $x \in \bar{G}$. It is easy to see that $r_{\varepsilon}(x)$ has the following properties:

1. There exists $h>0$ such that $r_{\varepsilon}(x) \geq h r$ and $r_{\varepsilon}(x) \geq h \varepsilon$ for all $x \in \bar{G}$, where

$$
h= \begin{cases}1 & \text { if } x_{1} \geq 0, \\ \sin \left(\omega_{0} / 2\right) & \text { if } x_{1}<0 .\end{cases}
$$


Proof. From the definition of $r_{\varepsilon}(x)$ we know that

$$
r_{\varepsilon}^{2}=\left(x_{1}+\varepsilon\right)^{2}+\sum_{i=2}^{n} x_{i}^{2}=\left(x_{1}+\varepsilon\right)^{2}+r^{2}-x_{1}^{2}=r^{2}+2 \varepsilon x_{1}+\varepsilon^{2} .
$$

If $x_{1} \geq 0$ we obtain either $r_{\varepsilon}^{2} \geq r^{2}$, so $r_{\varepsilon} \geq r$, or $r_{\varepsilon}^{2} \geq \varepsilon^{2}$, so $r_{\varepsilon} \geq \varepsilon$.

If $x_{1}=r \cos \phi \leq 0, \phi \in\left[\pi / 2, \omega_{0} / 2\right]$, we obtain by the Cauchy inequality: either

$|2 \varepsilon r \cos \phi| \leq r^{2} \cos ^{2} \phi+\varepsilon^{2} \Rightarrow 2 \varepsilon r \cos \phi \geq-r^{2} \cos ^{2} \phi-\varepsilon^{2} \Rightarrow r_{\varepsilon} \geq r \sin \left(\omega_{0} / 2\right)$

or

$|2 \varepsilon r \cos \phi| \leq \varepsilon^{2} \cos ^{2} \phi+r^{2} \Rightarrow 2 \varepsilon r \cos \phi \geq-r^{2}-\varepsilon^{2} \cos ^{2} \phi \Rightarrow r_{\varepsilon} \geq \varepsilon \sin \left(\omega_{0} / 2\right)$.

2. If $x \in G_{d}$, then $r_{\varepsilon}(x) \geq d / 2$ for all $\varepsilon \in(0, d / 2)$,

3. $\lim _{\varepsilon \rightarrow 0^{+}} r_{\varepsilon}(x)=r$ for all $x \in \bar{G}$.

4. $\left|\nabla r_{\varepsilon}\right|^{2}=1$ and $\Delta r_{\varepsilon}=(n-1) / r_{\varepsilon}$.

Lemma 2.6. Let $v \in \mathbf{C}^{0}(\bar{G}) \cap \mathbf{W}^{1}(G)$ with $v(0)=0$ and suppose $v(\cdot, \omega)$ satisfies the boundary and conjunction conditions from (EVP) in the weak sense. Let $\beta(\phi), \gamma(\phi)>0$. Then for any $\varepsilon>0$,

$$
\begin{aligned}
\int_{G_{0}^{d}} a r_{\varepsilon}^{\alpha-4} v^{2} d x & \leq H(\lambda, n, \alpha)\left\{\int_{G_{0}^{d}} a r_{\varepsilon}^{\alpha-2}|\nabla v|^{2} d x\right. \\
& \left.+\int_{\Sigma_{0}^{d}} r_{\varepsilon}^{\alpha-3} \beta(\phi) v^{2}(x) d s+\int_{\Gamma_{0}^{d}} r_{\varepsilon}^{\alpha-3} \alpha(x) \gamma(\phi) v^{2}(x) d s\right\},
\end{aligned}
$$

where $H(\lambda, n, \alpha)$ is defined by $(2.5)$.

LEMMA 2.7. Let $v \in \mathbf{C}^{0}(\bar{G}) \cap \mathbf{W}^{1}(G)$ and suppose that $v(\cdot, \omega)$ satisfies the boundary and conjunction conditions from (EVP) in the weak sense. Let $\beta(\phi), \gamma(\phi)>0$. Then for any $\varepsilon>0$,

$$
\begin{aligned}
& \int_{G_{0}^{d}} a r_{\varepsilon}^{\alpha-2} r^{-2} v^{2} d x \leq \frac{1}{\lambda(\lambda+n-2)}\left\{\int_{G_{0}^{d}} a r_{\varepsilon}^{\alpha-2}|\nabla v|^{2} d x\right. \\
& \left.\quad+\int_{\Sigma_{0}^{d}} r^{-1} r_{\varepsilon}^{\alpha-2} \beta(\phi) v^{2}(x) d s+\int_{\Gamma_{0}^{d}} r^{-1} r_{\varepsilon}^{\alpha-2} \alpha(x) \gamma(\phi) v^{2} d s\right\} .
\end{aligned}
$$

For the proof of these lemmas see Lemmas 2.37 and 2.32 in [3].

2.4. The Cauchy problem for a differential inequality

THEOREM 2.8. Let $U$ be an increasing, nonnegative differentiable function defined on $[0,2 d]$ and satisfying

$$
\left\{\begin{array}{l}
U^{\prime}(\varrho)-\mathcal{P}(\varrho) U(\varrho)+\mathcal{N}(\varrho) U(2 \varrho)+\mathcal{Q}(\varrho) \geq 0, \quad 0<\varrho<d \\
U(d) \leq U_{0}
\end{array}\right.
$$


where $\mathcal{P}(\varrho), \mathcal{N}(\varrho), \mathcal{Q}(\varrho)$ are nonnegative continuous functions defined on $[0,2 d]$ and $U_{0}$ is a constant. Then

$$
\begin{aligned}
U(\varrho) \leq & \exp \left(\int_{\varrho}^{d} \mathcal{B}(\tau) d \tau\right)\left\{U_{0} \exp \left(-\int_{\varrho}^{d} \mathcal{P}(\tau) d \tau\right)\right. \\
& \left.+\int_{\varrho}^{d} \mathcal{Q}(\tau) \exp \left(-\int_{\varrho}^{\tau} \mathcal{P}(\sigma) d \sigma\right) d \tau\right\}
\end{aligned}
$$

with

$$
\mathcal{B}(\varrho)=\mathcal{N}(\varrho) \exp \left(\int_{\varrho}^{2 \varrho} \mathcal{P}(\sigma) d \sigma\right) .
$$

Proof. See [3, §1.10, Theorem 1.57].

2.5. The comparison principle. We consider the second order linear degenerate operator $Q$ of the form

$$
\begin{gathered}
Q(u, \eta) \equiv \int_{G_{0}^{d}}\left\{a u_{x_{i}} \eta_{x_{i}}+(f(x)+p u(x)) \eta(x)\right\} d x-\int_{\Omega_{d}} a \frac{\partial u}{\partial r} \eta(x) d \Omega_{d} \\
-\int_{\Gamma_{0}^{d}} \alpha(x)\left(g(x)-\frac{1}{r} \gamma(\phi) u(x)\right) \eta(x) d s-\int_{\Sigma_{0}^{d}}\left(h(x)-\frac{1}{r} \beta(\phi) u(x)\right) \eta(x) d s
\end{gathered}
$$

for $u \in \mathbf{C}^{0}\left(\overline{G_{0}^{d}}\right) \cap \stackrel{\circ}{\mathbf{W}}_{0}^{1}\left(G_{0}^{d}\right)$ and nonnegative $\eta \in \mathbf{C}^{0}\left(\overline{G_{0}^{d}}\right) \cap \stackrel{\circ}{\mathbf{W}}_{0}^{1}\left(G_{0}^{d}\right)$.

Proposition 2.9. Let $\beta(\phi), \gamma(\phi)$ be positive piecewise smooth functions, $0<a_{*} \leq a \leq a^{*}, p \geq 0$ and $d \ll 1$. Let $u, w \in \mathbf{C}^{0}\left(\overline{G_{0}^{d}}\right) \cap \stackrel{\circ}{\mathbf{W}}_{0}^{1}\left(G_{0}^{d}\right)$ satisfy

$$
Q(u, \eta) \leq Q(w, \eta)
$$

for all nonnegative $\eta \in \mathbf{C}^{0}\left(\overline{G_{0}^{d}}\right) \cap \stackrel{\circ}{\mathbf{W}}_{0}^{1}\left(G_{0}^{d}\right)$ and also

$$
u(x) \leq w(x), \quad x \in \Omega_{d} \cup\left(\Gamma_{0}^{d} \cap \mathcal{D}\right),
$$

in the weak sense. Then $u(x) \leq w(x)$ in $G_{0}^{d}$.

Proof. Define $z(x)=u(x)-w(x)$. Then

$$
\begin{aligned}
0 \geq & Q(u, \eta)-Q(w, \eta)=\int_{G_{0}^{d}}\left\{a z_{x_{i}} \eta_{x_{i}}+p z(x) \eta(x)\right\} d x \\
& +\int_{\Gamma_{0}^{d}} \alpha(x) \frac{1}{r} \gamma(\phi) z(x) \eta(x) d s+\int_{\Sigma_{0}^{d}} \frac{1}{r} \beta(\phi) z(x) \eta(x) d s \\
& -\int_{\Omega_{d}} a \frac{\partial z}{\partial r} \eta(x) d \Omega_{d}
\end{aligned}
$$


for all nonnegative $\eta \in \mathbf{C}^{0}\left(\overline{G_{0}^{d}}\right) \cap \stackrel{\circ}{\mathbf{W}}_{0}^{1}\left(G_{0}^{d}\right)$. Define

$$
\begin{aligned}
& \left(G_{0}^{d}\right)^{+}:=\left\{x \in G_{0}^{d} \mid u(x)>w(x)\right\} \subset G_{0}^{d}, \\
& \left(\Sigma_{0}^{d}\right)^{+}:=\left\{x \in \Sigma_{0}^{d} \mid u(x)>w(x)\right\} \subset \Sigma_{0}^{d}, \\
& \left(\Gamma_{0}^{d}\right)^{+}:=\left\{x \in \Gamma_{0}^{d} \mid u(x)>w(x)\right\} \subset \Gamma_{0}^{d},
\end{aligned}
$$

and assume that $\left(G_{0}^{d}\right)^{+} \neq \emptyset$. As a test function in the integral inequality (2.18), we choose $\eta=\max \{(u-w), 0\}$. Then it follows from (2.17) and (2.18) that

$$
\int_{\left(G_{0}^{d}\right)^{+}}\left(a|\nabla z|^{2}+p z^{2}(x)\right) d x+\int_{\left(\Gamma_{0}^{d}\right)^{+}} \alpha(x) \frac{1}{r} \gamma(\phi) z^{2}(x) d s+\int_{\left(\Sigma_{0}^{d}\right)^{+}} \frac{1}{r} \beta(\phi) z^{2}(x) d s \leq 0 .
$$

This implies that $z(x)=0$ almost everywhere in $\left(G_{0}^{d}\right)^{+}$, contrary to our assumption that $\left(G_{0}^{d}\right)^{+} \neq \emptyset$. Thus Proposition 2.9 is proved.

3. The barrier function. A preliminary estimate of the modulus of the solution. Consider the linear operators $\mathcal{L}_{i} \equiv a_{i} \Delta, i=1, \ldots, N$, with the numbers (1.2).

Lemma 3.1 (Existence of the barrier function). Fix numbers $\beta_{0}, \gamma_{0}>$ $\widetilde{a} \tan \left(\omega_{0} / 2\right), \delta>0, g_{0}, h_{0} \geq 0$ and let $\gamma(\phi) \geq \gamma_{0}$ on $\partial G, \beta_{k}(\phi) \geq \beta_{0}$ on $\Sigma_{k}, k=1, \ldots, N-1$. There exists $m>0$, depending only on $\omega_{0}, a$ number $\varkappa_{0} \in\left(0,\left(\delta_{0} / \widetilde{a}\right) \cot \left(\omega_{0} / 2\right)-1\right)$ (where $\left.\delta_{0}=\min \left(\beta_{0}, \gamma_{0}\right)\right)$, numbers $B>0$ and $d \in(0,1)$ and a function $w \in \mathbf{C}^{1}\left(\bar{G}_{0}\right) \cap \mathbf{C}^{2}\left(G_{0}\right)$ that depend only on $\omega_{0}$, the ellipticity constants $a_{*}, a^{*}$ of the operators $\mathcal{L}_{i}$ and the quantities $\gamma_{0}, \beta_{0}, \delta_{0}, g_{0}, h_{0}, \omega_{0}$ such that for any $\varkappa \in\left(0, \min \left(\delta, \varkappa_{0}\right)\right)$ the following hold:

$$
\begin{gathered}
\mathcal{L}[w(x)] \leq-a_{*} m^{2}|x|^{\varkappa-1}, \quad x \in G_{0}^{d} \cap G_{ \pm} ; \\
\mathcal{B}[w(x)] \geq g_{0}|x|^{\delta}, \quad x \in \Gamma_{0}^{d} ; \\
\mathcal{S}_{k}[w(x)] \geq h_{0}|x|^{\delta}, \quad x \in \Sigma_{k}^{d}, k=1, \ldots, N-1 ; \\
0 \leq w(x) \leq c_{0}\left(\varkappa_{0}, B, \omega_{0}\right)|x|^{\varkappa+1}, \quad x \in \overline{G_{0}^{d}} ; \\
|\nabla w(x)| \leq c_{1}\left(\varkappa_{0}, B, \omega_{0}\right)|x|^{\varkappa}, \quad x \in \overline{G_{0}^{d}} .
\end{gathered}
$$

Proof. Let $\left(x, y, x^{\prime}\right) \in \mathbb{R}^{n}$, where $x=x_{1}, y=x_{2}, x^{\prime}=\left(x_{3}, \ldots, x_{n}\right)$. In $\left\{x_{1} \geq 0\right\}$ we consider the cone $K$ with vertex $\mathcal{O}$ such that $K \supset G_{0}^{d}$ (we recall that $\left.G_{0}^{d} \subset\left\{x_{1} \geq 0\right\}\right)$. Let $\partial K$ be the lateral surface of $K$ and let $\partial K \cap y \mathcal{O} x=\Gamma_{ \pm}$be $x= \pm m y$, where $m=\cot \left(\omega_{0} / 2\right), 0<\omega_{0}<\pi$, such that $x>m|y|$ in the interior of $K$. We shall consider the function

$$
w\left(x ; y, x^{\prime}\right) \equiv x^{\varkappa-1}\left(x^{2}-m^{2} y^{2}\right)+B x^{\varkappa+1}
$$


Let us calculate the action of $\mathcal{L}_{i}$ on the function (3.6). For $t=y / x,|t|<1 / h$ we obtain $\mathcal{L}_{i} w=-m^{2} x^{\varkappa-1} \varsigma_{i}(\varkappa)$, where $\varsigma_{i}(\varkappa)=a_{i}\left\{\left(\varkappa^{2}-3 \varkappa+2\right) t^{2}+2-\right.$ $\left.m^{-2}(1+B)\left(\varkappa^{2}+\varkappa\right)\right\}$. Since $\varsigma_{i}(0)=2 a_{i}\left(t^{2}+1\right) \geq 2 a_{i} \geq 2 a_{*}$ and since $\varsigma_{i}(\varkappa)$ are quadratic polynomials there exists a number $\varkappa_{0}>0$ depending only on $a_{*}, a^{*}, m$ such that $\varsigma_{i}(\varkappa) \geq a_{*}$ for $\varkappa \in\left[0, \varkappa_{0}\right]$. Therefore we obtain (3.1). Now, notice that

$$
\Gamma_{ \pm}: \quad x= \pm m y, \quad m=\cot \left(\omega_{0} / 2\right), 0<\omega_{0}<\pi .
$$

Then

$$
\begin{gathered}
\text { on } \Gamma_{+}: \quad\left\{\begin{array} { l } 
{ x = r \operatorname { c o s } ( \omega _ { 0 } / 2 ) , } \\
{ y = r \operatorname { s i n } ( \omega _ { 0 } / 2 ) , }
\end{array} \quad \left\{\begin{array}{l}
\angle(\vec{n}, x)=\pi / 2+\omega_{0} / 2, \\
\angle(\vec{n}, y)=\omega_{0} / 2,
\end{array}\right.\right. \\
\text { on } \Gamma_{-}: \quad\left\{\begin{array} { l } 
{ x = r \operatorname { c o s } ( \omega _ { 0 } / 2 ) , } \\
{ y = - r \operatorname { s i n } ( \omega _ { 0 } / 2 ) , }
\end{array} \quad \left\{\begin{array}{l}
\angle(\vec{n}, x)=\pi / 2+\omega_{0} / 2, \\
\angle(\vec{n}, y)=\pi+\omega_{0} / 2,
\end{array}\right.\right. \\
\sin \frac{\omega_{0}}{2}=\frac{1}{\sqrt{1+m^{2}}}, \quad \cos \frac{\omega_{0}}{2}=\frac{m}{\sqrt{1+m^{2}}} .
\end{gathered}
$$

Therefore

$$
\begin{aligned}
w_{x}= & (1+\varkappa) x^{\varkappa}(1+B)-(\varkappa-1) m^{2} y^{2} x^{\varkappa-2} \\
& \left.\Rightarrow w_{x}\right|_{\Gamma_{ \pm}}=[2+B(1+\varkappa)] x^{\varkappa} \\
w_{y}= & -\left.2 m^{2} y x^{\varkappa-1} \Rightarrow w_{y}\right|_{\Gamma_{ \pm}}=\mp 2 m x^{\varkappa} .
\end{aligned}
$$

Because of $\left.\frac{\partial w}{\partial \vec{n}}\right|_{\Gamma_{ \pm}}=\left.w_{x} \cos \angle(\vec{n}, x)\right|_{\Gamma_{ \pm}}+\left.w_{y} \cos \angle(\vec{n}, y)\right|_{\Gamma_{ \pm}}$and (3.8)-(3.11), we get

$$
\left.\frac{\partial w}{\partial \vec{n}}\right|_{\Gamma_{ \pm}}=-r^{\varkappa} \frac{m^{\varkappa}}{\left(1+m^{2}\right)^{(\varkappa+1) / 2}}\left\{2\left(1+m^{2}\right)+B(1+\varkappa)\right\} .
$$

Hence, by (3.7) and $\gamma(\phi) \geq \gamma_{0}>\widetilde{a} \tan \left(\omega_{0} / 2\right)$ on $\partial G$, it follows that

$$
\begin{aligned}
\left.\mathcal{B}[w]\right|_{\Gamma_{ \pm}^{d}} & =\frac{m^{\varkappa}}{\left(1+m^{2}\right)^{(\varkappa+1) / 2}} r^{\varkappa}\left\{B m \gamma(\phi)-\alpha(x) a\left(B(1+\varkappa)+2\left(1+m^{2}\right)\right)\right\} \\
& \geq \frac{m^{\varkappa}}{\left(1+m^{2}\right)^{(\varkappa+1) / 2}} r^{\varkappa}\left\{B m \gamma_{0}-B a^{*}(1+\varkappa)-2 a^{*}\left(1+m^{2}\right)\right\} .
\end{aligned}
$$

Since $m>a^{*} / \gamma_{0}$ and $\varkappa_{0}<m \gamma_{0} / a^{*}-1$, for $\varkappa \leq \varkappa_{0}$ we obtain

$$
\begin{aligned}
\left.\mathcal{B}[w]\right|_{\Gamma_{ \pm}^{d}} & \geq \frac{m^{\varkappa 0} r^{\varkappa}}{\left(1+m^{2}\right)^{\left(\varkappa_{0}+1\right) / 2}}\left\{B\left(m \gamma_{0}-a^{*}-a^{*} \varkappa_{0}\right)-2 a^{*}\left(1+m^{2}\right)\right\} \\
& \geq g_{0} r^{\delta}, \quad r \in(0, d),
\end{aligned}
$$

if we choose $\varkappa \leq \delta$, so $r^{\varkappa} \geq r^{\delta}$, and

$$
B \geq\left\{\frac{g_{0}\left(1+m^{2}\right)^{\left(\varkappa_{0}+1\right) / 2}}{m^{\varkappa_{0}}}+2 a^{*}\left(1+m^{2}\right)\right\} \cdot \frac{1}{m \gamma_{0}-a^{*}\left(1+\varkappa_{0}\right)} .
$$

Thus, (3.2) is proved. 
Now we prove (3.3). First, on $\Sigma_{k}$ we have $x=r \cos \left(\omega_{0} / 2-\theta_{k}\right), y=$ $r \sin \left(\omega_{0} / 2-\theta_{k}\right)$, so $y=m_{k} x$, where $m_{k}=\tan \left(\omega_{0} / 2-\theta_{k}\right)$. Therefore

$$
\begin{aligned}
\left.w\right|_{\Sigma_{k}} & =\left(B+1-m^{2} m_{k}^{2}\right) x^{1+\varkappa}=\left(B+1-m^{2} m_{k}^{2}\right) r^{1+\varkappa} \cos ^{1+\varkappa}\left(\omega_{0} / 2-\theta_{k}\right) \\
& =\frac{B+1-m^{2} m_{k}^{2}}{\left(1+m_{k}^{2}\right)^{(1+\varkappa) / 2}} r^{1+\varkappa} .
\end{aligned}
$$

Further,

$$
\begin{aligned}
& \cos \left(\vec{n}_{k}, x\right)=\cos \left(\pi / 2-\omega_{0} / 2+\theta_{k}\right)=\sin \left(\omega_{0} / 2-\theta_{k}\right)=\frac{m_{k}}{\sqrt{1+m_{k}^{2}}} \\
& \cos \left(\vec{n}_{k}, y\right)=\cos \left(\pi-\omega_{0} / 2+\theta_{k}\right)=-\cos \left(\omega_{0} / 2-\theta_{k}\right)=-\frac{1}{\sqrt{1+m_{k}^{2}}}
\end{aligned}
$$

on $\Sigma_{k}, k=1, \ldots, N-1$, and in virtue of

$$
\left.w_{x}\right|_{\Sigma_{k}}=\frac{(1+\varkappa)(1+B)+(1-\varkappa) m^{2} m_{k}^{2}}{\left(1+m_{k}^{2}\right)^{\varkappa / 2}} \cdot r^{\varkappa},\left.\quad w_{y}\right|_{\Sigma_{k}}=-\frac{2 m^{2} m_{k}^{2}}{\left(1+m_{k}^{2}\right)^{\varkappa / 2}} \cdot r^{\varkappa},
$$

we obtain

$$
\left.\frac{\partial w}{\partial n_{k}}\right|_{\Sigma_{k}}=m_{k} \cdot \frac{(1+\varkappa)(1+B)+(1-\varkappa) m^{2} m_{k}^{2}+2 m^{2}}{\left(1+m_{k}^{2}\right)^{(1+\varkappa) / 2}} \cdot r^{\varkappa} .
$$

Thus,

$\mathcal{S}_{k}[w]=$

$$
\frac{\beta_{k}(\phi)\left(B+1-m^{2} m_{k}^{2}\right)+m_{k}[a]_{\Sigma_{k}}\left\{(1+\varkappa)(1+B)+(1-\varkappa) m^{2} m_{k}^{2}+2 m^{2}\right\}}{\left(1+m_{k}^{2}\right)^{(1+\varkappa) / 2}} \cdot r^{\varkappa} .
$$

Since $0<\omega_{1} \leq \theta_{k}<\omega_{0}$ for all $k=1, \ldots, N-1$, we have $\left|m_{k}\right|<\tan \left(\omega_{0} / 2\right)=$ $1 / m$ for all $k=1, \ldots, N-1$. Now, as $\beta_{k}(\phi) \geq \beta_{0}>\widetilde{a} \tan \left(\omega_{0} / 2\right)$ on $\Sigma_{k}$, $k=1, \ldots, N-1$, and $\varkappa_{0}<m \beta_{0} / a_{0}-1$, for $\varkappa \leq \varkappa_{0}$ we get

$$
\begin{aligned}
\mathcal{S}_{k}[w] & \geq\left\{\beta_{0} B m-a_{0}\left(\left(1+\varkappa_{0}\right) B+2\left(1+m^{2}\right)\right)\right\} \frac{m^{\varkappa_{0}}}{\left(1+m^{2}\right)^{\left(1+\varkappa_{0}\right) / 2}} \cdot r^{\varkappa} \\
& \geq r^{\delta} \cdot h_{0},
\end{aligned}
$$

if we choose $\varkappa \leq \delta$, so $r^{\varkappa} \geq r^{\delta}$, and

$$
B \geq\left\{\frac{h_{0}\left(1+m^{2}\right)^{\left(\varkappa_{0}+1\right) / 2}}{m^{\varkappa_{0}}}+2 a_{0}\left(1+m^{2}\right)\right\} \cdot \frac{1}{m \beta_{0}-a_{0}\left(1+\varkappa_{0}\right)} .
$$

Thus, (3.3) is proved.

Now we show (3.4). Let us rewrite the function (3.6) in spherical coordinates. Recalling that $h=\cot \left(\omega_{0} / 2\right)$, we obtain 


$$
\begin{aligned}
w\left(x ; y, x^{\prime}\right) & =(1+B)(r \cos \phi)^{1+\varkappa}-h^{2} r^{2} \sin ^{2} \phi(r \cos \phi)^{\varkappa-1} \\
& =r^{1+\varkappa} \cos ^{\varkappa-1} \phi\left(B \cos ^{2} \phi+\frac{\chi(\phi)}{\sin ^{2}\left(\omega_{0} / 2\right)}\right), \quad \forall \phi \in\left[-\omega_{0} / 2, \omega_{0} / 2\right],
\end{aligned}
$$

where $\chi(\phi)=\sin \left(\omega_{0} / 2-\phi\right) \cdot \sin \left(\omega_{0} / 2+\phi\right)$. We find that $\chi^{\prime}(\phi)=-\sin 2 \phi$ and $\chi^{\prime}(\phi)=0$ for $\phi=0$. Now we see that $\chi^{\prime \prime}(0)=-2 \cos 0=-2<0$. In this way we have

$$
\max _{\phi \in\left[-\omega_{0} / 2, \omega_{0} / 2\right]} \chi(\phi)=\chi(0)=\sin ^{2}\left(\omega_{0} / 2\right)
$$

and therefore

$$
\begin{aligned}
w\left(x ; y, x^{\prime}\right) & \leq r^{1+\varkappa} \cos ^{\varkappa-1} \phi\left(B \cos ^{2} \phi+1\right) \leq r^{1+\varkappa} \cos ^{\varkappa+1} \phi\left(B+\frac{1}{\cos ^{2} \phi}\right) \\
& \leq r^{1+\varkappa 0}\left(B+\frac{1}{\cos ^{2}\left(\omega_{0} / 2\right)}\right) .
\end{aligned}
$$

Hence (3.4) follows. Finally, (3.5) follows in the same way, in virtue of (3.11).

Now we can estimate $|u(x)|$ for $u$ satisfying $(L)$ in the neighborhood of the conical point.

THEOREM 3.2. Let $u$ be a weak solution of problem $(L)$ and let $u$ satisfy assumptions (a)-(c). Then there exist numbers $d \in(0,1)$ and $\varkappa>0$ depending only on $a_{*}, n, \omega_{0}, f_{0}, h_{0}, g_{0}, \beta_{0}, \gamma_{0}, s$ and the domain $G$ such that

$$
|u(x)-u(0)| \leq C_{0}|x|^{\varkappa+1}, \quad x \in G_{0}^{d},
$$

where the positive constant $C_{0}$ depends only on $a_{*}, n, \omega_{0}, f_{0}, h_{0}, g_{0}, \beta_{0}, \gamma_{0}$, $s, M_{0}$ and the domain $G$, and does not depend on $u$.

Proof. Without loss of generality we may suppose that $u(0) \geq 0$. Consider the barrier function $w$ defined by (3.6) with $\varkappa \in\left(0, \varkappa_{0}\right)$ and the function $v(x)=u(x)-u(0)$. For these, we shall verify Proposition 2.9. Let us calculate the action of $Q$ on these functions. Because of the definition (2.15) and by integration by parts, we have

$$
\begin{aligned}
Q(A w, \eta) \equiv & \int_{G_{0}^{d}}\left\{A a w_{x_{i}} \eta_{x_{i}}+(f(x)+A p w(x)) \eta(x)\right\} d x-\int_{\Omega_{d}} A a \frac{\partial w}{\partial r} \eta(x) d \Omega_{d} \\
& -\int_{\Gamma_{0}^{d}} \alpha(x)\left(g(x)-\frac{1}{r} \gamma(\phi) A w(x)\right) \eta(x) d s \\
& -\int_{\Sigma_{0}^{d}}\left(h(x)-\frac{1}{r} \beta(\phi) A w(x)\right) \eta(x) d s
\end{aligned}
$$




$$
\begin{aligned}
= & \int_{G_{0}^{d}}(f(x)+A p w(x)-A a \Delta w) \eta(x) d x \\
& +\int_{\Gamma_{0}^{d}}(\mathcal{B}[A w]-g(x)) \eta(x) d s+\int_{\Sigma_{0}^{d}}(\mathcal{S}[A w]-h(x)) \eta(x) d s
\end{aligned}
$$

with any $A>0$. Hence, by Lemma 3.1 and conditions (b), (c) we obtain

$$
\begin{aligned}
Q(A w, \eta) \geq & \int_{G_{0}^{d}}\left\{f(x)+A a_{*} m^{2} r^{\varkappa-1}\right\} \eta(x) d x \\
& +\int_{\Gamma_{0}^{d}}\left(A g_{0} r^{\delta}-g(x)\right) \eta(x) d s+\int_{\Sigma_{0}^{d}}\left(A h_{0} r^{\delta}-h(x)\right) \eta(x) d s \\
\geq & \int_{G_{0}^{d}} r^{\varkappa-1}\left\{A a_{*} m^{2}-f_{0} r^{\beta+1-\varkappa}\right\} \eta(x) d x \\
& +g_{0} \int\left(A r^{\delta}-r^{s-1}\right) \eta(x) d s+h_{0} \int_{\Sigma_{0}^{d}}\left(A r^{\delta}-r^{s-1}\right) \eta(x) d s \\
\geq & 0
\end{aligned}
$$

because of $0<\varkappa<\varkappa_{0}$, if $\varkappa_{0}, \delta, A$ are chosen such that

$$
\varkappa_{0} \leq \beta+1, \quad \delta \leq s-1, \quad A \geq \max \left\{1, \frac{f_{0}}{a_{*} m^{2}}\right\} .
$$

On the other hand,

$$
Q(v, \eta) \equiv-\int_{G_{0}^{d}} p u(0) \eta(x) d x-\int_{\Gamma_{0}^{d}} \frac{\gamma(\omega)}{r} u(0) \eta(x) d s-\int_{\Sigma_{0}^{d}} \frac{\sigma(\omega)}{r} u(0) \eta(x) d s \leq 0
$$

and thus

$$
Q(v, \eta) \leq Q(A w, \eta)
$$

for all nonnegative $\eta \in \mathbf{C}^{0}\left(\overline{G_{0}^{d}}\right) \cap \mathbf{W}_{0}^{1}\left(G_{0}^{d}\right)$.

Now we compare $v(x)$ and $w(x)$ on $\Omega_{d}$. Since $x^{2} \geq h^{2} y^{2}$ in $\bar{K}$, from (3.6) we have

$$
\left.w(x)\right|_{r=d} \geq B d^{1+\varkappa} \cos ^{\varkappa+1}\left(\omega_{0} / 2\right) .
$$

On the other hand,

$$
\left.v(x)\right|_{\Omega_{d}}=\left.(u(x)-u(0))\right|_{\Omega_{d}} \leq M_{0}
$$

and therefore from (3.20)-(3.21) we obtain

$$
\begin{aligned}
\left.A w(x)\right|_{\Omega_{d}} & \geq A B d^{1+\varkappa} \cos ^{\varkappa+1}\left(\omega_{0} / 2\right) \geq A B d^{1+\varkappa} \frac{m^{1+\varkappa_{0}}}{\left(1+m^{2}\right)^{\left(1+\varkappa_{0}\right) / 2}} \geq M_{0} \\
& \geq\left. v\right|_{\Omega_{d}},
\end{aligned}
$$


if we choose $A$ possibly greater:

$$
A \geq \frac{M_{0}\left(1+m^{2}\right)^{\left(1+\varkappa_{0}\right) / 2}}{B(m d)^{1+\varkappa_{0}}}
$$

where $B$ satisfies inequalities (3.13) and (3.15).

Finally, if $\Gamma_{0}^{d} \cap \mathcal{D} \neq \emptyset$ and $u(x)=g(x), x \in \Gamma_{0}^{d} \cap \mathcal{D}$, where $|g(x)| \leq g_{0}|x|^{s}$, then

$$
\begin{aligned}
v(x) & =u(x)-u(0) \leq u(x)=g(x) \leq g_{0}|x|^{s}, \quad x \in \Gamma_{0}^{d} \cap \mathcal{D} ; \\
\left.A w\right|_{\Gamma_{0}^{d} \cap \mathcal{D}} & =A B r^{1+\varkappa} \cos ^{\varkappa+1} \frac{\omega_{0}}{2} \geq A B r^{1+\varkappa} \frac{m^{1+\varkappa_{0}}}{\left(1+m^{2}\right)^{\left(1+\varkappa_{0}\right) / 2}} \geq g_{0} r^{s} \\
& \geq\left. v\right|_{\Gamma_{0}^{d} \cap \mathcal{D}},
\end{aligned}
$$

if $\varkappa_{0} \leq s-1$ and if we increase $A$ :

$$
A \geq \frac{\left(1+m^{2}\right)^{\left(1+\varkappa_{0}\right) / 2}}{B m^{1+\varkappa_{0}}} g_{0}
$$

where $B$ satisfies inequalities (3.13) and (3.15). Thus, if we choose large numbers $B>0, A \geq 1$ according to (3.13), (3.15), (3.18), (3.22), (3.23) we ensure the validity of Proposition 2.9.

Therefore, by that proposition,

$$
u(x)-u(0) \leq A w(x), \quad x \in \overline{G_{0}^{d}} .
$$

Similarly, we derive the estimate $u(x)-u(0) \geq-A w(x)$, if we consider an auxiliary function $v(x)=u(0)-u(x)$. Our theorem is thus proved in virtue of (3.4).

Now we will estimate the modulus of the gradient of the solution of problem $(L)$ near a conical point.

THEOREM 3.3. Let u be a weak solution of problem $(L)$, and suppose that assumptions (b)-(c) are satisfied. Let $\varkappa>0$ be defined by Lemma 3.1 with $\varkappa_{0} \leq \beta+1$. Then there exists a number $d>0$ such that

$$
|\nabla u(x)|<C_{1}|x|^{\varkappa}, \quad x \in G_{0}^{d},
$$

where the constant $C_{1}$ does not depend on $u$, but depends only on $a_{*}, a^{*}, n$, $\omega_{0}, f_{0}, h_{0}, g_{0}, \beta_{0}, \gamma_{0}, s, M_{0}$ and the domain $G$.

Proof. Consider the set $G_{\varrho / 2}^{\varrho} \subset G, 0<\varrho<d$. We make the transformation $x=\varrho x^{\prime}, v\left(x^{\prime}\right)=\varrho^{-1-\varkappa} u\left(\varrho x^{\prime}\right)$. The function $v\left(x^{\prime}\right)$ satisfies 
$\left(L^{\prime}\right) \begin{cases}a_{i} \Delta^{\prime} v_{i}+p_{i} \varrho^{2} v_{i}\left(x^{\prime}\right)=\varrho^{1-\varkappa} f_{i}\left(\varrho x^{\prime}\right), & x^{\prime} \in\left(G_{i}\right)_{1 / 2}^{1}, i=1, \ldots, N ; \\ {\left[v\left(x^{\prime}\right)\right]_{\left(\Sigma_{k}\right)_{1 / 2}^{1}}=0,} & k=1, \ldots, N-1 ; \\ {\left[a \frac{\partial v}{\partial n_{k}^{\prime}}\right]_{\left(\Sigma_{k}\right)_{1 / 2}^{1}}+\frac{1}{\left|x^{\prime}\right|} \beta_{k}(\phi) v\left(x^{\prime}\right)=\varrho^{-\varkappa} h_{k}\left(\varrho x^{\prime}\right),} & x^{\prime} \in\left(\Sigma_{k}\right)_{1 / 2}^{1}, \quad k=1, \ldots, N-1 ; \\ \alpha\left(x^{\prime}\right) \cdot a \frac{\partial v}{\partial n^{\prime}}+\frac{1}{\left|x^{\prime}\right|} \gamma(\phi) v\left(x^{\prime}\right)=\varrho^{-\varkappa} g\left(\varrho x^{\prime}\right), \quad x^{\prime} \in \Gamma_{1 / 2}^{1} .\end{cases}$

Now we apply [10, Theorem 16.2, Chapter III] to get

$$
\max _{x^{\prime} \in G_{1 / 2}^{1}}\left|\nabla^{\prime} v\left(x^{\prime}\right)\right| \leq M_{1}^{\prime}
$$

Returning to the variable $x$ and the function $u$ we obtain, from (3.26),

$$
|\nabla u(x)| \leq M_{1} \varrho^{\varkappa}, \quad x \in G_{\varrho / 2}^{\varrho}, 0<\varrho<d .
$$

Putting now $|x|=\frac{2}{3} \varrho$ we obtain the desired estimate (3.25).

Corollary 3.4. Let $u$ be a weak solution of problem $(L)$ and suppose that the assumptions of Theorem 3.3 are satisfied. Then $u(0)=0$ and therefore the inequality (3.16) takes the form

$$
|u(x)| \leq C_{0}|x|^{\varkappa+1}, \quad x \in G_{0}^{d} .
$$

Proof. From the boundary condition it follows that

$$
\gamma(\phi) u(x)=|x| g(x)-\alpha(x) \cdot a|x| \frac{\partial u}{\partial n}, \quad x \in \partial G \backslash \mathcal{O} .
$$

By the assumption (b)-(c) and the estimate (3.25), we obtain

$$
\gamma_{0}|u(x)| \leq \gamma(\phi)|u(x)| \leq|x||g(x)|+a^{*}|x||\nabla u| \leq g_{0}|x|^{s}+C_{1} a^{*}|x|^{\varkappa+1} \text {. }
$$

By letting $|x|$ tend to 0 we deduce, because of the continuity of $u$, that $\gamma_{0}|u(0)| \leq 0$ and taking into account $\gamma_{0}>0$, we find $u(0)=0$.

4. Local estimate at the boundary. We now derive the local boundedness (near the conical point) of a weak solution of $(L)$ in case $\mathcal{D}=\emptyset$.

THEOREM 4.1. Let $u$ be a weak solution of $(L)$. Let assumptions (a)-(c) be satisfied. Suppose, in addition, that $h \in \mathbf{L}_{\infty}\left(\Sigma_{0}\right)$ and $g \in L_{\infty}(\partial G)$. Then

$$
\begin{aligned}
\sup _{G_{0}^{\varkappa \varrho}}|u(x)| \leq & \frac{C}{(1-\varkappa)^{n / t}}\left\{\varrho^{-n / t}\|u\|_{t, G_{0}^{\varrho}}+\varrho^{2(1-n / q)}\|f\|_{q / 2, G_{0}^{\varrho}}\right. \\
& +\varrho\left(\|g\|_{\infty, \Gamma_{0}^{\varrho}}+\|h\|_{\left.\infty, \Sigma_{0}^{\varrho}\right)}\right\}
\end{aligned}
$$

for any $t>0, \varkappa \in(0,1)$ and $\varrho \in(0, d)$, where $C=C\left(n, a_{*}, a^{*}, t, q, G\right)$. 
Proof. We can assume without loss of generality that $u \geq 0$ and $n \geq 3$ (for the general case see $[7, \S 8.6]$ or $[4, \S 1$, Chapter 4$]$ ). We apply the Moser iteration method.

First we assume that $t \geq 2$. We consider the integral identity (II) and make the coordinate transformation $x=\varrho x^{\prime}$. Let $G^{\prime}$ be the image of $G, \partial G^{\prime}$ be the image of $\partial G$, and $\Sigma_{k}^{\prime}$ be the images of $\Sigma_{k}, k=1, \ldots, N-1$. We have $d x=\varrho^{n} d x^{\prime}$ and $d s=\varrho^{n-1} d s^{\prime}$. In addition, we define

$$
\begin{array}{rlrl}
v\left(x^{\prime}\right) & =u\left(\varrho x^{\prime}\right), & & \mathcal{F}\left(x^{\prime}\right)=\varrho^{2} f\left(\varrho x^{\prime}\right), \\
\mathcal{G}\left(x^{\prime}\right)=\varrho g\left(\varrho x^{\prime}\right), & & \mathcal{H}\left(x^{\prime}\right)=\varrho h\left(\varrho x^{\prime}\right) .
\end{array}
$$

Then (II) means

$$
\begin{aligned}
\int_{G^{\prime}}\left\{a v_{x_{j}^{\prime}} \eta_{x_{j}^{\prime}}+p \varrho^{2} v\left(x^{\prime}\right) \eta\left(x^{\prime}\right)\right\} d x^{\prime}+\varrho \int_{\Sigma^{\prime}} \frac{1}{\left|x^{\prime}\right|} \beta(\phi) v\left(x^{\prime}\right) \eta\left(x^{\prime}\right) d s^{\prime} \\
+\varrho \int_{\partial G^{\prime}} \frac{1}{\left|x^{\prime}\right|} \gamma(\phi) v\left(x^{\prime}\right) \eta\left(x^{\prime}\right) d s^{\prime} \\
=\int_{\partial G^{\prime}} \mathcal{G}\left(x^{\prime}\right) \eta\left(x^{\prime}\right) d s^{\prime}+\int_{\Sigma_{0}^{\prime}} \mathcal{H}\left(x^{\prime}\right) \eta\left(x^{\prime}\right) d s^{\prime}-\int_{G^{\prime}} \mathcal{F}\left(x^{\prime}\right) \eta\left(x^{\prime}\right) d x^{\prime}
\end{aligned}
$$

for all $\eta \in \mathbf{C}^{0}\left(\overline{G^{\prime}}\right) \cap \mathbf{W}_{0}^{1}\left(G^{\prime}\right)$. Now define

$$
k=k(\varrho)=a_{*}^{-1}\left(\|\mathcal{F}\|_{q / 2, G_{0}^{1}}+\|\mathcal{G}\|_{\infty, \Gamma_{0}^{1}}+\|\mathcal{H}\|_{\infty, \Sigma_{0}^{1}}\right)
$$

and set

$$
\bar{v}\left(x^{\prime}\right)=v\left(x^{\prime}\right)+k .
$$

Observe that

$$
\begin{aligned}
|\mathcal{F}| \bar{v} & =\frac{1}{k}|\mathcal{F}| \cdot k \bar{v}=\frac{1}{k}|\mathcal{F}|(\bar{v}-v) \cdot \bar{v} \\
& =\frac{1}{k}|\mathcal{F}| \cdot \bar{v}^{2}-\frac{1}{k}|\mathcal{F}| \cdot v \bar{v} \leq \frac{1}{k}|\mathcal{F}| \cdot \bar{v}^{2} ; \\
|\mathcal{H}| \bar{v} & \leq \frac{1}{k}|\mathcal{H}| \cdot \bar{v}^{2} ; \quad|\mathcal{G}| \bar{v} \leq \frac{1}{k}|\mathcal{G}| \cdot \bar{v}^{2}
\end{aligned}
$$

in the same way. As a test function in the integral identity (II) ${ }^{\prime}$ we choose

$$
\eta\left(x^{\prime}\right)=\zeta^{2}\left(\left|x^{\prime}\right|\right) \bar{v}^{t-1}\left(x^{\prime}\right),
$$

where $\zeta\left(\left|x^{\prime}\right|\right) \in \mathbf{C}_{0}^{\infty}([0,1])$ is a nonnegative function to be further specified. By the chain and product rules, $v$ is indeed a test function in (II) ${ }^{\prime}$ and also

$$
\eta_{x_{i}^{\prime}}=(t-1) \bar{v}^{t-2} v_{x_{i}^{\prime}} \zeta^{2}\left(\left|x^{\prime}\right|\right)+2 \zeta \zeta_{x_{i}^{\prime}} \bar{v}^{t-1}\left(x^{\prime}\right),
$$


so that by substitution into (II $)^{\prime}$, since $p \geq 0$ we obtain

$$
\begin{aligned}
(t-1) \int_{G_{0}^{1}} a\left|\nabla^{\prime} v\right|^{2} \bar{v}^{t-2} \zeta^{2}\left(\left|x^{\prime}\right|\right) d x^{\prime}+\varrho \int_{\Sigma_{0}^{1}} \frac{1}{\left|x^{\prime}\right|} \beta(\phi)\left(\bar{v}\left(x^{\prime}\right)-k\right) \bar{v}^{t-1}\left(x^{\prime}\right) \zeta^{2}\left(\left|x^{\prime}\right|\right) d s^{\prime} \\
\quad+\varrho \int_{\Gamma_{0}^{1}} \frac{1}{\left|x^{\prime}\right|} \gamma(\phi)\left(\bar{v}\left(x^{\prime}\right)-k\right) \bar{v}^{t-1}\left(x^{\prime}\right) \zeta^{2}\left(\left|x^{\prime}\right|\right) d s^{\prime} \\
\leq-2 \int_{G_{0}^{1}} a\left(\varrho x^{\prime}\right) \zeta_{x_{j}^{\prime}} v_{x_{j}^{\prime}} \bar{v}^{t-1} \zeta\left(\left|x^{\prime}\right|\right) d x^{\prime}+\int_{\Gamma_{0}^{1}} \mathcal{G}\left(x^{\prime}\right) \bar{v}^{t-1}\left(x^{\prime}\right) \zeta^{2}\left(\left|x^{\prime}\right|\right) d s^{\prime} \\
\quad+\int_{\Sigma_{0}^{1}} \mathcal{H}\left(x^{\prime}\right) \bar{v}^{t-1}\left(x^{\prime}\right) \zeta^{2}\left(\left|x^{\prime}\right|\right) d s^{\prime}-\int_{G_{0}^{1}} \mathcal{F}\left(x^{\prime}\right) \bar{v}^{t-1}\left(x^{\prime}\right) \zeta^{2}\left(x^{\prime}\right) d x^{\prime} .
\end{aligned}
$$

By (c) and (4.5), it follows that

$$
\begin{aligned}
& (t-1) \int_{G_{0}^{1}} a\left|\nabla^{\prime} v\right|^{2} \cdot \bar{v}^{t-2} \zeta^{2}\left(\left|x^{\prime}\right|\right) d x^{\prime} \\
\leq & \int_{G_{0}^{1}}\left(2 a^{*}\left|\nabla^{\prime} v\right| \cdot\left|\nabla^{\prime} \zeta\right| \cdot \bar{v}^{t-1} \zeta\left(\left|x^{\prime}\right|\right)+\frac{1}{k}\left|\mathcal{F}\left(x^{\prime}\right)\right| \cdot \bar{v}^{t} \zeta^{2}\left(\left|x^{\prime}\right|\right)\right) d x^{\prime} \\
+ & \frac{1}{k}\|\mathcal{G}\|_{\infty, \Gamma_{0}^{1}} \cdot \int_{\Gamma_{0}^{1}} \bar{v}^{t}\left(x^{\prime}\right) \zeta^{2}\left(\left|x^{\prime}\right|\right) d s^{\prime}+\frac{1}{k}\|\mathcal{H}\|_{\infty, \Sigma_{0}^{1}} \cdot \int_{\Sigma_{0}^{1}} \bar{v}^{t}\left(x^{\prime}\right) \zeta^{2}\left(\left|x^{\prime}\right|\right) d s^{\prime} .
\end{aligned}
$$

By the Cauchy inequality, for any $\varepsilon>0$,

$$
\begin{aligned}
2 a^{*}\left|\nabla^{\prime} v\right| \cdot\left|\nabla^{\prime} \zeta\right| \cdot \bar{v}^{t-1} \zeta & \leq 2\left(\sqrt{a}\left|\nabla^{\prime} v\right| \cdot \bar{v}^{t / 2-1} \zeta\right) \cdot\left(\frac{a^{*}}{\sqrt{a_{*}}} \bar{v}^{t / 2}\left|\nabla^{\prime} \zeta\right|\right) \\
& \leq \varepsilon a\left|\nabla^{\prime} v\right|^{2} \cdot \bar{v}^{t-2} \zeta^{2}+\frac{\left(a^{*}\right)^{2}}{\varepsilon a_{*}} \bar{v}^{t}\left|\nabla^{\prime} \zeta\right|^{2} .
\end{aligned}
$$

To estimate the boundary integrals we apply the inequality (2.10). Then from (4.6) it follows that

$$
\begin{aligned}
& \quad(t-1) \int_{G_{0}^{1}} a\left|\nabla^{\prime} v\right|^{2} \cdot \bar{v}^{t-2} \zeta^{2}\left(\left|x^{\prime}\right|\right) d x^{\prime} \leq \varepsilon \int_{G_{0}^{1}} a\left|\nabla^{\prime} v\right|^{2} \cdot \bar{v}^{t-2} \zeta^{2}\left(\left|x^{\prime}\right|\right) d x^{\prime} \\
& +\int_{G_{0}^{1}}\left(\frac{\left(a^{*}\right)^{2}}{\varepsilon a_{*}} \bar{v}^{t} \cdot\left|\nabla^{\prime} \zeta\right|^{2}+\frac{1}{k}\left|\mathcal{F}\left(x^{\prime}\right)\right| \cdot \bar{v}^{t} \zeta^{2}\left(\left|x^{\prime}\right|\right)\right) d x^{\prime} \\
& +\frac{1}{k}\left(\|\mathcal{G}\|_{\infty, \Gamma_{0}^{1}}+\|\mathcal{H}\|_{\infty, \Sigma_{0}^{1}}\right) \cdot \int_{G_{0}^{1}}\left(\delta\left|\nabla^{\prime}\left(\zeta \bar{v}^{t / 2}\right)\right|^{2}+\frac{1}{\delta} c_{0} \bar{v}^{t} \zeta^{2}\right) d x^{\prime}, \quad \forall \varepsilon, \delta>0 .
\end{aligned}
$$


From

$$
\begin{aligned}
& \left|\nabla^{\prime}\left(\zeta \bar{v}^{t / 2}\right)\right|^{2} \leq 2\left(\zeta^{2}\left|\nabla^{\prime}\left(\bar{v}^{t / 2}\right)\right|^{2}+\bar{v}^{t}\left|\nabla^{\prime} \zeta\right|^{2}\right), \\
& \left|\nabla^{\prime}\left(\bar{v}^{t / 2}\right)\right|^{2}=\frac{t^{2}}{4} \bar{v}^{t-2}\left|\nabla^{\prime} v\right|^{2}
\end{aligned}
$$

it follows that

$$
\left|\nabla^{\prime}\left(\zeta \bar{v}^{t / 2}\right)\right|^{2} \leq \frac{t^{2}}{2} \bar{v}^{t-2}\left|\nabla^{\prime} v\right|^{2} \zeta^{2}+2 \bar{v}^{t}\left|\nabla^{\prime} \zeta\right|^{2} .
$$

Now, by (4.7), (4.9), choosing $\varepsilon=\frac{t-1}{2}$ we find that

$$
\begin{aligned}
& \text { (4.10) } \quad \frac{t-1}{2} \int_{G_{0}^{1}} a\left|\nabla^{\prime} v\right|^{2} \cdot \bar{v}^{t-2} \zeta^{2}\left(\left|x^{\prime}\right|\right) d x^{\prime} \\
& \leq \frac{\delta t^{2}}{2 a_{*}} \cdot \frac{\|\mathcal{G}\|_{\infty, \Gamma_{0}^{1}}+\|\mathcal{H}\|_{\infty, \Sigma_{0}^{1}}}{k} \cdot \int_{G_{0}^{1}} a\left|\nabla^{\prime} v\right|^{2} \cdot \bar{v}^{t-2} \zeta^{2}\left(\left|x^{\prime}\right|\right) d x^{\prime} \\
& \quad+\frac{1}{k} \int_{G_{0}^{1}}\left\{\left|\mathcal{F}\left(x^{\prime}\right)\right|+\frac{c_{0}}{\delta}\left(\|\mathcal{G}\|_{\infty, \Gamma_{0}^{1}}+\|\mathcal{H}\|_{\infty, \Sigma_{0}^{1}}\right)\right\} \cdot \bar{v}^{t} \zeta^{2}\left(\left|x^{\prime}\right|\right) d x^{\prime} \\
& \quad+\int_{G_{0}^{1}}\left\{\frac{2\left(a^{*}\right)^{2}}{(t-1) a_{*}}+\frac{2 \delta}{k}\left(\|\mathcal{G}\|_{\infty, \Gamma_{0}^{1}}+\|\mathcal{H}\|_{\infty, \Sigma_{0}^{1}}\right)\right\} \bar{v}^{t} \cdot\left|\nabla^{\prime} \zeta\right|^{2} d x^{\prime}, \quad \forall \delta \in(0,1] .
\end{aligned}
$$

We now choose $\delta=\frac{t-1}{2 t^{2}}$; by the definition of $k$ in (4.3) the last inequality (4.10) may be rewritten as

$$
\begin{aligned}
\int_{G_{0}^{1}} a\left|\nabla^{\prime} v\right|^{2} \cdot \bar{v}^{t-2} \zeta^{2}\left(\left|x^{\prime}\right|\right) d x^{\prime} \leq & \left(\frac{8\left(a^{*}\right)^{2}}{a_{*}(t-1)^{2}}+\frac{4 a_{*}}{t^{2}}\right) \cdot \int_{G_{0}^{1}}\left|\nabla^{\prime} \zeta\right|^{2} \bar{v}^{t}\left(x^{\prime}\right) d x^{\prime} \\
& +\frac{4}{t-1} \int_{G_{0}^{1}}\left(\frac{2 a_{*} c_{0} t^{2}}{t-1}+\frac{\left|\mathcal{F}\left(x^{\prime}\right)\right|}{k}\right) \cdot \bar{v}^{t} \zeta^{2}\left(\left|x^{\prime}\right|\right) d x^{\prime}
\end{aligned}
$$

But, by (4.8), this means that

$$
\begin{array}{r}
\int_{G_{0}^{1}} a\left|\nabla^{\prime}\left(\bar{v}^{t / 2}\right)\right|^{2} \zeta^{2}\left(\left|x^{\prime}\right|\right) d x^{\prime} \leq\left(\frac{2 t^{2}\left(a^{*}\right)^{2}}{a_{*}(t-1)^{2}}+a_{*}\right) \cdot \int_{G_{0}^{1}}\left|\nabla^{\prime} \zeta\right|^{2} \bar{v}^{t}\left(x^{\prime}\right) d x^{\prime} \\
+\frac{t^{2}}{t-1} \int_{G_{0}^{1}}\left(\frac{2 a_{*} c_{0} t^{2}}{t-1}+\frac{\left|\mathcal{F}\left(x^{\prime}\right)\right|}{k}\right) \cdot \bar{v}^{t} \zeta^{2}\left(\left|x^{\prime}\right|\right) d x^{\prime}
\end{array}
$$

Further, as $t \geq 2$ we have 


$$
\begin{aligned}
2 c_{0} a_{*} \frac{t^{2}}{(t-1)^{2}} & =2 c_{0} a_{*}\left(1+\frac{2}{t-1}+\frac{1}{(t-1)^{2}}\right) \leq 8 c_{0} a_{*} ; \\
\frac{2\left(a^{*}\right)^{2} t^{2}}{a_{*}(t-1)^{2}} & \leq \frac{8\left(a^{*}\right)^{2}}{a_{*}} .
\end{aligned}
$$

Therefore the inequality (4.11) can be rewritten as

$$
\begin{aligned}
\int_{G_{0}^{1}} a\left|\nabla^{\prime}\left(\bar{v}^{t / 2}\right)\right|^{2} \zeta^{2}\left(\left|x^{\prime}\right|\right) d x^{\prime} \leq & C_{1}(1+t)^{2} \int_{G_{0}^{1}} a\left(\left|\nabla^{\prime} \zeta\right|^{2}+\zeta^{2}\left(\left|x^{\prime}\right|\right)\right) \bar{v}^{t} d x^{\prime} \\
& +C_{2}(1+t)^{2} \int_{G_{0}^{1}} \frac{\left|\mathcal{F}\left(x^{\prime}\right)\right|}{k} \cdot a \bar{v}^{t} \zeta^{2}\left(\left|x^{\prime}\right|\right) d x^{\prime},
\end{aligned}
$$

where the constants $C_{1}, C_{2}$ depend only on $c_{0}, a_{*}, a^{*}$ and are independent of $t$. Setting

$$
w\left(x^{\prime}\right)=\sqrt{a} \cdot \bar{v}^{t / 2}\left(x^{\prime}\right)
$$

from (4.12) we obtain

$$
\begin{aligned}
\int_{G_{0}^{1}}\left|\nabla^{\prime} w\right|^{2} \zeta^{2}\left(\left|x^{\prime}\right|\right) d x^{\prime} \leq & C_{1}(1+t)^{2} \int_{G_{0}^{1}}\left(\left|\nabla^{\prime} \zeta\right|^{2}+\zeta^{2}\left(\left|x^{\prime}\right|\right)\right) w^{2}\left(x^{\prime}\right) d x^{\prime} \\
& +C_{2}(1+t)^{2} \int_{G_{0}^{1}} \frac{\left|\mathcal{F}\left(x^{\prime}\right)\right|}{k} \cdot w^{2}\left(x^{\prime}\right) \zeta^{2}\left(\left|x^{\prime}\right|\right) d x^{\prime} .
\end{aligned}
$$

The desired iteration process can now be continued from (4.14). By the Sobolev imbedding theorem we have

$$
\|\zeta w\|_{\frac{2 n}{n-2}, G_{0}^{1}}^{2} \leq C^{*} \int_{G_{0}^{1}}\left(\left|\nabla^{\prime} \zeta\right|^{2} w^{2}\left(x^{\prime}\right)+\zeta^{2}\left(\left|x^{\prime}\right|\right)\left|\nabla^{\prime} w\right|^{2}\right) d x^{\prime}, \quad n \geq 3,
$$

where $C^{*}$ depends only on $n$ and the domain $G$. Using the Hölder inequality

$$
\int_{G_{0}^{1}}\left|F\left(x^{\prime}\right)\right| \cdot w^{2}\left(x^{\prime}\right) \zeta^{2}\left(x^{\prime}\right) d x^{\prime} \leq\|F\|_{q / 2, G_{0}^{1}} \cdot\|w \zeta\|_{\frac{2 q}{q-2}, G_{0}^{1}}^{2}, \quad q>2,
$$

we get from $(4.14)-(4.16)$

$$
\begin{aligned}
\|\zeta w\|_{\frac{2 n}{n-2}, G_{0}^{1}}^{2} \leq & C_{3}(1+t)^{2} \int_{G_{0}^{1}}\left(\left|\nabla^{\prime} \zeta\right|^{2}+\zeta^{2}\left(\left|x^{\prime}\right|\right)\right) w^{2}\left(x^{\prime}\right) d x^{\prime} \\
& +C_{4}(1+t)^{2}\left\|\frac{\left|\mathcal{F}\left(x^{\prime}\right)\right|}{k}\right\|_{q / 2, G_{0}^{1}} \cdot\|w \zeta\|_{\frac{2 q}{q-2}, G_{0}^{1}}^{2}, \quad q>n .
\end{aligned}
$$

By the interpolation inequality for $L_{q}$ norms,

$$
\|\zeta w\|_{\frac{2 q}{q-2}, G_{0}^{1}} \leq \varepsilon\|\zeta w\|_{\frac{2 n}{n-2}, G_{0}^{1}}+\varepsilon^{\frac{n}{n-q}}\|\zeta w\|_{2, G_{0}^{1}}, \quad q>n, \forall \varepsilon>0,
$$

from (4.17)-(4.18) it follows that 


$$
\begin{aligned}
\|\zeta w\|_{\frac{2 n}{n-2}, G_{0}^{1}} \leq & (1+t) \sqrt{C_{3}} \cdot\left\|\left(\zeta+\left|\nabla^{\prime} \zeta\right|\right) w\right\|_{2, G_{0}^{1}} \\
& +(1+t) \sqrt{C_{4}}\left\|\frac{\left|\mathcal{F}\left(x^{\prime}\right)\right|}{k}\right\|_{q / 2, G_{0}^{1}}^{1 / 2} \\
& \times\left(\varepsilon\|w \zeta\|_{\frac{2 n}{n-2}, G_{0}^{1}}^{2}+\varepsilon^{\frac{n}{n-q}}\|\zeta w\|_{2, G_{0}^{1}}\right), \quad q>n, \forall \varepsilon>0 .
\end{aligned}
$$

Choosing $\varepsilon=\frac{1}{2 \sqrt{C_{4}}}(1+t)^{-1}\left\|\frac{\left|\mathcal{F}\left(x^{\prime}\right)\right|}{k}\right\|_{q / 2, G_{0}^{1}}^{-1 / 2}$ we obtain

$$
\|\zeta w\|_{\frac{2 n}{n-2}, G_{0}^{1}} \leq C(1+t)^{\frac{q}{q-n}}\left\|\left(\zeta+\left|\nabla^{\prime} \zeta\right|\right) w\right\|_{2, G_{0}^{1}}, \quad q>n \geq 3,
$$

where $C$ depends only on $c_{0}, n, a_{*}, a^{*}, q, \operatorname{diam} G$, and is independent of $t$. Recalling the definition (4.13) of $w$ and taking into account that $t \geq 2$ from (4.20) we finally establish the inequality

$$
\left\|\zeta \cdot \bar{v}^{t / 2}\right\|_{\frac{2 n}{n-2}, G_{0}^{1}} \leq C t^{\frac{q}{q-n}}\left\|\left(\zeta+\left|\nabla^{\prime} \zeta\right|\right) \cdot \bar{v}^{t / 2}\right\|_{2, G_{0}^{1}}, \quad q>n \geq 3 .
$$

This inequality can now be iterated to yield the desired estimate.

For all $\varkappa \in(0,1)$ we define $G_{(j)}^{\prime} \equiv G_{0}^{\varkappa+(1-\varkappa) 2^{-j}}, j=0,1,2, \ldots$ It is easy to verify that $G_{0}^{\varkappa} \equiv G_{(\infty)}^{\prime} \subset \cdots \subset G_{(j+1)}^{\prime} \subset G_{(j)}^{\prime} \subset \cdots \subset G_{(0)}^{\prime} \equiv G_{0}^{1}$. Now we consider a sequence of cut-off functions $\zeta_{j} \in \mathbf{C}^{\infty}\left(G_{(j)}^{\prime}\right)$ such that

$$
\begin{gathered}
0 \leq \zeta_{j}\left(x^{\prime}\right) \leq 1, \quad \zeta_{j}\left(x^{\prime}\right) \equiv 1 \text { in } G_{(j+1)}^{\prime}, \quad \zeta_{j}\left(x^{\prime}\right) \equiv 0 \text { for }\left|x^{\prime}\right|>\varkappa+2^{-j}(1-\varkappa) ; \\
\left|\nabla^{\prime} \zeta_{j}\right| \leq \frac{2^{j+1}}{1-\varkappa} \quad \text { for } \varkappa+2^{-j-1}(1-\varkappa)<\left|x^{\prime}\right|<\varkappa+2^{-j}(1-\varkappa) .
\end{gathered}
$$

We also define the number sequence $t_{j}=t\left(\frac{n}{n-2}\right)^{j}, j=0,1,2, \ldots$ Now we rewrite the inequality (4.21) replacing $\zeta\left(\left|x^{\prime}\right|\right)$ by $\zeta_{j}\left(x^{\prime}\right)$ and $t$ by $t_{j}$; then taking the $t_{j}$ th roots, we obtain

$$
\|\bar{v}\|_{t_{j+1}, G_{(j+1)}^{\prime}} \leq\left(\frac{C}{1-\varkappa}\right)^{2 / t_{j}} \cdot 4^{j / t_{j}} \cdot\left(t_{j}\right)^{\frac{2 q}{q-n} \cdot \frac{1}{t_{j}}}\|\bar{v}\|_{t_{j}, G_{(j)}^{\prime}} .
$$

After iteration, we find that

$$
\begin{aligned}
\|\bar{v}\|_{t_{j+1}, G_{(j+1)}^{\prime}} \leq & \left\{\frac{C t^{\frac{1}{q-1}}}{1-\varkappa} \cdot\left(\frac{n}{n-2}\right)^{\frac{q}{q-n}}\right\}^{2 \sum_{j=0}^{\infty} 1 / t_{j}} \\
& \times 4^{\sum_{j=0}^{\infty} j / t_{j}} \cdot\|\bar{v}\|_{t, G_{0}^{1}} .
\end{aligned}
$$

Notice that the series $\sum_{j=0}^{\infty} j / t_{j}$ is convergent by the d'Alembert ratio test, and $\sum_{j=0}^{\infty} 1 / t_{j}=n / 2 t$ is a geometric series. Therefore from (4.22) we get

$$
\|\bar{v}\|_{t_{j+1}, G_{(j+1)}^{\prime}} \leq \frac{C}{(1-\varkappa)^{n / t}}\|\bar{v}\|_{t, G_{0}^{1}} .
$$


Consequently, letting $j \rightarrow \infty$, we have

$$
\sup _{x^{\prime} \in G_{0}^{\varkappa}}\left|\bar{v}\left(x^{\prime}\right)\right| \leq \frac{C}{(1-\varkappa)^{n / t}}\|\bar{v}\|_{t, G_{0}^{1}}
$$

Hence, by the definition (4.4) of $\bar{v}\left(x^{\prime}\right)$ and (4.3) of $k$, we obtain

$$
\sup _{x^{\prime} \in G_{0}^{\varkappa}} v\left(x^{\prime}\right) \leq \frac{C}{(1-\varkappa)^{n / t}}\left(\|v\|_{t, G_{0}^{1}}+\|\mathcal{F}\|_{q / 2, G_{0}^{1}}+\|\mathcal{G}\|_{\infty, \Gamma_{0}^{1}}+\|\mathcal{H}\|_{\infty, \Sigma_{0}^{1}}\right) .
$$

Returning to the variables $x, u$ via (4.2) and considering the similar inequality with $u$ replaced by $-u$, we obtain the required estimate (4.1) in the case $t \geq 2$.

Let now $0<t<2$. We consider (4.1) with $t=2$ :

$$
\sup _{x \in G_{0}^{\varkappa \varrho}}|u(x)| \leq \frac{C}{[(1-\varkappa) \varrho]^{n / 2}}\|u\|_{2, G_{0}^{\varrho}}+K(\varrho)
$$

where

$$
K(\varrho)=\frac{C}{(1-\varkappa)^{n / 2}}\left\{\varrho^{2(1-n / q)}\|f\|_{q / 2, G_{0}^{\varrho}}+\varrho\left(\|g\|_{\infty, \Gamma_{0}^{\varrho}}+\|h\|_{\infty, \Sigma_{0}^{\varrho}}\right)\right\} .
$$

Now, using the Young inequality with $s=\frac{2}{t}$ and $s^{\prime}=\frac{2}{2-t}$ we can write

$$
\begin{aligned}
\frac{C}{[(1-\varkappa) \varrho]^{n / 2}}\|u\|_{2, G_{0}^{\varrho}} & =\frac{C}{[(1-\varkappa) \varrho]^{n / 2}}\left(\int_{G_{0}^{\varrho}} u^{t} \cdot u^{2-t} d x\right)^{1 / 2} \\
& \left.\leq \sup _{G_{0}^{\varrho}}|u(x)|\right)^{1-t / 2} \cdot \frac{C}{[(1-\varkappa) \varrho]^{n / 2}}\|u\|_{t, G_{0}^{\varrho}}^{t / 2} \\
& \leq \frac{1}{2} \sup _{G_{0}^{\varrho}}|u(x)|+\frac{C_{1}}{[(1-\varkappa) \varrho]^{n / t}}\|u\|_{t, G_{0}^{\varrho}}
\end{aligned}
$$

Define $\psi(s)=\sup _{x \in G_{0}^{s}}|u(x)|$. Then from (4.23)-(4.24) it follows that

$$
\psi(\varkappa \varrho) \leq \frac{1}{2} \psi(\varrho)+\frac{C_{1}}{[(1-\varkappa) \varrho]^{n / t}}\|u\|_{t, G_{0}^{\varrho}}+K(\varrho), \quad \varkappa \in(0,1) .
$$

Further we apply the following statement:

Proposition 4.2 (see [4, Lemma 4.1 in Chapter 2]). Let $\psi$ be a bounded nonnegative function defined on $[0, \varrho]$. Suppose that

$$
\psi(\sigma) \leq \delta \psi(s)+\frac{A}{(s-\sigma)^{\alpha}}+B
$$

for any $0 \leq \sigma<s \leq \varrho$, where $\delta \in(0,1)$, and $A, B$ and $\alpha$ are nonnegative constants. Then

$$
\psi(r) \leq C\left[\frac{A}{(R-r)^{\alpha}}+B\right], \quad 0 \leq r<R \leq \varrho,
$$

where $C$ depends only on $\alpha, \delta$. 
By this proposition, letting $r=\varkappa \varrho, R=\varrho, \delta=1 / 2, \alpha=n / t, A=$ $C_{1}\|u\|_{t, G_{0}^{\varrho}}, B=K(\varrho)$ from (4.25) we obtain the validity of the required estimate (4.1) in the case $0<t<2$.

The proof of Theorem 4.1 is complete.

5. Global integral estimates. First we will obtain a global estimate for the Dirichlet integral.

THEOREM 5.1. Let $u$ be a weak solution of problem $(L)$. Let assumptions (a) - (c) be satisfied. Suppose, in addition, that $\mathcal{D}=\emptyset$. Then

$$
\begin{aligned}
\int_{G} a|\nabla u|^{2} d x+\int_{\Sigma} \frac{\beta(\phi)}{r} u^{2}(x) d s+\int_{\partial G} \frac{\gamma(\phi)}{r} u^{2}(x) d s \\
\leq C\left\{\int_{G}\left(u^{2}(x)+f^{2}(x)\right) d x+\frac{1}{\beta_{0}} \int_{\Sigma} r h^{2}(x) d s+\frac{1}{\gamma_{0}} \int_{\partial G} r g^{2}(x) d s\right\},
\end{aligned}
$$

where the constant $C$ depends only on $p^{*}$.

Proof. Setting in $(I I) \eta(x)=u(x)$ and using the classical Hölder inequality, by assumptions (a), (c), we get

$$
\begin{aligned}
& \int_{G} a|\nabla u|^{2} d x+\int_{\Sigma} \frac{\beta(\phi)}{r} u^{2}(x) d s+\int_{\partial G} \frac{\gamma(\phi)}{r} u^{2}(x) d s \\
& \leq p^{*} \int_{G} u^{2}(x) d x+\int_{\Sigma}|u||h(x)| d s+\int_{\partial G}|u||g(x)| d s+\int_{\partial G}|u||f(x)| d x .
\end{aligned}
$$

By the Cauchy inequality, in view of (c), we obtain

$$
\begin{aligned}
\int_{\Sigma}|u||h(x)| d s & =\int_{\Sigma}\left(\sqrt{\frac{\beta(\phi)}{r}}|u|\right)\left(\sqrt{\frac{r}{\beta(\phi)}}|h(x)|\right) d s \\
& \leq \frac{1}{2} \int_{\Sigma} \frac{\beta(\phi)}{r} u^{2}(x) d s+\frac{1}{2 \beta_{0}} \int_{\Sigma} r h^{2}(x) d s ; \\
\int_{\partial G}|u||g(x)| d s & =\int_{\partial G}\left(\sqrt{\frac{\gamma(\phi)}{r}}|u|\right)\left(\sqrt{\frac{r}{\gamma(\phi)}}|g(x)|\right) d s \\
& \leq \frac{1}{2} \int_{\partial G} \frac{\gamma(\phi)}{r} u^{2}(x) d s+\frac{1}{2 \gamma_{0}} \int_{\partial G} r g^{2}(x) d s ; \\
\int_{\partial G}|u||f(x)| d x & \leq \frac{1}{2} \int_{\partial G}|u|^{2} d x+\frac{1}{2} \int_{\partial G}|f|^{2} d x .
\end{aligned}
$$

Hence we get the desired inequality (5.1). 
Further, we will obtain a global estimate for the weighted Dirichlet integral.

THEOREM 5.2. Let $u$ be a weak solution of problem $(L)$ and let $\lambda$ be as in (1.3), and $\varkappa_{0}$ be as in Lemma 3.1. Let assumptions (a)-(c) be satisfied. Suppose, in addition, that $\lambda>1$ and $0<\varkappa \leq \min \left(s-1, \varkappa_{0}, \lambda-1\right)$ as well as

$$
f \in \stackrel{\mathbf{W}}{\alpha}_{\alpha}^{0}(G), \quad \int_{\Sigma} r^{\alpha-1} h^{2}(x) d s<\infty, \quad \int_{\partial G} r^{\alpha-1} g^{2}(x) d s<\infty,
$$

where

$$
2-n-2 \varkappa<\alpha \leq 2
$$

Then $u \in \stackrel{\circ}{\mathbf{W}}_{\alpha-2}^{1}(G)$ and

$$
\begin{aligned}
& \int_{G} a\left(r^{\alpha-2}|\nabla u|^{2}+r^{\alpha-4} u^{2}\right) d x+\int_{\Sigma} r^{\alpha-3} \beta(\phi) u^{2}(x) d s \\
&+\int_{\partial G} \alpha(x) r^{\alpha-3} \gamma(\phi) u^{2}(x) d s \\
& \leq C\left\{\int_{G}\left(u^{2}+\left(1+r^{\alpha}\right) f^{2}(x)\right) d x+\int_{\Sigma} r^{\alpha-1} h^{2}(x) d s+\int_{\partial G} \alpha(x) r^{\alpha-1} g^{2}(x) d s\right\},
\end{aligned}
$$

where the constant $C>0$ depends only on $q, n, a_{*}, a^{*}, \alpha, \lambda$ and the domain $G$.

Proof. Setting in (II) $\eta(x)=r_{\varepsilon}^{\alpha-2} u(x)$, since $\eta_{x_{i}}=r_{\varepsilon}^{\alpha-2} u_{x_{i}}+(\alpha-2)$ $\cdot r_{\varepsilon}^{\alpha-3} \frac{x_{i}-\varepsilon l_{i}}{r_{\varepsilon}} u(x)$ we obtain

$$
\begin{aligned}
\int_{G} a r_{\varepsilon}^{\alpha-2}|\nabla u|^{2} d x+\int_{\Sigma} r^{-1} r_{\varepsilon}^{\alpha-2} \beta(\phi) u^{2}(x) d s+\int_{\partial G} \alpha(x) r^{-1} r_{\varepsilon}^{\alpha-2} \gamma(\phi) u^{2}(x) d s \\
=\frac{2-\alpha}{2} \int_{G} a r_{\varepsilon}^{\alpha-4}\left(x_{j}-\varepsilon l_{j}\right)\left(u^{2}\right)_{x_{j}} d x-\int_{G}(p u(x)+f(x)) r_{\varepsilon}^{\alpha-2} u(x) d x \\
\quad+\int_{\Sigma} r_{\varepsilon}^{\alpha-2} u(x) h(x) d s+\int_{\partial G} \alpha(x) r_{\varepsilon}^{\alpha-2} u(x) g(x) d s .
\end{aligned}
$$

We transform the first integral on the right by integrating by parts:

$$
\int_{G} a r_{\varepsilon}^{\alpha-4}\left(x_{j}-\varepsilon l_{j}\right) \frac{\partial u^{2}}{\partial x_{j}} d x=\sum_{i=1}^{N} \int_{G_{i}} a_{i} r_{\varepsilon}^{\alpha-4}\left(x_{j}-\varepsilon l_{j}\right) \frac{\partial u_{i}^{2}}{\partial x_{j}} d x
$$

$$
=-\int_{G} a u^{2} \frac{\partial}{\partial x_{i}}\left(r_{\varepsilon}^{\alpha-4}\left(x_{i}-\varepsilon l_{i}\right)\right) d x+\sum_{i=1}^{N} \int_{\partial G_{i}} a_{i} u_{i}^{2} r_{\varepsilon}^{\alpha-4}\left(x_{j}-\varepsilon l_{j}\right) \cos \left(\vec{n}, x_{j}\right) d s
$$




$$
\begin{aligned}
= & -\int_{G} a u^{2} \frac{\partial}{\partial x_{i}}\left(r_{\varepsilon}^{\alpha-4}\left(x_{i}-\varepsilon l_{i}\right)\right) d x+\int_{\partial G} a u^{2} r_{\varepsilon}^{\alpha-4}\left(x_{i}-\varepsilon l_{i}\right) \cos \left(\vec{n}, x_{i}\right) d s \\
& +\sum_{k=1}^{N-1}[a]_{\Sigma_{k}} \int_{\Sigma_{k}} u^{2} r_{\varepsilon}^{\alpha-4}\left(x_{i}-\varepsilon l_{i}\right) \cos \left(\vec{n}_{k}, x_{i}\right) d s,
\end{aligned}
$$

because of $[u]_{\Sigma_{k}}=0, k=1, \ldots, N-1$. By elementary calculation we have:

(1) $\frac{\partial}{\partial x_{i}}\left(r_{\varepsilon}^{\alpha-4}\left(x_{i}-\varepsilon l_{i}\right)\right)=n r_{\varepsilon}^{\alpha-4}+(\alpha-4)\left(x_{i}-\varepsilon l_{i}\right) r_{\varepsilon}^{\alpha-5} \frac{x_{i}-\varepsilon l_{i}}{r_{\varepsilon}}=$ $(n+\alpha-4) r_{\varepsilon}^{\alpha-4}$

(2) by $(2.1),\left.\left(x_{i}-\varepsilon l_{i}\right) \cos \left(\vec{n}_{k}, x_{i}\right)\right|_{\Sigma_{k}}=\left.\varepsilon \cos \left(\vec{n}_{k}, x_{1}\right)\right|_{\Sigma_{k}}=\varepsilon \sin \left(\omega_{0} / 2-\theta_{k}\right)$, $k=1, \ldots, N-1$;

(3) from the representation $\partial G=\Gamma_{0}^{d} \cup \Gamma_{d}$ and by (2.1), we obtain $\left.\left(x_{i}-\varepsilon l_{i}\right) \cos \left(\vec{n}, x_{i}\right)\right|_{\Gamma_{0}^{d}}=-\varepsilon \sin \left(\omega_{0} / 2\right)$, so

$$
\begin{aligned}
& \int_{\partial G} a u^{2} r_{\varepsilon}^{\alpha-4}\left(x_{i}-\varepsilon l_{i}\right) \cos \left(\vec{n}, x_{i}\right) d s \\
& \quad=\int_{\Gamma_{d}} a u^{2} r_{\varepsilon}^{\alpha-4}\left(x_{i}-\varepsilon l_{i}\right) \cos \left(\vec{n}, x_{i}\right) d s-\varepsilon \sin \frac{\omega_{0}}{2} \int_{\Gamma_{0}^{d}} a u^{2} r_{\varepsilon}^{\alpha-4} d s .
\end{aligned}
$$

Hence and from (5.6) it follows that

$$
\begin{aligned}
& \frac{2-\alpha}{2} \int_{G} a r_{\varepsilon}^{\alpha-4}\left(x_{i}-\varepsilon l_{i}\right) \frac{\partial u^{2}}{\partial x_{i}} d x \\
= & \frac{(2-\alpha)(4-n-\alpha)}{2} \int_{G} a r_{\varepsilon}^{\alpha-4} u^{2} d x-\varepsilon \frac{2-\alpha}{2} \sin \left(\omega_{0} / 2\right) \int_{\Gamma_{0}^{d}} a u^{2} r_{\varepsilon}^{\alpha-4} d s \\
& +\frac{2-\alpha}{2} \int_{\Gamma_{d}} a u^{2} r_{\varepsilon}^{\alpha-4}\left(x_{i}-\varepsilon l_{i}\right) \cos \left(\vec{n}, x_{i}\right) d s \\
& +\varepsilon \sum_{k=1}^{N-1}[a]_{\Sigma_{k}} \int_{\Sigma_{k}} u^{2} r_{\varepsilon}^{\alpha-4} \sin \left(\omega_{0} / 2-\theta_{k}\right) d s .
\end{aligned}
$$

From (5.5), (5.7) we obtain

$$
\begin{array}{r}
\int_{G} a r_{\varepsilon}^{\alpha-2}|\nabla u|^{2} d x+\int_{\Sigma} r^{-1} r_{\varepsilon}^{\alpha-2} \beta(\phi) u^{2}(x) d s+\int_{\partial G} \alpha(x) r^{-1} r_{\varepsilon}^{\alpha-2} \gamma(\phi) u^{2}(x) d s \\
\quad+\varepsilon \frac{2-\alpha}{2} \sin \frac{\omega_{0}}{2} \int_{\Gamma_{0}^{d}} a u^{2} r_{\varepsilon}^{\alpha-4} d s=\frac{(2-\alpha)(4-n-\alpha)}{2} \int_{G} a r_{\varepsilon}^{\alpha-4} u^{2} d x
\end{array}
$$




$$
\begin{aligned}
& +\frac{2-\alpha}{2} \int_{\Gamma_{d}} a u^{2} r_{\varepsilon}^{\alpha-4}\left(x_{i}-\varepsilon l_{i}\right) \cos \left(\vec{n}, x_{i}\right) d s \\
& +\varepsilon \sum_{k=1}^{N-1}[a]_{\Sigma_{k}} \int_{\Sigma_{k}} u^{2} r_{\varepsilon}^{\alpha-4} \sin \left(\omega_{0} / 2-\theta_{k}\right) d s-\int_{G}(p u(x)+f(x)) r_{\varepsilon}^{\alpha-2} u(x) d x \\
& +\int_{\Sigma} r_{\varepsilon}^{\alpha-2} u(x) h(x) d s+\int_{\partial G} \alpha(x) r_{\varepsilon}^{\alpha-2} u(x) g(x) d s .
\end{aligned}
$$

Now we estimate the integral over $\Gamma_{d}$. Because on $\Gamma_{d}, r_{\varepsilon} \geq h r \geq h d$, so $(\alpha-3) \ln r_{\varepsilon} \leq(\alpha-3) \ln (h d)$, since $\alpha \leq 2$ we have $\left.r_{\varepsilon}^{\alpha-3}\right|_{\Gamma_{d}} \leq(h d)^{\alpha-3}$ and therefore

$$
\begin{aligned}
\frac{2-\alpha}{2} \int_{\Gamma_{d}} a u^{2} r_{\varepsilon}^{\alpha-4} & \left(x_{i}-\varepsilon l_{i}\right) \cos \left(\vec{n}, x_{i}\right) d s \\
& \leq \frac{2-\alpha}{2} \int_{\Gamma_{d}} a r_{\varepsilon}^{\alpha-3} u^{2} d s \leq \frac{2-\alpha}{2}(h d)^{\alpha-3} \int_{\Gamma_{d}} a u^{2} d s \\
& \leq c_{\delta} \int_{G_{d}} u^{2} d x+\delta \int_{G_{d}}|\nabla u|^{2} d x, \quad \forall \delta>0
\end{aligned}
$$

by $(2.10)$.

Further, by the Cauchy inequality and because of $\gamma(\phi) \geq \gamma_{0}>0$,

$$
\begin{aligned}
u g & =\left(r^{1 / 2} \frac{1}{\sqrt{\gamma(\phi)}}|g|\right)\left(r^{-1 / 2} \sqrt{\gamma(\phi)}|u|\right) \\
& \leq \frac{\delta_{1}}{2} r^{-1} \gamma(\phi) u^{2}+\frac{1}{2 \delta_{1} \gamma_{0}} r g^{2}(x), \quad \forall \delta_{1}>0 ;
\end{aligned}
$$

taking into account property (1) of $r_{\varepsilon}$ we obtain

$$
\begin{aligned}
\int_{\partial G} r_{\varepsilon}^{\alpha-2}|u||g| d s \leq & \frac{\delta_{1}}{2} \int_{\partial G} r_{\varepsilon}^{\alpha-2} \frac{1}{r} \gamma(\phi) u^{2} d s \\
& +\frac{1}{2 \delta_{1} \gamma_{0}} \int_{\partial G} r^{\alpha-1} g^{2}(x) d s, \quad \forall \delta_{1}>0 .
\end{aligned}
$$

Similarly, because of $\beta(\phi) \geq \beta_{0}>0$,

$$
\begin{aligned}
\int_{\Sigma} r_{\varepsilon}^{\alpha-2}|u||h(x)| d s \leq & \frac{\delta_{1}}{2} \int_{\Sigma} r_{\varepsilon}^{\alpha-2} \frac{1}{r} \beta(\phi) u^{2} d s \\
& +\frac{1}{2 \delta_{1} \beta_{0}} \int_{\Sigma_{0}} r^{\alpha-1} h^{2}(x) d s, \quad \forall \delta_{1}>0,
\end{aligned}
$$


and

$$
\begin{aligned}
\int_{G} r_{\varepsilon}^{\alpha-2} u f(x) d x \leq & \frac{\delta}{2} \int_{G} a r^{-2} r_{\varepsilon}^{\alpha-2} u^{2} d x \\
& +\frac{1}{2 a_{*} \delta} \int_{G} r^{\alpha} f^{2}(x) d x, \quad \forall \delta>0 .
\end{aligned}
$$

Now we use the representation $G=G_{0}^{d} \cup G_{d}$. Then

$$
\int_{G} p r_{\varepsilon}^{\alpha-2} u^{2}(x) d x=\int_{G_{0}^{d}} p r_{\varepsilon}^{\alpha-2} u^{2}(x) d x+\int_{G_{d}} p r_{\varepsilon}^{\alpha-2} u^{2}(x) d x .
$$

Therefore

$$
\begin{aligned}
\int_{G_{0}^{d}} p r_{\varepsilon}^{\alpha-2} u^{2}(x) d x & =\int_{G_{0}^{d}} a r^{-2} r_{\varepsilon}^{\alpha-2} u^{2}(x) \cdot \frac{p r^{2}}{a} d x \\
& \leq \frac{p^{*}}{a_{*}} d^{2} \int_{G_{0}^{d}} a r^{-2} r_{\varepsilon}^{\alpha-2} u^{2}(x) d x
\end{aligned}
$$

as well as, in view of property $(2)$ of $r_{\varepsilon}(x)$,

$$
\int_{G_{d}} p r_{\varepsilon}^{\alpha-2} u^{2}(x) d x \leq p^{*}(d / 2)^{\alpha-2} \int_{G} u^{2}(x) d x .
$$

Finally, we estimate the integral $\varepsilon \sum_{k=1}^{N-1}[a]_{\Sigma_{k}} \int_{\Sigma_{k}} u^{2} r_{\varepsilon}^{\alpha-4} \sin \left(\omega_{0} / 2-\theta_{k}\right) d s$. First, by (1.2), we get

$$
\begin{aligned}
\varepsilon \sum_{k=1}^{N-1}[a]_{\Sigma_{k}} \int_{\Sigma_{k}} u^{2} r_{\varepsilon}^{\alpha-4} \sin \left(\omega_{0} / 2-\theta_{k}\right) d s & \leq a_{0} \varepsilon \sum_{k=1}^{N-1} \int_{\Sigma_{k}} u^{2} r_{\varepsilon}^{\alpha-4} d s \\
& =a_{0} \varepsilon \int_{\Sigma} r_{\varepsilon}^{\alpha-4} u^{2}(x) d s .
\end{aligned}
$$

Further, we use the representation $\Sigma=\Sigma_{0}^{\varepsilon} \cup \Sigma_{\varepsilon}$. Then, by property (1) of $r_{\varepsilon}$ and in view of the inequality (3.27) of Corollary 3.4, we get

$$
\begin{aligned}
\varepsilon \int_{\Sigma_{0}^{\varepsilon}} r_{\varepsilon}^{\alpha-4} u^{2}(x) d s & \leq \int_{\Sigma_{0}^{\varepsilon}} r_{\varepsilon}^{\alpha-3} u^{2}(x) d s \leq C_{0}^{2} \operatorname{meas} \sigma \cdot \int_{0}^{\varepsilon} r^{\alpha+2 \varkappa+n-3} d r \\
& =\frac{C_{0}^{2} \operatorname{meas} \sigma}{\alpha+2 \varkappa+n-2} \varepsilon^{\alpha+2 \varkappa+n-2},
\end{aligned}
$$

because of $\alpha+2 \varkappa+n-2>0$, by our assumption (5.3). Similarly, for the integral over $\Sigma_{\varepsilon}$ we obtain 


$$
\begin{aligned}
& \varepsilon \int_{\Sigma_{\varepsilon}} r_{\varepsilon}^{\alpha-4} u^{2}(x) d s \leq \varepsilon \cdot \operatorname{meas} \sigma \cdot \int_{\varepsilon}^{R} r^{\alpha+2 \varkappa+n-4} d r \\
& =\operatorname{meas} \sigma \cdot \begin{cases}\frac{\varepsilon R^{\alpha+2 \varkappa+n-3}-\varepsilon^{\alpha+2 \varkappa+n-2}}{\alpha+2 \varkappa+n-3} & \text { if } \alpha+2 \varkappa+n-3 \neq 0, \\
\varepsilon \ln \frac{R}{\varepsilon} & \text { if } \alpha+2 \varkappa+n-3=0,\end{cases}
\end{aligned}
$$

where $R=\max _{1 \leq k \leq N-1} \operatorname{diam} \Sigma_{k}$. Thus, from (5.15)-(5.17) we get

$$
\varepsilon \cdot \sum_{k=1}^{N-1}[a]_{\Sigma_{k}} \int_{\Sigma_{k}} u^{2} r_{\varepsilon}^{\alpha-4} \sin \left(\omega_{0} / 2-\theta_{k}\right) d s \leq J(\varepsilon)
$$

where

$$
\times\left\{\begin{array}{rr}
\frac{R^{\alpha+2 \varkappa+n-3}}{\alpha+2 \varkappa+n-3}+\left(\frac{C_{0}^{2}}{\alpha+2 \varkappa+n-2}-\frac{1}{\alpha+2 \varkappa+n-3}\right) \varepsilon^{\alpha+2 \varkappa+n-3} \\
C_{0}^{2}+\ln \frac{R}{\varepsilon} & \text { if } \alpha+2 \varkappa+n-3 \neq 0,
\end{array}\right.
$$

provided that $\alpha+2 \varkappa+n-2>0$. Hence

Corollary 5.3.

$\lim _{\varepsilon \rightarrow+0} J(\varepsilon)=0, \quad$ so $\quad \lim _{\varepsilon \rightarrow+0} \varepsilon \cdot \sum_{k=1}^{N-1}[a]_{\Sigma_{k}} \int_{\Sigma_{k}} u^{2} r_{\varepsilon}^{\alpha-4} \sin \left(\omega_{0} / 2-\theta_{k}\right) d s=0$.

As a result, from (5.8)-(5.18) we obtain

$$
\begin{aligned}
& \int_{G} a r_{\varepsilon}^{\alpha-2}|\nabla u|^{2} d x+\int_{\Sigma} r^{-1} r_{\varepsilon}^{\alpha-2} \beta(\phi) u^{2}(x) d s \\
+ & \int_{\partial G} \alpha(x) r^{-1} r_{\varepsilon}^{\alpha-2} \gamma(\phi) u^{2}(x) d s+\varepsilon \frac{2-\alpha}{2} \sin \frac{\omega_{0}}{2} \int_{\Gamma_{0}^{d}} a u^{2} r_{\varepsilon}^{\alpha-4} d s \\
\leq & J(\varepsilon)+\frac{(2-\alpha)(4-n-\alpha)}{2} \int_{G} a r_{\varepsilon}^{\alpha-4} u^{2} d x \\
+ & \left(\frac{\delta}{2}+\frac{p^{*}}{a_{*}} d^{2}\right) \int_{G_{0}^{d}} a r^{-2} r_{\varepsilon}^{\alpha-2} u^{2} d x+c\left(p^{*}, a_{*}, \alpha, d\right) \int_{G}\left(a|\nabla u|^{2}+u^{2}\right) d x \\
+ & \frac{\delta_{1}}{2}\left\{\int_{\Sigma} r^{-1} r_{\varepsilon}^{\alpha-2} \beta(\phi) u^{2}(x) d s+\int_{\partial G} \alpha(x) r^{-1} r_{\varepsilon}^{\alpha-2} \gamma(\phi) u^{2}(x) d s\right\}
\end{aligned}
$$




$$
\begin{aligned}
& +\frac{1}{2 \delta_{1} \gamma_{0}} \int_{\partial G} r^{\alpha-1} g^{2}(x) d s+\frac{1}{2 \delta_{1} \beta_{0}} \int_{\Sigma} r^{\alpha-1} h^{2}(x) d s \\
& +\frac{1}{2 a_{*} \delta} \int_{G} r^{\alpha} f^{2}(x) d x, \quad \forall \delta, \delta_{1}>0 .
\end{aligned}
$$

Now it is clear that it is sufficient to consider the case $\alpha<4-n$. First we apply Lemma 2.6. Then from (5.20) we get

$$
\begin{aligned}
& \left(1-\frac{2(2-\alpha)(4-\alpha-n)}{(4-n-\alpha)^{2}+4 \lambda(\lambda+n-2)}\right)\left\{\int_{G} a r_{\varepsilon}^{\alpha-2}|\nabla u|^{2} d x\right. \\
& \left.+\int_{\Sigma} \frac{1}{r} r_{\varepsilon}^{\alpha-2} \beta(\phi) u^{2}(x) d s+\int_{\partial G} \alpha(x) \frac{1}{r} r_{\varepsilon}^{\alpha-2} \gamma(\phi) u^{2}(x) d s\right\} \\
& \leq J(\varepsilon)+\left(\frac{\delta}{2}+\frac{p^{*}}{a_{*}} d^{2}\right) \int_{G_{0}^{d}} a r^{-2} r_{\varepsilon}^{\alpha-2} u^{2} d x \\
& +c\left(p^{*}, a_{*}, \alpha, d\right) \int_{G}\left(a|\nabla u|^{2}+u^{2}\right) d x+\frac{1}{2 \delta_{1} \gamma_{0}} \int_{\partial G} r^{\alpha-1} g^{2}(x) d s \\
& +\frac{\delta_{1}}{2}\left\{\int_{\Sigma} r^{-1} r_{\varepsilon}^{\alpha-2} \beta(\phi) u^{2}(x) d s+\int_{\partial G} \alpha(x) r^{-1} r_{\varepsilon}^{\alpha-2} \gamma(\phi) u^{2}(x) d s\right\} \\
& +\frac{1}{2 \delta_{1} \beta_{0}} \int_{\Sigma} r^{\alpha-1} h^{2}(x) d s+\frac{1}{2 a_{*} \delta} \int_{G} r^{\alpha} f^{2}(x) d x, \quad \forall \delta, \delta_{1}>0, \forall \varepsilon>0 .
\end{aligned}
$$

But we have

$$
\frac{2(2-\alpha)(4-\alpha-n)}{(4-n-\alpha)^{2}+4 \lambda(\lambda+n-2)}<1
$$

provided $\alpha>4-n-2 \lambda$; this is indeed satisfied, in view of $\alpha>2-n-2 \varkappa$ and $0<\varkappa \leq \lambda-1$. Therefore (5.22) is true. Now we apply Lemma 2.7 and choose $\delta_{1}=\frac{2}{\lambda(\lambda+n-2)}\left(\frac{\delta}{2}+\frac{p^{*}}{a_{*}} d^{2}\right)$. As a result we obtain

$$
\left(1-\frac{2(2-\alpha)(4-\alpha-n)}{(4-n-\alpha)^{2}+4 \lambda(\lambda+n-2)}\right)\left\{\int_{G} a r_{\varepsilon}^{\alpha-2}|\nabla u|^{2} d x\right.
$$

$$
\left.+\int_{\Sigma} \frac{1}{r} r_{\varepsilon}^{\alpha-2} \beta(\phi) u^{2}(x) d s+\int_{\partial G} \alpha(x) \frac{1}{r} r_{\varepsilon}^{\alpha-2} \gamma(\phi) u^{2}(x) d s\right\}
$$

$$
\begin{aligned}
\leq & J(\varepsilon)+c\left(p^{*}, a_{*}, \alpha, d\right) \int_{G}\left(a|\nabla u|^{2}+u^{2}\right) d x \\
& +\frac{1}{2 \delta_{1} \gamma_{0}} \int_{\partial G} r^{\alpha-1} g^{2}(x) d s+\frac{2}{\lambda(\lambda+n-2)}\left(\frac{\delta}{2}+\frac{p^{*}}{a_{*}} d^{2}\right)\left\{\int_{G} a r_{\varepsilon}^{\alpha-2}|\nabla u|^{2} d x\right.
\end{aligned}
$$




$$
\begin{aligned}
& \left.+\int_{\Sigma} r^{-1} r_{\varepsilon}^{\alpha-2} \beta(\phi) u^{2}(x) d s+\int_{\partial G} \alpha(x) r^{-1} r_{\varepsilon}^{\alpha-2} \gamma(\phi) u^{2}(x) d s\right\} \\
& +\frac{1}{2 \delta_{1} \beta_{0}} \int_{\Sigma} r^{\alpha-1} h^{2}(x) d s+\frac{1}{2 a_{*} \delta} \int_{G} r^{\alpha} f^{2}(x) d x, \quad \forall \delta, \varepsilon>0 .
\end{aligned}
$$

Now we can choose

$$
d=\sqrt{\frac{\delta a_{*}}{2 p^{*}}}, \quad \delta=\frac{\lambda(\lambda+n-2)}{4}\left(1-\frac{2(2-\alpha)(4-\alpha-n)}{(4-n-\alpha)^{2}+4 \lambda(\lambda+n-2)}\right) .
$$

Thus we get

$$
\begin{gathered}
\int_{G} a r_{\varepsilon}^{\alpha-2}|\nabla u|^{2} d x+\int_{\Sigma} \frac{1}{r} r_{\varepsilon}^{\alpha-2} \beta(\phi) u^{2}(x) d s+\int_{\partial G} \alpha(x) \frac{1}{r} r_{\varepsilon}^{\alpha-2} \gamma(\phi) u^{2}(x) d s \\
\leq C\left(p^{*}, n, a_{*}, \alpha, \lambda\right)\left\{J(\varepsilon)+\int_{G}\left(a|\nabla u|^{2}+u^{2}+r^{\alpha} f^{2}(x)\right) d x\right. \\
\left.+\frac{1}{\gamma_{0}} \int_{\partial G} \alpha(x) r^{\alpha-1} g^{2}(x) d s+\frac{1}{\beta_{0}} \int_{\Sigma} r^{\alpha-1} h^{2}(x) d s\right\}, \quad \forall \varepsilon>0 .
\end{gathered}
$$

Now we can let $\varepsilon \rightarrow+0$ by the Fatou theorem. From Corollary 5.3 it follows that

$$
\begin{aligned}
\int_{G} a r^{\alpha-2}|\nabla u|^{2} d x & +\int_{\Sigma} r^{\alpha-3} \beta(\phi) u^{2}(x) d s+\int_{\partial G} \alpha(x) r^{\alpha-3} \gamma(\phi) u^{2}(x) d s \\
\leq & C\left(p^{*}, n, a_{*}, \alpha, \lambda\right)\left\{\int_{G}\left(a|\nabla u|^{2}+u^{2}+r^{\alpha} f^{2}(x)\right) d x\right. \\
& \left.+\frac{1}{\gamma_{0}} \int_{\partial G} \alpha(x) r^{\alpha-1} g^{2}(x) d s+\frac{1}{\beta_{0}} \int_{\Sigma} r^{\alpha-1} h^{2}(x) d s\right\} .
\end{aligned}
$$

Now applying Theorem 5.1, by the Hardy-Friedrichs-Wirtinger inequality (2.5), from (5.25) we get the desired estimate (5.4).

\section{Local integral weighted estimates}

THEOREM 6.1. Let $u$ be a weak solution of problem $(L)$ and $\lambda>1$ be as in (1.3). Let assumptions (a)-(c) be satisfied and $\mathcal{D}=\emptyset$. Then $u \in \stackrel{\circ}{\mathbf{W}}_{2-n}^{1}(G)$ and there are $d \in(0,1)$, a constant $C>0$ depending only on $n, s, \lambda, a_{*}, G, \Sigma$ and $a c=\operatorname{const}\left(n, \lambda, a_{*}, p^{*}\right)$ such that for all $\varrho \in(0, d)$,

$$
\begin{gathered}
\int_{G_{0}^{\varrho}}\left(a r^{2-n}|\nabla u|^{2}+r^{-n} u^{2}(x)\right) d x+\int_{\Sigma_{0}^{\varrho}} r^{1-n} \beta(\phi) u^{2}(x) d s \\
+\int_{\Gamma_{0}^{\varrho}} r^{1-n} \gamma(\phi) u^{2}(x) d s
\end{gathered}
$$




$$
\leq C\left(\|u\|_{2, G}^{2}+f_{0}^{2}+\frac{1}{\gamma_{0}} g_{0}^{2}+\frac{1}{\beta_{0}} h_{0}^{2}\right) \cdot \begin{cases}\varrho^{2 \lambda} & \text { if } s>\lambda, \\ \varrho^{2 \lambda} \ln ^{c}(1 / \varrho) & \text { if } s=\lambda \\ \varrho^{2 s} & \text { if } s<\lambda .\end{cases}
$$

Proof. From Theorem 5.2 it follows that $u \in \stackrel{\circ}{\mathbf{W}}_{2-n}^{1}(G)$, so it is enough to prove the estimate (6.1). Setting $\eta(x)=r^{2-n} u(x)$ in (II) ${ }_{\text {loc }}$, by the definition (2.6) we obtain

$$
\begin{aligned}
U(\varrho)= & \left.\varrho \int_{\Omega} a u(x) \frac{\partial u}{\partial r}\right|_{r=\varrho} d \Omega+\int_{\Gamma_{0}^{\varrho}} \alpha(x) r^{2-n} u(x) g(x) d s \\
& +\int_{\Sigma_{0}^{\varrho}} r^{2-n} u(x) h(x) d s \\
& +\int_{G_{0}^{\varrho}}\left\{(n-2) a r^{-n} u(x) x_{i} u_{x_{i}}-p r^{2-n} u^{2}(x)-r^{2-n} u(x) f(x)\right\} d x .
\end{aligned}
$$

By the Gauss-Ostrogradskil divergence theorem, we find that

$$
\begin{aligned}
& \text { (6.3) } \quad(n-2) \int_{G_{0}^{\varrho}} a r^{-n} u(x) x_{i} u_{x_{i}} d x=\frac{n-2}{2} \int_{G_{0}^{\varrho}} a r^{-n} x_{i} \frac{\partial u^{2}}{\partial x_{i}} d x \\
& =\frac{n-2}{2}\left\{-\int_{G_{0}^{\varrho}} a u^{2}(x)\left(n r^{-n}-n r^{-n}\right) d x+\varrho^{-n} \int_{\Omega_{\varrho}} a u^{2}(x) x_{i} \cos \left(r, x_{i}\right) d \Omega_{\varrho}\right.
\end{aligned}
$$$$
\left.+\sum_{k=1}^{N-1}[a]_{\Sigma_{k}} \int_{\left(\Sigma_{k}\right)_{0}^{\varrho}} r^{-n} u^{2}(x) x_{i} \cos \left(n_{k}, x_{i}\right) d s+\int_{\Gamma_{0}^{\varrho}} \alpha(x) a r^{-n} u^{2}(x) x_{i} \cos \left(n, x_{i}\right) d s\right\} .
$$

By Lemma 2.1,

$$
\begin{aligned}
& \left.x_{i} \cos \left(n_{k}, x_{i}\right)\right|_{\Sigma_{k}}=0, \quad k=1, \ldots, N-1 ; \\
& \left.x_{i} \cos \left(n, x_{i}\right)\right|_{\Gamma_{0}^{\varrho}}=0,\left.\quad x_{i} \cos \left(r, x_{i}\right)\right|_{\Omega_{\varrho}}=\varrho,
\end{aligned}
$$

we have

$$
(n-2) \int_{G_{0}^{\varrho}} a r^{-n} u(x) x_{i} u_{x_{i}} d x=\frac{n-2}{2} \int_{\Omega} a u^{2}(x) d \Omega .
$$

Because of Lemma 2.5, from (6.2)-(6.4) it follows that

$$
\begin{aligned}
U(\varrho) \leq & \frac{\varrho}{2 \lambda} U^{\prime}(\varrho)+\int_{\Gamma_{0}^{\varrho}} r^{2-n}|u(x)| \cdot|g(x)| d s+\int_{\Sigma_{0}^{\varrho}} r^{2-n}|u(x)| \cdot|h(x)| d s \\
& +p^{*} \int_{G_{0}^{\varrho}} r^{2-n} u^{2} d x+\int_{G_{0}^{\varrho}} r^{2-n}|u(x)| \cdot|f(x)| d x .
\end{aligned}
$$

We shall obtain an upper bound for each integral on the right. First, applying the Cauchy and Hardy-Friedrichs-Wirtinger inequality (2.5), we have, for any $\delta>0$, 
(6.6)

$$
\begin{aligned}
\int_{\Gamma_{0}^{O}} r^{2-n}|u||g| d s & =\int_{\Gamma_{0}^{o}}\left(r^{(1-n) / 2} \sqrt{\gamma(\phi)}|u|\right)\left(r^{(3-n) / 2} \frac{1}{\sqrt{\gamma(\phi)}}|g|\right) d s \\
& \leq \frac{\delta}{2} \int_{\Gamma_{0}^{O}} r^{1-n} \gamma(\phi)|u|^{2} d s+\frac{1}{2 \delta \gamma_{0}} \int_{\Gamma_{0}^{O}} r^{3-n}|g|^{2} d s
\end{aligned}
$$

$$
\begin{aligned}
\int_{\Sigma_{0}^{\varrho}} r^{2-n}|u||h| d s & =\int_{\Sigma_{0}^{\varrho}}\left(r^{(1-n) / 2} \sqrt{\beta(\phi)}|u|\right)\left(r^{(3-n) / 2} \frac{1}{\sqrt{\beta(\phi)}}|g|\right) d s \\
& \leq \frac{\delta}{2} \int_{\Sigma_{0}^{\varrho}} r^{1-n} \beta(\phi)|u|^{2} d s+\frac{1}{2 \delta \beta_{0}} \int_{\Sigma_{0}^{\varrho}} r^{3-n}|h|^{2} d s
\end{aligned}
$$

$$
\begin{aligned}
\int_{G_{0}^{\varrho}} r^{2-n}|u(x)||f(x)| d x & \leq \frac{\delta}{2 a_{*}} \int_{G_{0}^{\varrho}} a r^{-n}|u|^{2} d x+\frac{1}{2 \delta} \int_{G_{0}^{\varrho}} r^{4-n}|f|^{2} d x \\
& \leq \frac{\delta}{2 a_{*} \lambda(\lambda+n-2)} U(\varrho)+\frac{1}{2 \delta} \int_{G_{0}^{\varrho}} r^{4-n}|f|^{2} d x \\
p^{*} \int_{G_{0}^{\varrho}} r^{2-n} u^{2} d x & \leq p^{*} \varrho^{2} \int_{G_{0}^{\varrho}} r^{-n} u^{2} d x \leq \frac{p^{*}}{a_{*} \lambda(\lambda+n-2)} \varrho^{2} U(\varrho) .
\end{aligned}
$$

Thus from (6.5)-(6.9) we get

$$
+\frac{1}{2 \delta}\left\{\int_{G_{0}^{\varrho}} r^{4-n}|f|^{2} d x+\frac{1}{\gamma_{0}} \int_{\Gamma_{0}^{\varrho}} r^{3-n}|g|^{2} d s+\frac{1}{\beta_{0}} \int_{\Sigma_{0}^{\varrho}} r^{3-n}|h|^{2} d s\right\}, \quad \forall \delta>0 .
$$

But, by condition (c),

$$
\begin{aligned}
\int_{G_{0}^{\varrho}} r^{4-n}|f|^{2} d x+\frac{1}{\gamma_{0}} \int_{\Gamma_{0}^{\varrho}} r^{3-n}|g|^{2} d s & +\frac{1}{\beta_{0}} \int_{\Sigma_{0}^{\varrho}} r^{3-n}|h|^{2} d s \\
& \leq \frac{c_{0}(G)}{2 s}\left(f_{0}^{2}+\frac{1}{\gamma_{0}} g_{0}^{2}+\frac{1}{\beta_{0}} h_{0}^{2}\right) \cdot \varrho^{2 s} .
\end{aligned}
$$

Thus from (6.10) we have the differential inequality (CP) of $\S 2.4$ with

$$
\mathcal{P}(\varrho)=\frac{2 \lambda}{\varrho} \cdot\left\{1-c_{1}\left(n, \lambda, a_{*}, p^{*}\right)\left(\delta+\varrho^{2}\right)\right\}, \forall \delta>0 ; \quad \mathcal{N}(\varrho) \equiv 0 ;
$$

$$
U_{0}=C\left\{\int_{G}\left(u^{2}+\left(1+r^{4-n}\right) f^{2}(x)\right) d x+\int_{\Sigma} r^{3-n} h^{2}(x) d s+\int_{\partial G} r^{3-n} g^{2}(x) d s\right\},
$$

by (5.4) with $\alpha=4-n$. 
1) $s>\lambda$. Choosing $\delta=\varrho^{\varepsilon}, \forall \varepsilon>0$, we get

$$
\begin{aligned}
& \mathcal{P}(\varrho)=\frac{2 \lambda}{\varrho}-2 \lambda c_{1}\left(n, \lambda, a_{*}, p^{*}\right)\left(\varrho^{\varepsilon-1}+\varrho\right) ; \\
& \mathcal{Q}(\varrho)=\frac{c_{0} \lambda}{2 s}\left(f_{1}^{2}+\frac{1}{\gamma_{0}} g_{1}^{2}+\frac{1}{\beta_{0}} h_{1}^{2}\right) \cdot \varrho^{2 s-1-\varepsilon} .
\end{aligned}
$$

Now for $0<\varrho<\tau<d$ we have

$$
\begin{aligned}
-\int_{\varrho}^{\tau} \mathcal{P}(s) d s & =-2 \lambda \ln \left(\frac{\tau}{\varrho}\right)+\left.2 \lambda c_{1}\left(\frac{s^{\varepsilon}}{\varepsilon}+\frac{s^{2}}{2}\right)\right|_{\varrho} ^{\tau} \\
& \leq \ln \left(\frac{\varrho}{\tau}\right)^{2 \lambda}+2 \lambda c_{1}\left(\frac{d^{\varepsilon}}{\varepsilon}+\frac{d^{2}}{2}\right),
\end{aligned}
$$

so

$$
\begin{gathered}
\exp \left(-\int_{\varrho}^{d} \mathcal{P}(\tau) d \tau\right) \leq\left(\frac{\varrho}{d}\right)^{2 \lambda} \exp \left\{2 \lambda c_{1}\left(\frac{d^{\varepsilon}}{\varepsilon}+\frac{d^{2}}{2}\right)\right\}=K_{0}\left(\frac{\varrho}{d}\right)^{2 \lambda} ; \\
\exp \left(-\int_{\varrho}^{\tau} \mathcal{P}(\tau) d \tau\right) \leq K_{0}\left(\frac{\varrho}{\tau}\right)^{2 \lambda},
\end{gathered}
$$

where $K_{0}=\exp \left\{2 \lambda c_{1}\left(d^{\varepsilon} / \varepsilon+d^{2} / 2\right)\right\}$. We have as well

$$
\begin{aligned}
\int_{\varrho}^{d} \mathcal{Q}(\tau) \exp \left(-\int_{\varrho}^{\tau} \mathcal{P}(\sigma) d \sigma\right) d \tau & \\
& \leq \frac{\lambda c_{0} K_{0}}{2 s}\left(f_{0}^{2}+\frac{1}{\gamma_{0}} g_{0}^{2}+\frac{1}{\beta_{0}} h_{0}^{2}\right) \varrho^{2 \lambda} \int_{\varrho}^{d} \tau^{2 s-2 \lambda-\varepsilon-1} d \tau \\
& \leq \frac{\lambda c_{0} K_{0}}{2 s}\left(f_{0}^{2}+\frac{1}{\gamma_{0}} g_{0}^{2}+\frac{1}{\beta_{0}} h_{0}^{2}\right) \cdot \frac{d^{s-\lambda}}{s-\lambda} \varrho^{2 \lambda},
\end{aligned}
$$

since $s>\lambda$ and we can choose $\varepsilon=s-\lambda$. Therefore in our case $K_{0}=$ $\exp \left\{2 \lambda c_{1}\left(\frac{d^{s-\lambda}}{s-\lambda}+\frac{d^{2}}{2}\right)\right\}$.

Now we apply Theorem 2.8. Then from (2.13), by the above inequalities and (2.5) for $\alpha=4-n$, we obtain the statement of (6.1) for $s>\lambda$.

2) $s=\lambda$. Taking in (6.11) any function $\delta(\varrho)>0$ instead of $\delta>0$, we obtain the problem $(\mathrm{CP})$ with

$$
\begin{aligned}
& \mathcal{P}(\varrho)=\frac{2 \lambda\left(1-c_{1} \delta(\varrho)\right)}{\varrho}-2 \lambda c_{1} \varrho ; \quad \mathcal{N}(\varrho)=0 ; \\
& \mathcal{Q}(\varrho)=\frac{c_{0}}{2}\left(f_{0}^{2}+\frac{1}{\gamma_{0}} g_{0}^{2}+\frac{1}{\beta_{0}} h_{0}^{2}\right) \cdot \delta^{-1}(\varrho) \varrho^{2 \lambda-1} .
\end{aligned}
$$

We choose $\delta(\varrho)=\frac{1}{2 \lambda \ln (e d / \varrho)}, 0<\varrho<d$, where $e$ is the Euler number. Then 
we obtain

$$
\begin{aligned}
-\int_{\varrho}^{d} \mathcal{P}(\tau) d \tau & \leq \ln \left(\frac{\varrho}{d}\right)^{2 \lambda}+c_{1} \int_{\varrho}^{d} \frac{d \tau}{\tau \ln (e d / \tau)}+\lambda c_{1} d^{2} \\
& =\ln \left(\frac{\varrho}{d}\right)^{2 \lambda}+c_{1} \ln \ln \left(\frac{e d}{\varrho}\right)+\lambda c_{1} d^{2}
\end{aligned}
$$

SO

$$
\exp \left(-\int_{\varrho}^{d} \mathcal{P}(\tau) d \tau\right) \leq\left(\frac{\varrho}{d}\right)^{2 \lambda} \ln ^{c_{1}}\left(\frac{e d}{\varrho}\right) \exp \left(\lambda c_{1} d^{2}\right)
$$

In this case we also have

$$
\begin{aligned}
& \int_{\varrho}^{d} \mathcal{Q}(\tau) \exp \left(-\int_{\varrho}^{\tau} \mathcal{P}(\sigma) d \sigma\right) d \tau \\
& \quad \leq \lambda c_{0}\left(f_{0}^{2}+\frac{1}{\gamma_{0}} g_{0}^{2}+\frac{1}{\beta_{0}} h_{0}^{2}\right) e^{\lambda c_{1} d^{2}} \cdot \varrho^{2 \lambda} \ln ^{1+c_{1}}\left(\frac{e d}{\varrho}\right) \int_{\varrho}^{d} \frac{d \tau}{\tau} \\
& \quad \leq \lambda c_{0}\left(f_{0}^{2}+\frac{1}{\gamma_{0}} g_{0}^{2}+\frac{1}{\beta_{0}} h_{0}^{2}\right) e^{\lambda c_{1} d^{2}} \cdot \varrho^{2 \lambda} \ln ^{2+c_{1}}\left(\frac{e d}{\varrho}\right) .
\end{aligned}
$$

Now we apply Theorem 2.8, and from (2.13), by the inequalities obtained, we get

$$
U(\varrho) \leq c_{2}\left(U_{0}+f_{0}^{2}+\frac{1}{\gamma_{0}} g_{0}^{2}+\frac{1}{\beta_{0}} h_{0}^{2}\right) \varrho^{2 \lambda} \ln ^{c} \frac{1}{\varrho}, \quad 0<\varrho<d<\frac{1}{e},
$$

where $c=\operatorname{const}\left(n, \lambda, a_{*}, p^{*}\right)$. Thus we proved the statement of (6.1) for $s=\lambda$.

3) $0<s<\lambda$. Now similar to case 1 ), by (6.11) we have

$$
\exp \left(-\int_{\varrho}^{d} \mathcal{P}(\tau) d \tau\right) \leq\left(\frac{\varrho}{d}\right)^{2 \lambda\left(1-c_{1} \delta\right)} \cdot e^{\lambda d^{2}}
$$

In this case we also have

$$
\begin{aligned}
& \int_{\varrho}^{d} \mathcal{Q}(\tau) \exp \left(-\int_{\varrho}^{\tau} \mathcal{P}(\sigma) d \sigma\right) d \tau \\
& \quad \leq \frac{\lambda c_{0} e^{\lambda d^{2}}}{2 s \delta}\left(f_{0}^{2}+\frac{1}{\gamma_{0}} g_{0}^{2}+\frac{1}{\beta_{0}} h_{0}^{2}\right) \cdot \varrho^{2 \lambda\left(1-c_{1} \delta\right)} \int_{\varrho}^{d} \tau^{2 s-2 \lambda\left(1-c_{1} \delta\right)-1} d \tau \\
& \quad \leq c_{0} c_{3}\left(n, \lambda, a_{*}, p^{*}, d, s\right)\left(f_{0}^{2}+\frac{1}{\gamma_{0}} g_{0}^{2}+\frac{1}{\beta_{0}} h_{0}^{2}\right) \cdot \varrho^{2 s}
\end{aligned}
$$

if we choose $\delta \in\left(0, \frac{\lambda-s}{\lambda c_{1}}\right)$. 
Now we apply Theorem 2.8, and then from (2.13), by the above inequalities, we obtain

$$
\begin{aligned}
U(\varrho) & \leq c_{4}\left(U_{0} \varrho^{2 \lambda\left(1-c_{1} \delta\right)}+\left(f_{0}^{2}+\frac{1}{\gamma_{0}} g_{0}^{2}+\frac{1}{\beta_{0}} h_{0}^{2}\right) \cdot \varrho^{2 s}\right) \\
& \leq c_{5}\left(U_{0}+f_{0}^{2}+\frac{1}{\gamma_{0}} g_{0}^{2}+\frac{1}{\beta_{0}} h_{0}^{2}\right) \varrho^{2 s} .
\end{aligned}
$$

Thus we have proved the statement of (6.1) for $s<\lambda$.

7. The power modulus of continuity at the conical point for weak solutions

Proof of Theorem 1.2. Define

$$
\psi(\varrho)= \begin{cases}\varrho^{\lambda} & \text { if } s>\lambda, \\ \varrho^{\lambda} \ln ^{c}(1 / \varrho) & \text { if } s=\lambda, \\ \varrho^{s} & \text { if } s<\lambda,\end{cases}
$$

for $0<\varrho<d$, where $c>0$ is determined by Theorem 6.1..

By Theorem 4.1 we have

$$
\begin{aligned}
\sup _{G_{0}^{\varrho / 2}}|u(x)| \leq C\left\{\varrho^{-n / 2}\|u\|_{2, G_{0}^{\varrho}}+\varrho^{2(1-n / q)}\|f\|_{q / 2, G_{0}^{\varrho}}\right. & \\
& +\varrho\left(\|g\|_{\infty, \Gamma_{0}^{\varrho}}+\|h\|_{\left.\left.\infty, \Sigma_{0}^{\varrho}\right)\right\}}\right.
\end{aligned}
$$

where $C=C\left(n, a_{*}, a^{*}, q, G\right)$ and $q>n$. Now by Theorem 6.1,

$$
\begin{aligned}
& \text { 7.3) } \quad \varrho^{-n / 2}\|u\|_{2, G_{0}^{\varrho}} \leq 2^{n / 2}\left(\int_{G_{0}^{\varrho}} r^{-n} u^{2}(x) d x\right)^{1 / 2} \\
& \leq C\left(\|u\|_{2, G}+\|f\|_{2, G}+\|g\|_{2, \partial G}+\|h\|_{2, \Sigma_{0}}+f_{0}+\frac{1}{\sqrt{\gamma_{0}}} g_{0}+\frac{1}{\sqrt{\beta_{0}}} h_{0}\right) \psi(\varrho) .
\end{aligned}
$$

Further, by assumption (c),

$$
\begin{aligned}
\varrho^{2(1-n / q)}\|f\|_{q / 2, G_{0}^{o}}+\varrho\left(\|g\|_{\infty, \Gamma_{0}^{\varrho}}\right. & \left.+\|h\|_{\infty, \Sigma_{0}^{\varrho}}\right) \\
& \leq c\left(f_{0}+\frac{1}{\sqrt{\gamma_{0}}} g_{0}+\frac{1}{\sqrt{\beta_{0}}} h_{0}\right) \psi(\varrho) .
\end{aligned}
$$

From (7.2)-(7.4) it follows that

$$
\begin{aligned}
\sup _{G_{\varrho / 4}^{\varrho / 2}}|u(x)| \leq C\left(\|u\|_{2, G}+\|f\|_{2, G}+\right. & \|g\|_{2, \partial G}+\|h\|_{2, \Sigma_{0}} \\
& \left.+f_{0}+\frac{1}{\sqrt{\gamma_{0}}} g_{0}+\frac{1}{\sqrt{\beta_{0}}} h_{0}\right) \psi(\varrho) .
\end{aligned}
$$

Putting now $|x|=\frac{1}{3} \varrho$ we finally obtain the desired estimate (1.4). 
Now we consider the sets $G_{\varrho / 4}^{2 \varrho}$ and $G_{\varrho / 2}^{\varrho} \subset G_{\varrho / 4}^{2 \varrho}, \varrho>0$. We perform the change of variables $x=\varrho x^{\prime}$ and $u\left(\varrho x^{\prime}\right)=\psi(\varrho) v\left(x^{\prime}\right)$. Then the function $v\left(x^{\prime}\right)$ satisfies

$$
\left(L^{\prime \prime}\right) \begin{cases}a_{i} \Delta^{\prime} v_{i}+p_{i} \varrho^{2} v_{i}\left(x^{\prime}\right)=\frac{\varrho^{2}}{\psi(\varrho)} f_{i}\left(\varrho x^{\prime}\right), & \quad x^{\prime} \in\left(G_{i}\right)_{1 / 2}^{1}, i=1, \ldots, N \\ {\left[v\left(x^{\prime}\right)\right]_{\left(\Sigma_{k}\right)_{1 / 2}^{1}=0,}} & k=1, \ldots, N-1 ; \\ {\left[a \frac{\partial v}{\partial n_{k}^{\prime}}\right]_{\left(\Sigma_{k}\right)_{1 / 2}^{1}}+\frac{1}{\left|x^{\prime}\right|} \beta_{k}(\phi) v\left(x^{\prime}\right)=\frac{\varrho}{\psi(\varrho)} h_{k}\left(\varrho x^{\prime}\right),} & \quad x^{\prime} \in\left(\Sigma_{k}\right)_{1 / 2}^{1}, k=1, \ldots, N-1 ; \\ \alpha\left(x^{\prime}\right) \cdot a \frac{\partial v}{\partial n^{\prime}}+\frac{1}{\left|x^{\prime}\right|} \gamma(\phi) v\left(x^{\prime}\right)=\frac{\varrho}{\psi(\varrho)} g\left(\varrho x^{\prime}\right), \quad x^{\prime} \in \Gamma_{1 / 2}^{1} .\end{cases}
$$

By the Sobolev imbedding theorems,

$$
\sup _{x^{\prime} \in G_{1 / 2}^{1}}\left|\nabla^{\prime} v\left(x^{\prime}\right)\right| \leq c\|v\|_{\mathbf{W}^{2, q}\left(G_{1 / 2}^{1}\right)}, \quad q>n .
$$

By the local $L^{p}$ a priori estimate [13], for the solution of the equation of $\left(L^{\prime \prime}\right)$ in the domains $\left(G_{i}\right)_{1 / 4}^{2}, i=1, \ldots, N$, and near smooth portions of the boundaries $\left(\Sigma_{k}\right)_{1 / 4}^{2}, k=1, \ldots, N-1$, and $\Gamma_{1 / 4}^{2}$ we have

$$
\begin{aligned}
\|v\|_{\mathbf{W}^{2, q}\left(G_{1 / 2}^{1}\right)} \leq & c\left\{\frac{\varrho^{2}}{\psi(\varrho)}\|f\|_{\mathbf{L}^{q}\left(G_{1 / 4}^{2}\right)}+\frac{\varrho}{\psi(\varrho)}\|h\|_{\mathbf{W}^{1-1 / q, q}\left(\Sigma_{1 / 4}^{2}\right)}\right. \\
& \left.+\frac{\varrho}{\psi(\varrho)}\|g\|_{\mathbf{W}^{1-1 / q, q}\left(\Gamma_{1 / 4}^{2}\right)}+\|v\|_{\mathbf{L}^{q}\left(G_{1 / 4}^{2}\right)}\right\} .
\end{aligned}
$$

Returning back to the variables $x$, from (7.5) and (7.6), it follows that

$$
\begin{aligned}
\sup _{G_{\varrho / 2}^{\varrho}}|\nabla u| \leq & c \varrho^{-1}\left\{\varrho^{-n / q}\|u\|_{\mathbf{L}^{q}\left(G_{\varrho / 4}^{2 \varrho}\right)}+\varrho^{2-n / q}\|f\|_{q, G_{\varrho / 4}^{2 \varrho}}\right. \\
& \left.+\varrho^{2-n / q}\|g\|_{\mathbf{V}_{q, 0}^{1-1 / q}\left(\Gamma_{\varrho / 4}^{2 \varrho}\right)}+\varrho^{2-n / p}\|h\|_{\mathbf{V}_{q, 0}^{1-1 / q}\left(\Sigma_{\varrho / 4}^{2 \varrho}\right)}\right\}
\end{aligned}
$$

and

$$
\begin{aligned}
\varrho^{2-n / q}\|u\|_{\mathbf{V}_{q, 0}^{2}\left(G_{\varrho / 2}^{\varrho}\right)} \leq & c\left\{\varrho^{-n / q}\|u\|_{\mathbf{L}^{q}\left(G_{\varrho / 4}^{2 \varrho}\right)}+\varrho^{2-n / q}\|f\|_{q, G_{\varrho / 4}^{2 \varrho}}\right. \\
& \left.+\varrho^{2-n / q}\|g\|_{\mathbf{V}_{q, 0}^{1-1 / q}\left(\Gamma_{\varrho / 4}^{2 \varrho}\right)}+\varrho^{2-n / q}\|h\|_{\mathbf{V}_{q, 0}^{1-1 / q}\left(\Sigma_{\varrho / 4}^{2 \varrho}\right)}\right\}
\end{aligned}
$$

or

$$
\begin{aligned}
\sup _{G_{\varrho / 2}^{\varrho}}|\nabla u| \leq & c \varrho^{-1}\left\{|u|_{0, G_{\varrho / 4}^{2 \varrho}}+\|f\|_{\mathbf{V}_{q, 2 q-N}^{0}\left(G_{\varrho / 4}^{2 \varrho}\right)}\right. \\
& \left.+\|g\|_{\mathbf{V}_{q, 2 q-n}^{1-1 / q}\left(\Gamma_{\varrho / 4}^{2 \varrho}\right)}+\|h\|_{\mathbf{V}_{q, 2 q-n}^{1-1 / q}\left(\Sigma_{\varrho / 4}^{2 \varrho}\right.}\right\}
\end{aligned}
$$


and

$$
\begin{aligned}
\|u\|_{\mathbf{V}_{q, 2 q-n}^{2}\left(G_{\varrho / 2}^{\varrho}\right)} \leq & c\left\{|u|_{0, G_{\varrho / 4}^{2 \varrho}}+\|f\|_{\mathbf{V}_{q, 2 q-N}^{0}\left(G_{\varrho / 4}^{2 \varrho}\right)}\right. \\
& \left.+\|g\|_{\mathbf{V}_{q, 2 q-n}^{1-1 / q}\left(\Gamma_{\varrho / 4}^{2 \varrho}\right)}+\|h\|_{\mathbf{V}_{q, 2 q-n}^{1-1 / q}\left(\Sigma_{\varrho / 4}^{2 \varrho}\right)}\right\} .
\end{aligned}
$$

Hence, because of (1.4), (1.5) and assumption (c), the required results (1.6) and (1.7) follow.

Now we can make the statement of Theorem 5.2 more precise. In fact, the estimate (1.4) proved above allows us to consider in Theorem 5.2 the value $\varkappa=\lambda-1$. As a result we obtain the last statement of our theorem and the estimate (1.9) with the best possible exponent that satisfies the inequality (1.8).

\section{Examples: Eigenvalue transmission problem in a composite} plane domain with an angular point. We study the transmission boundary value problem $(L)$. Let $G \subset \mathbb{R}^{2}$ be a bounded domain with boundary $\partial G$ that is a smooth curve everywhere except at the origin $\mathcal{O} \in \partial G$. Near $\mathcal{O}$ it is a fan that consists of $N$ corners with vertices at $\mathcal{O}$. Thus

$$
G=\bigcup_{i=1}^{N} G_{i} ; \quad \partial G=\bigcup_{j=0}^{N+1} \Gamma_{j} ; \quad \Sigma=\bigcup_{k=1}^{N-1} \Sigma_{k} .
$$

Here $\Sigma_{k}, k=1, \ldots, N-1$, are rays that divide $G$ into angular domains $G_{i}$, $i=1, \ldots, N$. Let $\omega_{i}$ be the aperture at the vertex $\mathcal{O}$ in $G_{i}, i=1, \ldots, N$. Define $\theta_{k}=\omega_{1}+\cdots+\omega_{k}$. If $(r, \omega)$ are the polar coordinates of $(x, y)$, then $\omega=0$ is the equation of the ray $\Gamma_{0}$ and $\omega=\theta_{N}$ is the equation of the ray $\Gamma_{N+1}$. Let $\Gamma=\bigcup_{j=1}^{N} \Gamma_{j}$ be the curvilinear portion of $\partial G$.

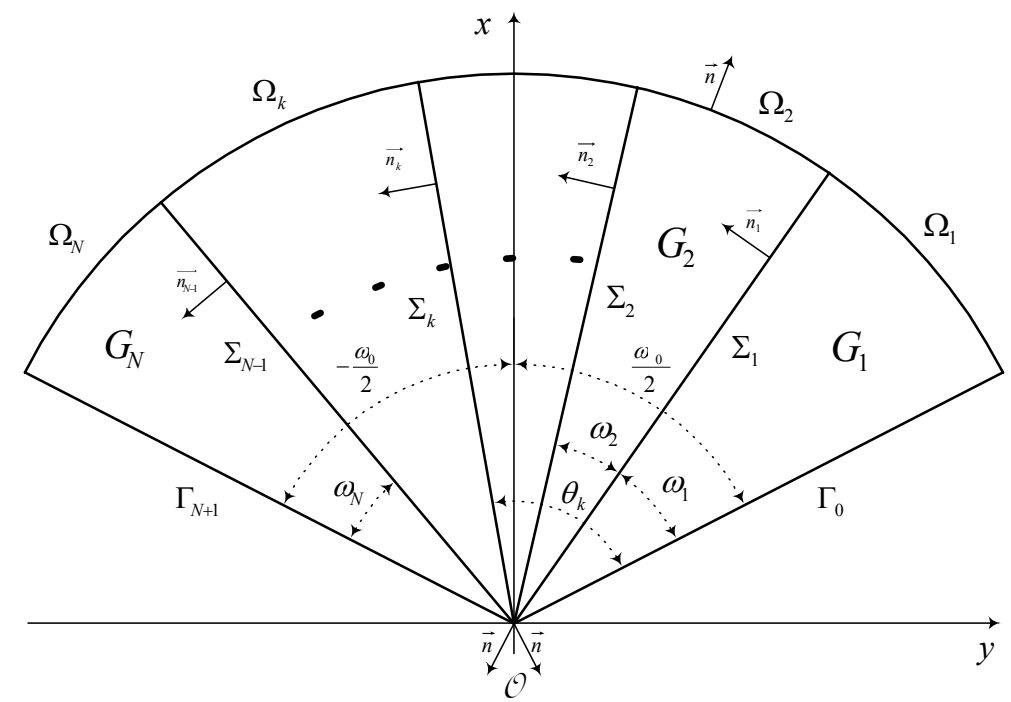

Fig. 2 
8.1. Three-media transmission problem. Our goal is the derivation of the eigenvalue equation that corresponds to our transmission problem for $N=3$. Let $S^{1}$ be the unit circle in $\mathbb{R}^{2}$ centered at $\mathcal{O}$. We define $\Omega_{i}=G_{i} \cap S^{1}$, $i=1,2,3$. The eigenvalue problem is the following (see also $[11,12]$ ):

$$
\left\{\begin{array}{l}
\psi_{i}^{\prime \prime}+\lambda^{2} \psi_{i}(\omega)=0, \quad \omega \in \Omega_{i}(i=1,2,3) \\
\psi_{1}\left(\omega_{1}\right)=\psi_{2}\left(\omega_{1}\right) ; \quad \psi_{3}\left(\theta_{2}\right)=\psi_{2}\left(\theta_{2}\right) \\
a_{2} \psi_{2}^{\prime}\left(\omega_{1}\right)-a_{1} \psi_{1}^{\prime}\left(\omega_{1}\right)+\beta_{1} \psi_{1}\left(\omega_{1}\right)=0 \\
a_{3} \psi_{3}^{\prime}\left(\theta_{2}\right)-a_{2} \psi_{2}^{\prime}\left(\theta_{2}\right)+\beta_{2} \psi_{2}\left(\theta_{2}\right)=0 \\
\alpha_{0} \psi_{1}^{\prime}(0)-\gamma_{0} \psi_{1}(0)=0 \\
\alpha_{3} \psi_{3}^{\prime}\left(\theta_{3}\right)-\gamma_{3} \psi_{3}\left(\theta_{3}\right)=0
\end{array}\right.
$$

where $\alpha_{0}=\left.\alpha\right|_{\Gamma_{0}}=\left.\alpha\right|_{\omega=0}, \alpha_{3}=\left.\alpha\right|_{\Gamma_{4}}=\left.\alpha\right|_{\omega=\theta_{3}}, \gamma_{0}=\gamma(0), \gamma_{3}=\gamma\left(\theta_{3}\right)$.

We find the general solution of (8.1):

$$
\begin{aligned}
& \psi_{i}(\omega)=A_{i} \cos (\lambda \omega)+B_{i} \sin (\lambda \omega), \\
& \psi_{i}^{\prime}(\omega)=-\lambda A_{i} \sin (\lambda \omega)+\lambda B_{i} \cos (\lambda \omega) \quad(i=1,2,3),
\end{aligned}
$$

where $A_{i}, B_{i}(i=1,2,3)$ are any constants. From the boundary condition of (8.1) we obtain a homogeneous algebraic system of six linear equations for $A_{i}, B_{i}(i=1,2,3)$. The determinant of the system must be zero for a nontrivial solution to exist. This gives the required eigenvalue equation:

$$
\begin{gathered}
\text { (8.2) }\left[\lambda^{2} \alpha_{3} a_{3}\left(\beta_{1} \gamma_{0}+\lambda^{2} \alpha_{0} a_{1}\right)-\gamma_{3}\left(\beta_{1} \beta_{2} \gamma_{0}+\lambda^{2} \alpha_{0} \beta_{2} a_{1}-\lambda^{2} \gamma_{0} a_{2}^{2}\right)\right] \\
\cdot \sin \left(\lambda \omega_{1}\right) \sin \left(\lambda \omega_{2}\right) \sin \left(\lambda \omega_{3}\right) \\
+\lambda \cdot\left[\lambda^{2} \alpha_{3} a_{3}\left(\beta_{1} \alpha_{0}-\gamma_{0} a_{1}\right)-\gamma_{3}\left(\beta_{1} \beta_{2} \alpha_{0}-\gamma_{0} \beta_{2} a_{1}-\lambda^{2} \alpha_{0} a_{2}^{2}\right)\right]
\end{gathered}
$$$$
\cdot \cos \left(\lambda \omega_{1}\right) \sin \left(\lambda \omega_{2}\right) \sin \left(\lambda \omega_{3}\right)
$$$$
+\lambda \cdot\left[\gamma_{3} a_{3}\left(\beta_{1} \gamma_{0}+\lambda^{2} \alpha_{0} a_{1}\right)+\alpha_{3}\left(\beta_{1} \beta_{2} \gamma_{0}+\lambda^{2} \alpha_{0} \beta_{2} a_{1}-\lambda^{2} \gamma_{0} a_{2}^{2}\right)\right]
$$$$
\cdot \sin \left(\lambda \omega_{1}\right) \sin \left(\lambda \omega_{2}\right) \cos \left(\lambda \omega_{3}\right)
$$

$$
\begin{aligned}
+\lambda^{2} \cdot\left[\gamma_{3} a_{3}\left(\beta_{1} \alpha_{0}-\gamma_{0} a_{1}\right)+\alpha_{3}\left(\beta_{1} \beta_{2} \alpha_{0}-\gamma_{0} \beta_{2} a_{1}-\lambda^{2} \alpha_{0} a_{2}^{2}\right)\right] \\
\cdot \cos \left(\lambda \omega_{1}\right) \sin \left(\lambda \omega_{2}\right) \cos \left(\lambda \omega_{3}\right)
\end{aligned}
$$

$+\lambda a_{2} \cdot\left[\gamma_{3}\left(\beta_{2} \gamma_{0}+\lambda^{2} \alpha_{0} a_{1}+\beta_{1} \gamma_{0}\right)-\lambda^{2} \alpha_{3} \gamma_{0} a_{3}\right] \cdot \sin \left(\lambda \omega_{1}\right) \cos \left(\lambda \omega_{2}\right) \sin \left(\lambda \omega_{3}\right)$

$+\lambda^{2} a_{2} \cdot\left[\gamma_{3}\left(\beta_{2} \alpha_{0}+\alpha_{0} \beta_{1}-\gamma_{0} a_{1}\right)-\lambda^{2} \alpha_{3} \alpha_{0} a_{3}\right] \cdot \cos \left(\lambda \omega_{1}\right) \cos \left(\lambda \omega_{2}\right) \sin \left(\lambda \omega_{3}\right)$

$-\lambda^{2} a_{2} \cdot\left[\gamma_{0} \gamma_{3} a_{3}+\alpha_{3}\left(\beta_{2} \gamma_{0}+\lambda^{2} \alpha_{0} a_{1}+\beta_{1} \gamma_{0}\right)\right] \cdot \sin \left(\lambda \omega_{1}\right) \cos \left(\lambda \omega_{2}\right) \cos \left(\lambda \omega_{3}\right)$

$-\lambda^{3} a_{2} \cdot\left[\alpha_{0} \gamma_{3} a_{3}+\alpha_{3}\left(\beta_{2} \alpha_{0}+\alpha_{0} \beta_{1}-a_{1} \gamma_{0}\right)\right] \cdot \cos \left(\lambda \omega_{1}\right) \cos \left(\lambda \omega_{2}\right) \cos \left(\lambda \omega_{3}\right)=0$.

We consider the special cases of boundary conditions.

- Dirichlet problem: $\alpha_{0}=\alpha_{3}=\beta_{1}=\beta_{2}=0 ; \gamma_{0}=\gamma_{3}=1$. Then $a_{2}^{2} \cdot \sin \left(\lambda \omega_{1}\right) \sin \left(\lambda \omega_{2}\right) \sin \left(\lambda \omega_{3}\right)-a_{1} a_{3} \cdot \cos \left(\lambda \omega_{1}\right) \sin \left(\lambda \omega_{2}\right) \cos \left(\lambda \omega_{3}\right)$ $-a_{1} a_{2} \cdot \cos \left(\lambda \omega_{1}\right) \cos \left(\lambda \omega_{2}\right) \sin \left(\lambda \omega_{3}\right)-a_{2} a_{3} \cdot \sin \left(\lambda \omega_{1}\right) \cos \left(\lambda \omega_{2}\right) \cos \left(\lambda \omega_{3}\right)=0$. 
In the isotropic case $\left(a_{1}=a_{2}=a_{3}\right)$ we hence obtain the well known result:

$$
\sin \left(\lambda \theta_{3}\right)=0, \quad \text { so } \quad \lambda_{n}=\frac{\pi n}{\theta_{3}}, \quad n=1,2, \ldots
$$

Corollary: $\lambda=\pi / \theta_{3}>1$ if $\omega_{1}+\omega_{2}+\omega_{3}<\pi$.

- Neumann problem: $\alpha_{0}=\alpha_{3}=1 ; \beta_{1}=\beta_{2}=\gamma_{0}=\gamma_{3}=0$. Then

$a_{2}^{2} \cdot \cos \left(\lambda \omega_{1}\right) \sin \left(\lambda \omega_{2}\right) \cos \left(\lambda \omega_{3}\right)+a_{2} a_{3} \cdot \cos \left(\lambda \omega_{1}\right) \cos \left(\lambda \omega_{2}\right) \sin \left(\lambda \omega_{3}\right)$

$+a_{1} a_{2} \cdot \sin \left(\lambda \omega_{1}\right) \cos \left(\lambda \omega_{2}\right) \cos \left(\lambda \omega_{3}\right)-a_{1} a_{3} \cdot \sin \left(\lambda \omega_{1}\right) \sin \left(\lambda \omega_{2}\right) \sin \left(\lambda \omega_{3}\right)=0$.

In the isotropic case $\left(a_{1}=a_{2}=a_{3}\right)$ we hence obtain the well known result:

$$
\sin \left(\lambda \theta_{3}\right)=0, \quad \text { so } \quad \lambda_{n}=\frac{\pi n}{\theta_{3}}, \quad n=0,1,2, \ldots
$$

Corollary: $\lambda=\pi / \theta_{3}>1$ if $\omega_{1}+\omega_{2}+\omega_{3}<\pi$.

- Mixed problem: $\alpha_{0}=\gamma_{3}=1, \alpha_{3}=\beta_{1}=\beta_{2}=\gamma_{0}=0$. Then

$$
\begin{gathered}
a_{2}^{2} \cdot \cos \left(\lambda \omega_{1}\right) \sin \left(\lambda \omega_{2}\right) \sin \left(\lambda \omega_{3}\right)+a_{1} a_{3} \cdot \sin \left(\lambda \omega_{1}\right) \sin \left(\lambda \omega_{2}\right) \cos \left(\lambda \omega_{3}\right) \\
+a_{1} a_{2} \cdot \sin \left(\lambda \omega_{1}\right) \cos \left(\lambda \omega_{2}\right) \sin \left(\lambda \omega_{3}\right)-a_{2} a_{3} \cdot \cos \left(\lambda \omega_{1}\right) \cos \left(\lambda \omega_{2}\right) \cos \left(\lambda \omega_{3}\right)=0 .
\end{gathered}
$$

In the isotropic case $\left(a_{1}=a_{2}=a_{3}\right)$ we hence obtain the well known result:

$$
\cos \left(\lambda \theta_{3}\right)=0, \quad \text { so } \quad \lambda_{n}=\frac{\pi(2 n-1)}{2 \theta_{3}}, \quad n=1,2, \ldots
$$

Corollary: $\lambda=\pi / 2 \theta_{3}>1$ if $\omega_{1}+\omega_{2}+\omega_{3}<\pi / 2$.

- Robin problem: $\alpha_{0}=1, \alpha_{3}=-1 ; \beta_{1}=\beta_{2}=0$.

$$
\begin{gathered}
\quad\left(\lambda^{2} a_{1} a_{3}-\gamma_{0} \gamma_{3} a_{2}^{2}\right) \cdot \sin \left(\lambda \omega_{1}\right) \sin \left(\lambda \omega_{2}\right) \sin \left(\lambda \omega_{3}\right) \\
-\lambda \cdot\left(\gamma_{3} a_{2}^{2}+\gamma_{0} a_{1} a_{3}\right) \cdot \cos \left(\lambda \omega_{1}\right) \sin \left(\lambda \omega_{2}\right) \sin \left(\lambda \omega_{3}\right) \\
-\lambda \cdot\left(\gamma_{3} a_{1} a_{3}+\gamma_{0} a_{2}^{2}\right) \cdot \sin \left(\lambda \omega_{1}\right) \sin \left(\lambda \omega_{2}\right) \cos \left(\lambda \omega_{3}\right) \\
+\left(\gamma_{0} \gamma_{3} a_{1} a_{3}-\lambda^{2} a_{2}^{2}\right) \cdot \cos \left(\lambda \omega_{1}\right) \sin \left(\lambda \omega_{2}\right) \cos \left(\lambda \omega_{3}\right) \\
-\lambda a_{2} \cdot\left(\gamma_{3} a_{1}+\gamma_{0} a_{3}\right) \cdot \sin \left(\lambda \omega_{1}\right) \cos \left(\lambda \omega_{2}\right) \sin \left(\lambda \omega_{3}\right) \\
+a_{2} \cdot\left(\gamma_{3} \gamma_{0} a_{1}-\lambda^{2} a_{3}\right) \cdot \cos \left(\lambda \omega_{1}\right) \cos \left(\lambda \omega_{2}\right) \sin \left(\lambda \omega_{3}\right) \\
+a_{2} \cdot\left(\gamma_{0} \gamma_{3} a_{3}-\lambda^{2} a_{1}\right) \cdot \sin \left(\lambda \omega_{1}\right) \cos \left(\lambda \omega_{2}\right) \cos \left(\lambda \omega_{3}\right) \\
+\lambda a_{2} \cdot\left(\gamma_{3} a_{3}+a_{1} \gamma_{0}\right) \cdot \cos \left(\lambda \omega_{1}\right) \cos \left(\lambda \omega_{2}\right) \cos \left(\lambda \omega_{3}\right)=0 .
\end{gathered}
$$

In the isotropic case $\left(a_{1}=a_{2}=a_{3}\right)$ we hence obtain the well known result (see [3, Example 1, §10.1.7])

$$
\tan \left(\lambda \theta_{3}\right)=\frac{\lambda\left(\gamma_{0}+\gamma_{3}\right)}{\lambda^{2}-\gamma_{0} \gamma_{3}}
$$

8.2. Two-media transmission problem. Setting in the above derived equations $\omega_{2}=0, \beta_{2}=0$ we get the corresponding results for domains with two different media $(N=2)$. The eigenvalue problem in this case has the form 


$$
\left\{\begin{array}{l}
\psi_{i}^{\prime \prime}+\lambda^{2} \psi_{i}(\omega)=0, \quad \omega \in \Omega_{i}(i=1,2) \\
\psi_{1}\left(\omega_{1}\right)=\psi_{2}\left(\omega_{1}\right) \\
a_{2} \psi_{2}^{\prime}\left(\omega_{1}\right)-a_{1} \psi_{1}^{\prime}\left(\omega_{1}\right)+\beta_{1} \psi_{1}\left(\omega_{1}\right)=0 \\
\alpha_{0} \psi_{1}^{\prime}(0)-\gamma_{0} \psi_{1}(0)=0 \\
\alpha_{2} \psi_{2}^{\prime}\left(\theta_{2}\right)-\gamma_{2} \psi_{2}\left(\theta_{2}\right)=0
\end{array}\right.
$$

The eigenvalue equation is

$$
\begin{aligned}
& {\left[\lambda^{2}\left(\alpha_{2} \gamma_{0} a_{2}-\alpha_{0} \gamma_{2} a_{1}\right)-\beta_{1} \gamma_{0} \gamma_{2}\right] \cdot \sin \left(\lambda \omega_{1}\right) \sin \left(\lambda \omega_{2}\right) } \\
+ & \lambda\left(\lambda^{2} \alpha_{0} \alpha_{2} a_{2}-\alpha_{0} \beta_{1} \gamma_{2}+\gamma_{0} \gamma_{2} a_{1}\right) \cdot \cos \left(\lambda \omega_{1}\right) \sin \left(\lambda \omega_{2}\right) \\
+ & \lambda\left(\lambda^{2} \alpha_{0} \alpha_{2} a_{1}+\lambda \beta_{1} \alpha_{2} \gamma_{0}+\gamma_{0} \gamma_{2} a_{2}\right) \cdot \sin \left(\lambda \omega_{1}\right) \cos \left(\lambda \omega_{2}\right) \\
+ & \lambda^{2}\left(\alpha_{0} \gamma_{2} a_{2}+\beta_{1} \alpha_{0} \alpha_{2}-\alpha_{2} \gamma_{0} a_{1}\right) \cdot \cos \left(\lambda \omega_{1}\right) \cos \left(\lambda \omega_{2}\right)=0 .
\end{aligned}
$$

Here we consider in detail the 2-dimensional transmission problem with two different media $\left(\omega_{1}=\omega_{2}=\omega_{0} / 2\right)$ for the Laplace operator in an angular symmetric domain and investigate the corresponding eigenvalue problem. Suppose $n=2$, the domain $G$ lies inside the corner

$$
G_{0}=\left\{(r, \omega) \mid r>0 ;-\omega_{0} / 2<\omega<\omega_{0} / 2\right\}, \quad \omega_{0} \in(0,2 \pi) ;
$$

$\mathcal{O} \in \partial G$ and in some neighborhood of $\mathcal{O}$ the boundary $\partial G$ coincides with the sides of the corner $\omega=-\omega_{0} / 2$ and $\omega=\omega_{0} / 2$. We define $\Gamma_{ \pm}=\{(r, \omega) \mid r>0$; $\left.\omega= \pm \omega_{0} / 2\right\}, \Sigma_{0}=\{(r, \omega) \mid r>0 ; \omega=0\}$ and put $\left.\sigma(\omega)\right|_{\Sigma_{0}}=\sigma(0)=\sigma=$ const $\geq 0,\left.\gamma(\omega)\right|_{\omega= \pm \omega_{0} / 2}=\gamma_{ \pm}=$const $>0$. We consider the following problem:

$$
\begin{cases}\Delta u_{ \pm}=f_{ \pm}(x), & x \in G_{ \pm} \\ {[u]_{\Sigma_{0}}=0, \quad\left[a \frac{\partial u}{\partial \vec{n}}\right]_{\Sigma_{0}}+\frac{1}{|x|} \sigma u(x)=h(x),} & x \in \Sigma_{0} \\ \alpha_{ \pm} \frac{\partial u_{ \pm}}{\partial \vec{n}}+\frac{1}{|x|} \gamma_{ \pm} u_{ \pm}(x)=g_{ \pm}(x), & x \in \Gamma_{ \pm} \backslash \mathcal{O}\end{cases}
$$

where

$$
\alpha_{ \pm}= \begin{cases}0 & \text { if } \Gamma_{ \pm} \in \mathcal{D} \\ \pm 1 & \text { if } \Gamma_{ \pm} \notin \mathcal{D}\end{cases}
$$

It is well known that the homogeneous problem $(f(x)=0, h(x)=0, g(x)=0)$ has a solution of the form $u(r, \omega)=r^{\lambda} \psi(\omega)$, where $\lambda$ is an eigenvalue and $\psi(\omega)$ is a regular eigenfunction associated to this $\lambda$ of the problem

$$
\left\{\begin{array}{l}
\psi_{+}^{\prime \prime}+\lambda^{2} \psi_{+}(\omega)=0, \quad \omega \in\left(0, \omega_{0} / 2\right) \\
\psi_{-}^{\prime \prime}+\lambda^{2} \psi_{-}(\omega)=0, \quad \omega \in\left(-\omega_{0} / 2,0\right) \\
\psi_{+}(0)=\psi_{-}(0) ; \quad a_{+} \psi_{+}^{\prime}(0)-a_{-} \psi_{-}^{\prime}(0)=\sigma \psi(0) \\
\pm \alpha_{ \pm} \psi^{\prime}\left( \pm \omega_{0} / 2\right)+\gamma_{ \pm} \psi\left( \pm \omega_{0} / 2\right)=0
\end{array}\right.
$$


1) Case $\lambda=0, \sigma>0$. In this case the solution of our equations has the form $\psi_{ \pm}(\omega)=A_{ \pm} \cdot \omega+B_{ \pm}$. From the boundary conditions we obtain $B_{+}=B_{-}=B$ and for $A_{+}, A_{-}, B$ we have the system

$$
\left\{\begin{array}{l}
a_{+} A_{+}-a_{-} A_{-}-\sigma B=0 \\
\left(\alpha_{+}+\frac{\omega_{0}}{2} \gamma_{+}\right) A_{+}+\gamma_{+} B=0 \\
-\left(\alpha_{-}+\frac{\omega_{0}}{2} \gamma_{-}\right) A_{-}+\gamma_{-} B=0
\end{array}\right.
$$

Since $A_{+}^{2}+A_{-}^{2}+B^{2} \neq 0$, the determinant of the system must be zero, that is,

$$
\begin{aligned}
\sigma\left(\alpha_{+}+\frac{\omega_{0}}{2} \gamma_{+}\right)\left(\alpha_{-}+\frac{\omega_{0}}{2} \gamma_{-}\right) & +a_{+} \gamma_{+}\left(\alpha_{-}+\frac{\omega_{0}}{2} \gamma_{-}\right) \\
& +a_{-} \gamma_{-}\left(\alpha_{+}+\frac{\omega_{0}}{2} \gamma_{+}\right)=0 .
\end{aligned}
$$

Thus if (8.7) holds, then $\lambda=0$ and the corresponding eigenfunction is

$$
\begin{aligned}
& \psi(\omega)= \\
& \begin{cases}-\gamma_{+}\left(\alpha_{-}+\frac{\omega_{0}}{2} \gamma_{-}\right)\left(\omega+\frac{a_{+}}{\sigma}\right)-\frac{a_{-} \gamma_{-}}{\sigma}\left(\alpha_{+}+\frac{\omega_{0}}{2} \gamma_{+}\right), & \omega \in\left(0, \omega_{0} / 2\right), \\
\gamma_{-}\left(\alpha_{+}+\frac{\omega_{0}}{2} \gamma_{+}\right)\left(\omega-\frac{a_{-}}{\sigma}\right)-\frac{a_{+} \gamma_{+}}{\sigma}\left(\alpha_{-}+\frac{\omega_{0}}{2} \gamma_{-}\right), & \omega \in\left(-\omega_{0} / 2,0\right) .\end{cases}
\end{aligned}
$$

2) Case $\lambda \neq 0$. In this case the solution of our equations has the form

$$
\psi_{ \pm}(\omega)=A_{ \pm} \cos (\lambda \omega)+B_{ \pm} \sin (\lambda \omega) .
$$

From the boundary conditions we obtain $A_{+}=A_{-}=A$ and for $A, B_{+}, B_{-}$ we have the system

$$
\left\{\begin{array}{l}
\sigma A-\lambda a_{+} B_{+}+\lambda a_{-} B_{-}=0, \\
\left(\gamma_{+} \cos \frac{\lambda \omega_{0}}{2}-\lambda \alpha_{+} \sin \frac{\lambda \omega_{0}}{2}\right) A+\left(\gamma_{+} \sin \frac{\lambda \omega_{0}}{2}+\lambda \alpha_{+} \cos \frac{\lambda \omega_{0}}{2}\right) B_{+}=0 \\
\left(\gamma_{-} \cos \frac{\lambda \omega_{0}}{2}-\lambda \alpha_{-} \sin \frac{\lambda \omega_{0}}{2}\right) A-\left(\gamma_{-} \sin \frac{\lambda \omega_{0}}{2}+\lambda \alpha_{-} \cos \frac{\lambda \omega_{0}}{2}\right) B_{-}=0 .
\end{array}\right.
$$

Since $A^{2}+B_{+}^{2}+B_{-}^{2} \neq 0$, the determinant must be zero; this means that $\lambda$ is defined from the transcendental equation

$$
\begin{aligned}
& \sigma\left(\lambda^{2} \alpha_{+} \alpha_{-}+\gamma_{+} \gamma_{-}\right)+\lambda^{2}\left(a_{+}-a_{-}\right)\left(\alpha_{-} \gamma_{+}-\alpha_{+} \gamma_{-}\right) \\
& +\lambda\left[\sigma\left(\alpha_{-} \gamma_{+}+\alpha_{+} \gamma_{-}\right)+\left(a_{+}+a_{-}\right)\left(\gamma_{+} \gamma_{-}-\lambda^{2} \alpha_{+} \alpha_{-}\right)\right] \sin \left(\lambda \omega_{0}\right) \\
& +\left[\sigma\left(\lambda^{2} \alpha_{+} \alpha_{-}-\gamma_{+} \gamma_{-}\right)+\lambda^{2}\left(a_{+}+a_{-}\right)\left(\alpha_{-} \gamma_{+}+\alpha_{+} \gamma_{-}\right)\right] \cos \left(\lambda \omega_{0}\right)=0 .
\end{aligned}
$$

Now we investigate each type of boundary conditions separately. 
The Dirichlet problem: $\alpha \pm=0$. The equation (8.8) takes the form $\sigma(1-$ $\left.\cos \left(\lambda \omega_{0}\right)\right)+\lambda\left(a_{+}+a_{-}\right) \sin \left(\lambda \omega_{0}\right)=0$. Hence

$$
\lambda= \begin{cases}\frac{\pi}{\omega_{0}} & \text { if } \sigma=0, \\ \text { the least positive root of } \tan \frac{\lambda \omega_{0}}{2}=-\frac{a_{+}+a_{-}}{\sigma} \cdot \lambda & \text { if } \sigma \neq 0,\end{cases}
$$

and the corresponding eigenfunction is

$$
\psi(\omega)= \begin{cases}\sin \lambda\left(\omega_{0} / 2-\omega\right), & \omega \in\left(0, \omega_{0} / 2\right) \\ \sin \lambda\left(\omega_{0} / 2+\omega\right), & \omega \in\left(-\omega_{0} / 2,0\right) .\end{cases}
$$

The Neumann problem: $\gamma \pm=0$. The equation (8.8) takes the form $\sigma(1+$ $\left.\cos \left(\lambda \omega_{0}\right)\right)-\lambda\left(a_{+}+a_{-}\right) \sin \left(\lambda \omega_{0}\right)=0$. Hence $\lambda=\min \left\{\lambda^{*}, \pi / \omega_{0}\right\}$, where $\lambda^{*}$ is the least positive root of the transcendental equation

$$
\tan \frac{\lambda \omega_{0}}{2}=\frac{\sigma}{a_{+}+a_{-}} \cdot \frac{1}{\lambda}
$$

For $\lambda=\pi / \omega_{0}$ we find the corresponding eigenfunction

$$
\psi(\omega)= \begin{cases}a_{-} \sin \left(\pi \omega / \omega_{0}\right), & \omega \in\left(0, \omega_{0} / 2\right), \\ a_{+} \sin \left(\pi \omega / \omega_{0}\right), & \omega \in\left(-\omega_{0} / 2,0\right) .\end{cases}
$$

For $\lambda=\lambda^{*}$ we find the corresponding eigenfunction

$$
\psi(\omega)= \begin{cases}\cos \lambda^{*}\left(\omega-\omega_{0} / 2\right), & \omega \in\left(0, \omega_{0} / 2\right), \\ \cos \lambda^{*}\left(\omega+\omega_{0} / 2\right), & \omega \in\left(-\omega_{0} / 2,0\right) .\end{cases}
$$

Mixed problem: $\alpha_{+}=1, \alpha_{-}=0 ; \gamma_{+}=0, \gamma_{-}=1$. The equation (8.8) takes the form

$$
\sigma \sin \left(\lambda \omega_{0}\right)+\lambda\left(a_{+}+a_{-}\right) \cos \left(\lambda \omega_{0}\right)=\lambda\left(a_{+}-a_{-}\right) .
$$

In particular, if $\sigma=0$, and $a_{+} a_{-}>0$ then

$$
\lambda=\frac{2}{\omega_{0}} \arctan \sqrt{a_{-} / a_{+}}>1, \quad \text { if } \omega_{0}<2 \arctan \sqrt{a_{-} / a_{+}},
$$

and the corresponding eigenfunction is

$$
\psi(\omega)= \begin{cases}\cos (\lambda \omega)+\sqrt{a_{-} / a_{+}} \cdot \sin (\lambda \omega), & \omega \in\left(0, \omega_{0} / 2\right), \\ \cos (\lambda \omega)+\sqrt{a_{+} / a_{-}} \cdot \sin (\lambda \omega), & \omega \in\left(-\omega_{0} / 2,0\right) .\end{cases}
$$

If $\lambda$ is the least positive root of the transcendental equation (8.9), then the corresponding eigenfunction is

$$
\psi(\omega)= \begin{cases}\sin \left(\lambda \omega_{0} / 2\right) \cos \lambda\left(\omega-\omega_{0} / 2\right), & \omega \in\left(0, \omega_{0} / 2\right), \\ \cos \left(\lambda \omega_{0} / 2\right) \sin \lambda\left(\omega+\omega_{0} / 2\right), & \omega \in\left(-\omega_{0} / 2,0\right) .\end{cases}
$$


The Robin problem: $\alpha_{ \pm}=a_{ \pm} ; \gamma_{ \pm} \neq 0$. The equation (8.8) takes the form $\begin{aligned} \sigma\left(\lambda^{2} a_{+} a_{-}\right. & \left.+\gamma_{+} \gamma_{-}\right)+\lambda^{2}\left(a_{+}-a_{-}\right)\left(a_{-} \gamma_{+}-a_{+} \gamma_{-}\right) \\ & +\lambda\left[\sigma\left(a_{-} \gamma_{+}+a_{+} \gamma_{-}\right)+\left(a_{+}+a_{-}\right)\left(\gamma_{+} \gamma_{-}-\lambda^{2} a_{+} a_{-}\right)\right] \sin \left(\lambda \omega_{0}\right) \\ & +\left[\sigma\left(\lambda^{2} a_{+} a_{-}-\gamma_{+} \gamma_{-}\right)+\lambda^{2}\left(a_{+}+a_{-}\right)\left(a_{-} \gamma_{+}+a_{+} \gamma_{-}\right)\right] \cos \left(\lambda \omega_{0}\right)=0 .\end{aligned}$

In particular, in the case of the problem without the interface $\left(a_{+}=a_{-}=1\right.$, $\sigma=0$ ) we obtain the least eigenvalue as the least positive root of the transcendental equation

$$
\tan \left(\lambda \omega_{0}\right)=\frac{\lambda\left(\gamma_{+}+\gamma_{-}\right)}{\lambda^{2}-\gamma_{+} \gamma_{-}}
$$

and the corresponding eigenfunction is

$$
\psi(\omega)=\lambda \cos \left[\lambda\left(\omega-\omega_{0} / 2\right)\right]-\gamma_{+} \sin \left[\lambda\left(\omega-\omega_{0} / 2\right)\right]
$$

(see $[3, \S 10.1 .7])$.

In order to have $\lambda>1$ we show that the condition $\gamma_{ \pm} \geq \gamma_{0}>\tan \left(\omega_{0} / 2\right)$ from the assumption (b) of our theorems is justified. In fact, we rewrite the equation (8.10) in the equivalent form

$$
\lambda=\frac{1}{\omega_{0}}\left(\arctan \frac{\gamma_{+}}{\lambda}+\arctan \frac{\gamma_{-}}{\lambda}\right) .
$$

Hence

$$
1<\lambda<\frac{1}{\omega_{0}}\left(\arctan \gamma_{+}+\arctan \gamma_{-}\right)
$$

so

$$
\omega_{0}<\arctan \frac{\gamma_{+}+\gamma_{-}}{1-\gamma_{+} \gamma_{-}} \quad \text { provided that } \gamma_{+} \gamma_{-}<1 .
$$

But assumption (b) means that $\gamma_{ \pm} \geq \gamma_{0}>\tan \left(\omega_{0} / 2\right)$. Hence

$$
\frac{\gamma_{+}+\gamma_{-}}{1-\gamma_{+} \gamma_{-}} \geq \frac{2 \gamma_{0}}{1-\gamma_{0}^{2}}>\frac{2 \tan \frac{\omega_{0}}{2}}{1-\tan ^{2} \frac{\omega_{0}}{2}}=\tan \omega_{0}, \quad \omega_{0}<\frac{\pi}{2} .
$$

Thus we get (8.12). In the case $\gamma_{ \pm} \geq \gamma_{0}>\tan \left(\omega_{0} / 2\right) \geq 1$ for $\omega_{0} \in[\pi / 2, \pi)$ the inequality $\lambda>1$ is fulfilled a fortiori, since the eigenvalues increase together with $\gamma(\phi)$ (see for example [6, Theorem 6, §2, Chapter VI]). In fact, $\lambda=1$ is the solution of the equation (8.10) under $\gamma_{ \pm}=\tan \left(\omega_{0} / 2\right)$.

8.3. The function

$$
u(r, \omega)=r^{\lambda} \ln \left(\frac{1}{r}\right) \psi(\omega)
$$

with $\lambda$ and $\psi(\omega)$ defined by (8.6) is a solution of the problem (8.5) with $f_{ \pm}=-2 a_{ \pm} \lambda r^{\lambda-2} \psi(\omega), h(x)=0, g(x)=0$ in the corner $G_{0}$. All assumptions of Theorem 1.1 are satisfed with $s=\lambda$. This example shows the precision of the assumptions on the right sides of $(L)$ in Theorem 1.1. 


\section{References}

[1] S. Agmon, A. Douglis and L. Nirenberg, Estimates near the boundary for solutions of elliptic partial differential equations satisfying general boundary conditions, Comm. Pure Appl. Math. 12 (1959), 623-727.

[2] M. V. Borsuk, A priori estimates and solvability of second order quasilinear elliptic equations in a composite domain with nonlinear boundary conditions and conjunction condition, Proc. Steklov Inst. Math. 103 (1970), 13-51.

[3] M. Borsuk and V. Kondratiev, Elliptic Boundary Value Problems of Second Order in Piecewise Smooth Domains, North-Holland Math. Library 69, Elsevier, 2006.

[4] Y.-Z. Chen and L.-C. Wu, Second Order Elliptic Equations and Elliptic Systems, Transl. Math. Monogr. 174, Amer. Math. Soc., Providence, RI, 1998.

[5] W. Chikouche, D. Mercier and S. Nicaise, Regularity of the solution of some unilateral boundary value problems in polygonal and polyhedral domains, Comm. Partial Differential Equations 29 (2004), 43-70.

[6] R. Courant und D. Hilbert, Methoden der mathematischen Physik, Bd. 1, Springer, Berlin, 1931.

[7] D. Gilbarg and N. S. Trudinger, Elliptic Partial Differential Equations of Second Order, Springer, Berlin, 1977; revised 3rd printing, 1998.

[8] G. H. Hardy, J. E. Littlewood and G. Pólya, Inequalities, Cambridge Univ. Press., 1952.

[9] V. A. Il'in, On the solvability of the Dirichlet and Neumann problems for linear elliptic operators with discontinuous coefficients, Dokl. Akad. Nauk SSSR 137 (1961), 28-30 (in Russian).

[10] O. A. Ladyzhenskaya and N. N. Ural'tseva, Linear and Quasilinear Elliptic Equations, Academic Press, New York, 1968.

[11] S. Nicaise, Polygonal Interface Problems, Peter Lang, 1993.

[12] S. Nicaise and A.-M. Sändig, General interface problems I, II, Math. Meth. Appl. Sci. 17 (1994), 395-450.

[13] Z. G. Sheftel, Estimates in $L_{p}$ of solutions of elliptic equations with discontinuous coefficients and satisfying general boundary conditions and conjugacy conditions, Soviet Math. Dokl. 4 (1963), 321-324.

Department of Mathematics and Informatics

University of Warmia and Mazury in Olsztyn

10-957 Olsztyn-Kortowo, Poland

E-mail: borsuk@uwm.edu.pl 
